\title{
Inexistência de difusão sublinear para uma classe de homeomorfismos do toro
}

\author{
Guilherme Silva Salomão
}

TESE APRESENTADA

$\mathrm{AO}$

Instituto DE MatemáticA E EstatísticA

DA

Universidade DE SÃo PAUlo

PARA

OBTENÇÃO DO TÍTULO

$\mathrm{DE}$

DOUTOR EM CIÊNCIAS

Programa: Matemática Aplicada

Orientador: Prof. Dr. Fabio Armando Tal

Durante o desenvolvimento deste trabalho o autor recebeu auxílio financeiro da FAPESP

São Paulo, março de 2019 


\title{
Inexistência de difusão sublinear para uma classe de homeomorfismos do toro
}

\author{
Esta versão da tese contém as correções e alterações sugeridas \\ pela Comissão Julgadora durante a defesa da versão original do trabalho, \\ realizada em 30/01/2019. Uma cópia da versão original está disponível no \\ Instituto de Matemática e Estatística da Universidade de São Paulo.
}

Comissão Julgadora:

- Prof. Dr. Fabio Armando Tal (orientador) - IME-USP

- Prof. Dr. Salvador Addas Zanata - IME-USP

- Prof. Dr. Andrés Koropecki - UFF

- Prof. Dr. Alejandro Kocsard - UFF

- Prof. Dr. Alejandro Miguel Passeggi Diaz Robles - FEAUDELAR 


\section{Agradecimentos}

Agradeço primeiramente a Deus, por tudo. Agradeço também à Jéssica, por todo o apoio e amor, meus pais e minha família por todo o amor e incentivo.

Quero agradecer também meu orientador, Prof. Dr. Fabio Armando Tal, por me acompanhar e ensinar ao longo desses anos. Agradeço também aos colegas de IME: Marco, Lucas e 79 pela amizade e companheirismo nessa jornada.

Agradeço também à FAPESP pelo apoio financeiro do presente trabalho. 


\section{Resumo}

\section{SALOMÃO, G. S. Inexistência de difusão sublinear para uma classe de homeomorfismos}

do toro. 2018. 120 f. Tese (Doutorado) - Instituto de Matemática e Estatística, Universidade de São Paulo, São Paulo, 2018.

No presente trabalho iremos provar, usando a folheação de Brouwer-Le Calvez e a teoria de forcing dela derivada, que dado um homeomorfismo do toro $f: \mathbb{T}^{2} \rightarrow \mathbb{T}^{2}$ isotópico à identidade tal que seu conjunto de rotação $\rho(\tilde{f})=\left\{t \rho_{0} \mid 0 \leq t \leq 1\right\}$ é um segmento de reta com inclinação irracional e tendo 0 como um ponto extremal, então existe $M>0$ tal que $\left|\left\langle\tilde{f}^{n}(\tilde{x})-\tilde{x}, \rho_{0}^{\perp}\right\rangle\right|<M$, para todo $\tilde{x} \in \mathbb{R}^{2}$ e $n \in \mathbb{Z}$, ou em outras palavras, $f$ não possui difusão sublinear na direção perpendicular à direção do conjunto de rotação

Palavras-chave: homeomorfismos do toro, dinâmica topológica, conjunto de rotação, difusão sublinear. 


\section{Abstract}

SALOMÃO, G. S. Inexistence of sublinear diffusion for a class of torus homeomorphisms. 2018. 120 f. Tese (Doutorado) - Instituto de Matemática e Estatística, Universidade de São Paulo, São Paulo, 2018.

In the present work we will prove, using the Brouwer-Le Calvez foliation and the forcing theory derived from it, that given a torus homeomorphism $f: \mathbb{T}^{2} \rightarrow \mathbb{T}^{2}$ isotopopic to the identity such that its rotation set $\rho(\tilde{f})=\left\{t \rho_{0} \mid 0 \leq t \leq 1\right\}$ is a line segment with irrational slope and 0 is an extreme point, then there exists $M>0$ such that $\left|\left\langle\tilde{f}^{n}(\tilde{x})-\tilde{x}, \rho_{0}^{\perp}\right\rangle\right|<M$, for every $\tilde{x} \in \mathbb{R}^{2}$ and $n \in \mathbb{Z}$, or in other words, $f$ does not have sublinear diffusion in the direction perpendicular to the direction of the rotation set.

Keywords: torus homeomorphisms, topological dynamics, rotation set, sublinear diffusion. 


\section{Sumário}

$\begin{array}{ll}\text { Lista de Figuras } & \text { ix }\end{array}$

1 Introdução e estrutura $\quad 1$

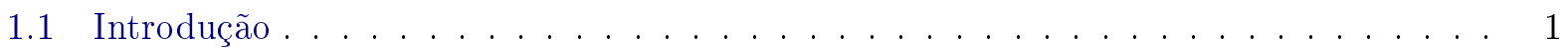

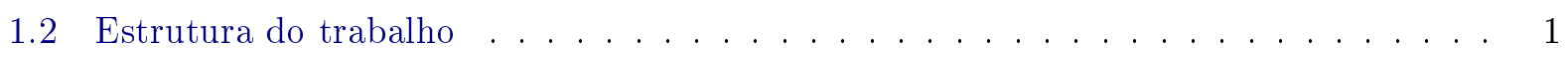

2 Teoria de Rotação $\quad 3$

2.1 Homeomorfismos do círculo . . . . . . . . . . . . . . . . . . 3

2.2 Conjunto de rotação para homeomorfismos do toro . . . . . . . . . . . . . 3

2.3 Difusão Sublinear . . . . . . . . . . . . . . . . . . . . . . 6

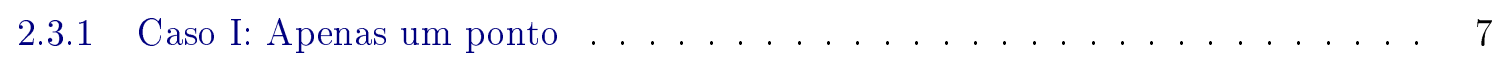

2.3 .2 Caso II: Interior não vazio . . . . . . . . . . . . . . . . . . 8

2.3 .3 Caso III: Segmento de reta $\ldots \ldots \ldots \ldots \ldots$

2.3 .4 Dehn twists . . . . . . . . . . . . . . . . . . . 9

2.4 Dinâmica Essencial . . . . . . . . . . . . . . . . . . . . . . 9

3 Folheação de Brouwer-Le Calvez e Forcing $\quad 13$

3.1 Homeomorfismos de Brouwer . . . . . . . . . . . . . . . . . . . . 13

3.2 Folheação de Brouwer-Le Calvez . . . . . . . . . . . . . . . . . . . 14

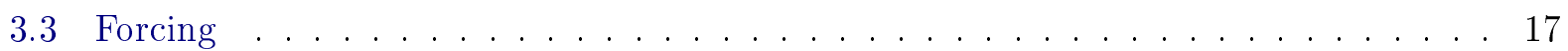

4 Difusão sublinear $\quad 21$

4.1 Enunciado do problema . . . . . . . . . . . . . . . . . . . 21

4.2 Demonstração do Teorema A . . . . . . . . . . . . . . . . 21

$\begin{array}{ll}\text { Referências Bibliográficas } & 37\end{array}$ 
viii SUMÁRIO 


\section{Lista de Figuras}

3.1 Um caminho transverso $\gamma \ldots \ldots \ldots \ldots \ldots \ldots \ldots \ldots$

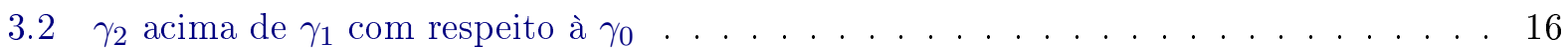

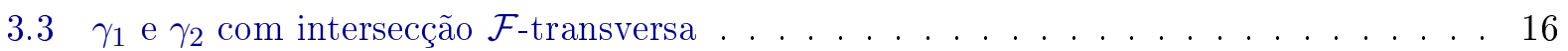

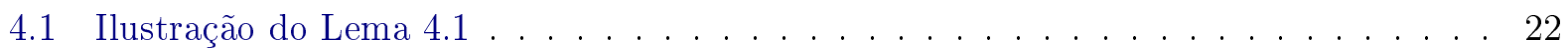

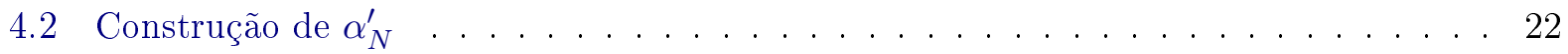

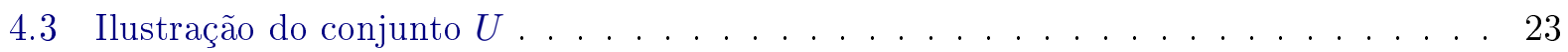

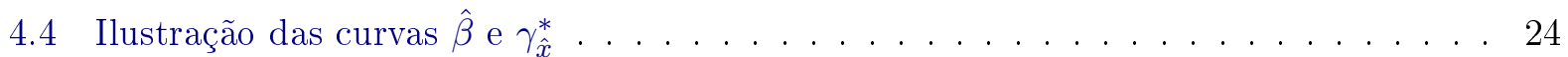

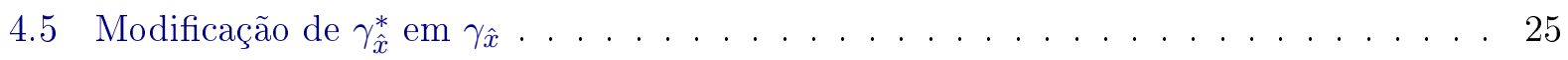

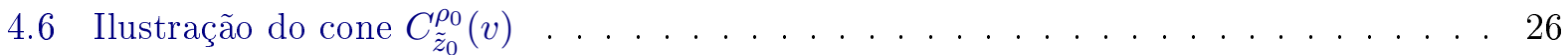

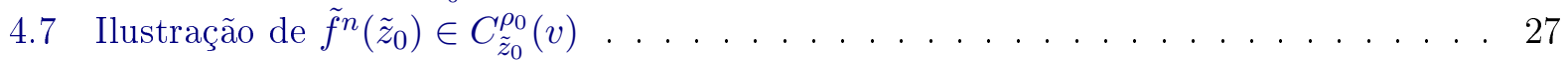

4.8 Demonstração do Lema $4.10 \ldots \ldots$. . . . . . . . . . . . . . . . 29

4.9 Ilustração do Lema $4.18 \ldots \ldots \ldots$. . . . . . . . . . . . . . . . 31

4.10 Ilustração do Lema $4.18 \ldots \ldots$. . . . . . . . . . . . . . . . . . 32

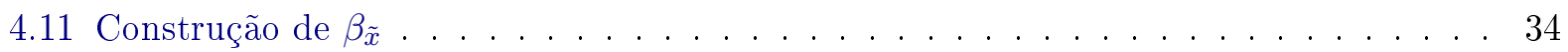

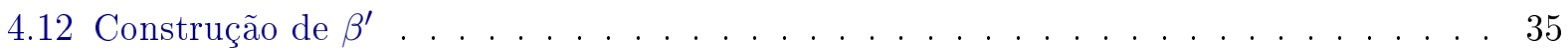

4.13 Intersecção de $\beta^{\prime} \operatorname{com} \beta^{\prime}+w_{3}-w_{1} \ldots \ldots \ldots \ldots \ldots \ldots$ 


\section{Capítulo 1}

\section{Introdução e estrutura}

\subsection{Introdução}

Desde sua introdução por Poincaré, o conceito de número ou conjunto de rotação tem sido de grande utilidade no estudo de dinâmica, seja de aplicações do círculo, seja de aplicações de superfícies. No presente trabalho nos concentraremos no conjunto de rotação para o toro bidimensinal. Introduzido na década de 1980 (veja [MZ89]), tem sido objeto de muitos estudos ao longo dos últimos anos, com muitos resultados sendo obtidos por diversos autores. De forma geral, podemos categorizar os resultados em dois tipos, o primeiro seria, quais conjuntos do plano podem ser realizados como conjunto de rotação, para alguma aplicação do toro? O outro tipo, que será o foco deste trabalho, seria, assumindo que o conjunto de rotação tem uma determinada forma, quais propriedades podemos deduzir sobre a dinâmica da aplicação em questão?

Intuitivamente, o conjunto de rotação informa as velocidades lineares com que uma aplicação do toro faz os pontos rodarem. Contudo, é válido perguntar se existe algum tipo de comportamento rotacional que não é capturado por tal conjunto. Existem muitos resultados que mostram que, sob determinadas hipóteses e para determinados tipos de conjunto de rotação, não existem pontos que se movem com velocidades sublineares. Contudo, existem exemplos que ilustram que esse nem sempre é o caso, é possível construir aplicações que possuem velocidades sublineares não capturadas pelo conjunto de rotação.

Nosso objetivo será mostrar que se um homeomorfismo do toro $f$ tem como conjunto de rotação um segmento de reta com inclinação irracional e tendo a origem como ponto extremal, então $f$ não possui movimentos rotacionais na direção perpendicular ao conjunto de rotação. Mais precisamente, provaremos o seguinte:

Teorema A. Suponhamos que $\rho(\tilde{f})=\left\{t \rho_{0} \mid 0 \leq t \leq 1\right\}$, onde $\tan \left(\rho_{0}\right) \notin \mathbb{Q}$. Então existe $M>0$ tal que

$$
\left|\left\langle\tilde{f}^{n}(\tilde{x})-\tilde{x}, \rho_{0}^{\perp}\right\rangle\right|<M,
$$

para todo $\tilde{x} \in \mathbb{R}^{2}$ e $n \in \mathbb{Z}$.

Note que o Teorema A apresenta a solução para o problema da difusão sublinear para um dos últimos casos restantes entre os tipos de conjunto de rotação que sabemos que são realizados por algum homeomorfismo. Existem conjuntos compactos e convexos para os quais ainda não foram obtidos resultados sobre difusão sublinear, contudo até o presente momento não foram encontrados resultados que confirmem que tais conjuntos podem ser realizados como conjuntos de rotação.

\subsection{Estrutura do trabalho}

No Capítulo 2 apresentaremos os resultados básicos sobre teoria de rotação, bem como uma breve coletânea de resultados similares ao teorema principal deste trabalho, mas para outras hipóteses sobre o conjunto de rotação. Também no Capítulo 2 apresentaremos um breve resumo sobre 
dinâmica essencial, além de alguns resultados que utilizaremos na demonstração do nosso teorema. O Capítulo 3 é dedicado ao estudo da principal ferramenta utilizada ao longo do texto, a Folheação de Brouwer-Le Calvez e a teoria de Forcing dela derivada. Apresentaremos as definições básicas, bem como os principais resultados e alguns teoremas auxiliares que serão utilizados na etapa final. Por fim, no Capítulo 4 apresentaremos a demonstração do Teorema A. 


\section{Capítulo 2}

\section{Teoria de Rotação}

\section{$2.1 \quad$ Homeomorfismos do círculo}

A teoria de rotação teve seu início com Henri Poincaré, [Poi81], onde foi definido o número de rotação para homeomorfismos do círculo da seguinte forma:

Definição 2.1. Seja $h: \mathbb{S}^{1} \rightarrow \mathbb{S}^{1}$ um homeomorfismo que preserva orientação, onde $\mathbb{S}^{1}=\mathbb{R} / \mathbb{Z}$. Definimos o número de rotação de $h$ como

$$
\rho(\tilde{h})=\lim _{n \rightarrow+\infty} \frac{\tilde{h}^{n}(\tilde{z})-\tilde{z}}{n},
$$

onde $\tilde{h}: \mathbb{R} \rightarrow \mathbb{R}$ é um levantamento de $h, \tilde{z} \in \pi^{-1}(z)$ para algum $z \in \mathbb{S}^{1}$ e $\pi: \mathbb{R} \rightarrow \mathbb{S}^{1}$ é a aplicação de recobrimeto.

É possível mostrar que tal limite existe e não depende da escolha do ponto $z$. Além disso, se $\tilde{h}^{\prime}$ é outro levantamento de $h$, então $\tilde{h}^{\prime}=\tilde{h}+k$, para algum $k \in \mathbb{Z}$, e portanto $\rho\left(\tilde{h}^{\prime}\right)=\rho(\tilde{h})+k$.

Dois resultados que resumem bem o poder do número de rotação na descrição da dinâmica de $h$ são os seguintes:

Proposição 2.2. Temos que $\rho(\tilde{h}) \in \mathbb{Q}$ se e somente se $h$ possui ponto periódico.

Teorema 2.3. Se $h: \mathbb{S}^{1} \rightarrow \mathbb{S}^{1}$ é um difeomorfismo de classe $\mathcal{C}^{1}$ que preserva orientação, com derivada de variação limitada e número de rotação $\rho(\tilde{h}) \in \mathbb{R} \backslash \mathbb{Q}$, então h é topologicamente conjugado à $\operatorname{rotação~} x \mapsto x+\rho(\tilde{h})(\bmod 1)$.

Veja [KH95] para as demonstrações, além de outros resultados que usam o número de rotação para extrair informações sobre a dinâmica de $h$ e classificar os homeomorfimos segundo características de seus números de rotação. Com isso em mente, é natural tentar estender tal conceito para outros espaços.

\subsection{Conjunto de rotação para homeomorfismos do toro}

Vejamos agora como definir o conjunto de rotação para homeomorfismos do toro $\mathbb{T}^{2}$ na classe de isotopia da identidade. Sejam $f: \mathbb{T}^{2} \rightarrow \mathbb{T}^{2}$ um homeomorfismo isotópico à identidade, onde $\mathbb{T}^{2}=\mathbb{R}^{2} / \mathbb{Z}^{2}, \tilde{f}: \mathbb{R}^{2} \rightarrow \mathbb{R}^{2}$ um levantamento de $f$ para o recobrimento universal de $\mathbb{T}^{2}$ e $\pi: \mathbb{R}^{2} \rightarrow \mathbb{T}^{2}$ a aplicação de recobrimento. Se $x \in \mathbb{T}^{2}$, denotamos por $\tilde{x}$ um levantamento de $x$ para $\mathbb{R}^{2}$, isto é, $\tilde{x} \in \pi^{-1}(x)$.

Definição 2.4 ([MZ89]). Chamamos o conjunto

$$
\rho(\tilde{f})=\left\{v \in \mathbb{R}^{2} \mid \exists \tilde{x}_{i} \in \mathbb{R}^{2}, \exists n_{i} \rightarrow \infty, \text { tais que } \frac{\tilde{f}^{n_{i}}\left(\tilde{x}_{i}\right)-\tilde{x}_{i}}{n_{i}} \rightarrow v\right\}
$$


de conjunto de rotação de $\tilde{f}$.

Em outras palavras, $\rho(\tilde{f})$ é o conjunto dos pontos de acumulação das sequências do tipo

$$
\left(\frac{\tilde{f}^{n_{i}}\left(\tilde{x}_{i}\right)-\tilde{x}_{i}}{n_{i}}\right)_{i \in \mathbb{N}}
$$

$\operatorname{com} \tilde{x}_{i} \in \mathbb{R}^{2}$ e $n_{i} \rightarrow \infty$.

Tal conjunto também é conhecido como conjunto de rotação de Misiurewicz-Ziemian (veja [MZ89]). Existem outras definições para conjuntos de rotação, contudo a definição aqui apresentada tem se mostrado a mais útil no estudo da dinâmica de homeomorfismos do toro.

Vale notar que se $\tilde{f}^{\prime}$ é outro levantamento de $f$, então $\tilde{f}^{\prime}=\tilde{f}+p$, para algum $p \in \mathbb{Z}^{2}$, e portanto $\rho\left(\tilde{f}^{\prime}\right)=\rho(\tilde{f})+p$. Portanto podemos falar simplesmente no conjunto de rotação de $f$. Note ainda que $\rho\left(\tilde{f}^{n}\right)=n \rho(\tilde{f})$, portanto se $\rho(\tilde{f})=\{(p / q, r / q)\}$, então $\rho\left(\tilde{f}^{q}-(p, r)\right)=\{0\}$.

À primeira vista a definição pode parecer desnecessariamente complicada se comparada ao caso do círculo, portanto vamos analisar de onde surge tal formulação. Considere um levantamento $\tilde{f}$ de $f$ e a função contínua $\phi: \mathbb{T}^{2} \rightarrow \mathbb{R}^{2}$ dada por $\phi(x)=\tilde{f}(\tilde{x})-\tilde{x}$, onde $\tilde{x} \in \pi^{-1}(x)$. Note que a função $\phi$ está bem definida (isto é, não depende da escolha do levantamento $\tilde{x}$ de $x$ ), pois, se tomarmos outro levantamento $\tilde{x}^{\prime} \in \pi^{-1}(x)$, teremos $\tilde{x}^{\prime}=\tilde{x}+k$, para algum $k \in \mathbb{Z}^{2}$, portanto

$$
\tilde{f}\left(\tilde{x}^{\prime}\right)-\tilde{x}^{\prime}=\tilde{f}(\tilde{x}+k)-(\tilde{x}+k)=\tilde{f}(\tilde{x})+k-\tilde{x}-k=\tilde{f}(\tilde{x})-\tilde{x} .
$$

Temos que

$$
\frac{1}{n} \sum_{k=0}^{n-1} \phi\left(f^{k}(x)\right)=\frac{1}{n} \sum_{k=0}^{n-1}\left(\tilde{f}^{k+1}(\tilde{x})-\tilde{f}^{k}(\tilde{x})\right)=\frac{\tilde{f}^{n}(\tilde{x})-\tilde{x}}{n} .
$$

Pelo Teorema Ergódico de Birkhoff (veja [Wal00]), se $\mu$ é uma medida de probabilidade em $\mathbb{T}^{2}$ invariante por $f$ e ergódica, então

$$
\lim _{n \rightarrow \infty} \frac{1}{n} \sum_{k=0}^{n-1} \phi\left(f^{k}(x)\right)=\int_{\mathbb{T}^{2}} \phi \mathrm{d} \mu \quad \text { para } \mu \text {-q.t.p. } x \in \mathbb{T}^{2},
$$

portanto

$$
\lim _{n \rightarrow \infty} \frac{\tilde{f}^{n}(\tilde{x})-\tilde{x}}{n}=\int_{\mathbb{T}^{2}} \phi \mathrm{d} \mu,
$$

sendo tal limite válido para $\mu$-q.t.p. $x \in \mathbb{T}^{2}$ e todo $\tilde{x} \in \pi^{-1}(x)$. É possível mostrar que

$$
\rho(\tilde{f})=\left\{\int_{\mathbb{T}^{2}} \phi \mathrm{d} \mu \mid \mu \text { é medida invariante por } f\right\}
$$

(veja [MZ89]). A ideia por trás é que o conjunto de rotação não mede apenas a velocidade de órbitas, isto é, ele não é a união das velocidades de todas as órbitas, mas também como $f$ faz todos os pontos rodarem.

Definição 2.5. Dizemos que $x \in \mathbb{T}^{2}$ tem vetor de rotação $v$ se

$$
\rho(\tilde{f}, x)=\lim _{n \rightarrow \infty} \frac{\tilde{f}^{n}(\tilde{x})-\tilde{x}}{n}=v,
$$

$\operatorname{com} \tilde{x} \in \pi^{-1}(x)$.

Note que, ao contrário do que ocorre no caso de $\mathbb{S}^{1}$, o limite da definição anterior pode não exisitir, e existem exemplos de tal comportamento. Note ainda que o limite, quando existe, não depende do levantamento de $x$ escolhido.

Temos duas perguntas naturais que surgem ao definirmos o conjunto de rotação: 
(1) Quais conjuntos podem ser realizados como conjunto de rotação para algum homeomorfismo?

(2) Dado que uma aplicação tem um determinado conjunto de rotação, o que se pode dizer sobre as propriedades dinâmicas de tal aplicação?

Vamos começar pela primeira questão. Um resultado inicial importante estabelece algumas restrições sobre quais conjuntos do plano podem ser conjuntos de rotação:

Teorema 2.6 ([MZ89]). O conjunto $\rho(\tilde{f})$ é compacto e convexo.

Evidentemente, temos que $\operatorname{int}(\rho(\tilde{f}))$ pode ser vazio ou não. Os dois principais exemplos de conjuntos de rotação com interior não vazio podem ser encontrados em [Kwa92], onde é provado que todo polígono convexo com vértices de coordenadas racionais é realizado como conjunto de rotação, e [Kwa95], onde um exemplo similar é construído, mas com infinitos vértices. Outro caso de interesse seria um conjunto de interior não vazio que não seja um polígono, como uma bola, por exemplo. Uma resposta, seja positiva ou negativa, ainda não foi encontrada. Para outros resultados mais recentes veja também [BdCTH16].

Em [FM90] foi conjecturado que se $\rho(\tilde{f})$ tem interior vazio então o conjunto só pode ser de uma das seguintes formas:

(i) um único ponto;

(ii) um segmento que contenha dois pontos racionais;

(iii) um segmento de inclinação irracional com um ponto racional extremal.

Note que é simples construir exemplos para os dois primeiros casos. Um exemplo para o terceiro caso pode ser encontrado em [Han89]. Outra forma de enunciar tal conjectura é, se $\rho(\tilde{f})$ tem interior vazio, então $\rho(\tilde{f})$ não pode ser das seguintes formas:

(i’) um segmento com inclinação racional e nenhum ponto racional;

(ii’) um segmento com inclinação irracional e nenhum ponto racional;

(iii') um segmento de inclinação irracional com um ponto racional no interior relativo do segmento.

Em [FM90] a conjectura foi provada para o caso em que $f$ é tempo 1 de um fluxo. No caso geral foi provado por Artur Avila que o caso (ii') é falso. Já o caso (iii') é verdadeiro, como visto em [CT18a]. O caso (i') permanece sem solução até o presente momento, contudo avanços têm sido feitos recentemente, veja [Koc16], [KPS16] e [PS18].

Vamos agora abordar a segunda questão: quais propriedades dinâmicas de uma aplicação o conjunto de rotação nos oferece? Temos os seguintes resultados iniciais sobre realização de vetores de rotação:

Teorema 2.7 ([LM91]). Se $v \in \operatorname{int}(\rho(\tilde{f}))$ então existe $x \in \mathbb{T}^{2}$ tal que $v=\rho(\tilde{f}, x)$.

Teorema 2.8 ([MZ91]). Se $v \in \operatorname{int}(\rho(\tilde{f}))$ então

(a) existe $X \subset \mathbb{T}^{2}$ um conjunto não vazio, fechado e $f$-invariante tal que $\rho(\tilde{f}, x)=\{v\}$, para todo $x \in X$;

(b) existe uma medida de probabilidade invariante por $f$ e ergódica tal que $\int_{\mathbb{T}^{2}} \phi \mathrm{d} \mu=v$, onde $\phi(x)=\tilde{f}(\tilde{x})-\tilde{x}$.

Temos, pela igualdade em (2.1), que o resultado de [LM91] também é válido se $v$ é um ponto extremal do conjunto de rotação, pois os pontos extremais do conjunto de rotação são realizados por medidas ergódicas (veja também [Fra88]). Em particular, em [MZ89] é provado também que pontos extremais com coordenadas racionais são realizados por pontos periódicos. Tal resultado nos leva ao seguinte questionamento: quando a existência de um vetor de rotação com coordenadas racionais implica a existência de um ponto periódico? Não existe um resultado geral sobre o assunto, mas temos alguns resultados que tratam de casos específicos. 
Teorema 2.9 ([Fra89]). Se $v \in \operatorname{int}(\rho(\tilde{f})) \cap \mathbb{Q}^{2}$, então existe $x \in \mathbb{T}^{2}$ periódico tal que $\rho(\tilde{f}, x)=v$.

Outro resultado importante, relacionado ao problema do presente trabalho, é o seguinte:

Teorema 2.10 ([JZ98]). Se $\rho(\tilde{f})$ é um segmento de reta com inclinação irracional e $v \in \rho(\tilde{f}) \cap \mathbb{Q}^{2}$, então existe $x \in \mathbb{T}^{2}$ ponto periódico tal que $\rho(\tilde{f}, x)=v$.

Note que é possível obter o resultado do teorema anterior da seguinte forma alternativa, usando trabalhos recentes: como discutido anteriormente, temos que se o conjunto de rotação é um segmento de inclinação irracional, em [CT18a] foi provado que se existe $v \in \rho(\tilde{f}) \cap \mathbb{Q}^{2}$, então $v$ é um ponto extremal. Assim, aplicando um resultado de [Fra88] obtemos um ponto periódico com vetor de rotação $v$.

Existe um resultado geral para conjuntos de rotação que são intervalos, contudo tal teorema carrega uma hipótese extra:

Teorema 2.11 ([Fra95]). Seja $f: \mathbb{T}^{2} \rightarrow \mathbb{T}^{2}$ um homeomorfismo homotópico à identidade que preserva área e $\tilde{f}: \mathbb{R}^{2} \rightarrow \mathbb{R}^{2}$ um levantamento. Se $\rho(\tilde{f})$ é um intervalo e $v \in \rho(\tilde{f}) \cap \mathbb{Q}^{2}$, então existe $x \in \mathbb{T}^{2}$ periódico tal que $v=\rho(\tilde{f}, x)$

Outra informação relevante que o conjunto de rotação fornece é sobre a entropia de $f$.

Teorema 2.12 ([LM91]). Se int $(\rho(\tilde{f})) \neq \emptyset$, então f tem entropia topológica positiva.

\subsection{Difusão Sublinear}

Vamos agora apresentar o conceito central tratado no teorema principal deste trabalho. Primeiramente, notemos que o conjunto de rotação mede a velocidade linear das órbitas. Mais precisamente, suponhamos que o ponto $x \in \mathbb{T}^{2}$, tenha vetor de rotação $v$, isto é, $\lim _{n \rightarrow \infty} \frac{\tilde{f}^{n}(\tilde{x})-\tilde{x}}{n}=v$. Por definição, temos que dado um $\varepsilon>0$, existe $n_{0} \in \mathbb{N}$ tal que

$$
\left|\frac{\tilde{f}^{n}(\tilde{x})-\tilde{x}}{n}-v\right|<\varepsilon, \quad \forall n \geq n_{0}
$$

e multiplicando a desigualdade por $n$ obtemos

$$
\left|\tilde{f}^{n}(\tilde{x})-\tilde{x}-n v\right|<n \varepsilon, \quad \forall n \geq n_{0} .
$$

Portanto, notando o termo $n \varepsilon$ na desigualdade, vemos como o conjunto de rotação mede a velocidade média linear.

Uma pergunta natural seria: existe algum tipo de velocidade menor que linear que o conjunto de rotação não é capaz de detectar? Além disso, se um ponto tem vetor de rotação $v$, o que podemos dizer das velocidades em outras direções, como $v^{\perp}$, por exemplo? As respostas dependem de como é o conjunto $\rho(\tilde{f})$.

Antes de passarmos aos casos para homeomorfismos do toro, vamos analisar qual a situação para homeomorfismos do círculo. Se $h: \mathbb{S}^{1} \rightarrow \mathbb{S}^{1}$ é um homeomorfismo do círculo que preserva orientação, sabemos que o número de rotação está bem definido e não depende da escolha do ponto onde é calculado. Além disso, é possível provar que

$$
\left|\tilde{h}^{n}(\tilde{z})-\tilde{z}-n \rho(\tilde{h})\right| \leq 1, \quad \forall n \in \mathbb{N}, \forall \tilde{z} \in \mathbb{R}^{2} .
$$

Portanto, não há difusão sublinear neste caso, ou seja, a distância entre $\tilde{h}^{n}(\tilde{z})-\tilde{z}$ e $n \rho(\tilde{h})$ fica uniformemente limitada. Intuitivamente, isso quer dizer que o conjunto de rotação captura de forma satisfatória as principais informações sobre a dinâmica de $h$.

No caso do toro, comecemos definindo o conceito de desvio limitado. 
Definição 2.13. Sejam $x \in \mathbb{T}^{2}$ e $v \in \mathbb{R}^{2}, v \neq 0$. Diremos que a órbita de $x$ tem desvio limitado na direção de $\vec{v}$ (ou não possui difusão sublinear na direção de $\vec{v}$ ) se existe $L>0$ tal que

$$
\left|\left\langle\tilde{f}^{n}(\tilde{x})-\tilde{x}, v\right\rangle\right|<L, \quad \forall n \in \mathbb{Z} .
$$

Diremos ainda que $f$ possui desvios uniformemente limitados se

$$
d\left(\tilde{f}^{n}(\tilde{x})-\tilde{x}, n \rho(\tilde{f})\right), \quad \forall \tilde{x} \in \mathbb{R}^{2}, \forall n \in \mathbb{Z}
$$

Vejamos agora alguns resultados sobre difusão sublinear e propriedades similares, dependendo do tipo de conjunto de rotação.

\subsubsection{Caso I: Apenas um ponto}

Vejamos primeiramente o caso mais simples, onde o conjunto de rotação é constituído de apenas um ponto.

Definição 2.14. Se $\rho(\tilde{f})=\{v\}$, diremos que $f$ é uma pseudo-rotação.

Se $f$ é uma pseudo-rotação, o caso mais simples possível seria $\rho(\tilde{f})=\{0\}$, que é conhecido como um homeomorfismo irrotacional. Esse é um caso onde é possível construir um exemplo que apresenta difusão sublinear. Antes de apresentarmos o enunciado, vejamos uma definição necessária para tal.

Definição 2.15. Seja $X \subset \mathbb{R}^{2}$. Diremos que $X$ se acumula numa direção $v \in \mathbb{S}^{1}$ no infinito se existe uma sequência $\left(x_{n}\right)_{n \in \mathbb{N}}$ em $X$ tal que

$$
\lim _{n \rightarrow \infty}\left\|x_{n}\right\|=\infty \quad \text { e } \quad \lim _{n \rightarrow \infty} \frac{x_{n}-x_{0}}{\left\|x_{n}-x_{0}\right\|}=v .
$$

A fronteira de $X$ no infinito é definida como o conjunto $\partial_{\infty} X \subset \mathbb{S}^{1}$ de todas as direções $v$ tais que $X$ se acumula em $v$ no infinito.

O enunciado preciso é o seguinte:

Teorema 2.16 ([KT14a]). Existe um difeomorfismo homotópico à identidade $f: \mathbb{T}^{2} \rightarrow \mathbb{T}^{2}$, de classe $\mathcal{C}^{\infty}$ e que preserva área, com um levantamento $\tilde{f}: \mathbb{R}^{2} \rightarrow \mathbb{R}^{2}$ tal que:

(i) $\rho(\tilde{f})=\{0\}$;

(ii) $f$ é metricamente isomorfa à um shift de Bernoulli com respeito à medida de Lebesgue (em particular, $f$ é ergódica);

(iii) Para Lebesgue quase todo ponto $\tilde{x} \in \mathbb{R}^{2}$, tanto a órbita passada quanto a órbita futura de $\tilde{x}$ se acumulam em toda direção no infinito, isto é,

$$
\partial_{\infty}\left\{\tilde{f}^{n}(\tilde{x}) \mid n \in \mathbb{N}\right\}=\mathbb{S}^{1}=\partial_{\infty}\left\{\tilde{f}^{-n}(\tilde{x}) \mid n \in \mathbb{N}\right\}
$$

Além disso, tanto a órbita passada quanto a órbita futura de $\tilde{x}$ visitam todo domínio fundamental $[0,1]^{2}+v$, com $v \in \mathbb{Z}^{2}$.

Note que $\tilde{f}$ como no teorema acima possui desvio ilimitado em todas as direções.

Existem resultados na direação contrária, que dão condições para a limitação das órbitas no caso em que $\rho(\tilde{f})=\{0\}$. Um primeiro resultado parcial pode ser encontrado em [KT14b], e um resultado com hipóteses mais abrangentes é o seguinte:

Teorema 2.17 ([CT18a]). Seja $f: \mathbb{T}^{2} \rightarrow \mathbb{T}^{2}$ um homeomorfismo homotópico à identidade tal que $f$ preserva uma medida $\mu$ de suporte total e tal que existe $\tilde{f}: \mathbb{R}^{2} \rightarrow \mathbb{R}^{2}$ um levantamento de $f$ 
de forma que $\int_{\mathbb{T}^{2}}(\tilde{f}(\tilde{x})-\tilde{x}) \mathrm{d} \mu=0$. Se todos os pontos periódicos de $f$ forem contráteis e se o conjunto dos pontos fixos de $f$ estiver contido em um disco topológico, então existe $K>0$ tal que $\left\|\tilde{f}^{n}(\tilde{x})-\tilde{x}\right\| \leq K$, para todo $n \geq 1$ e todo $\tilde{x} \in \mathbb{R}^{2}$.

Uma pergunta relacionada ao teorema anterior que permenece em aberto é se tal resultado é válido se a hipótese de preservação de uma medida de suporte total for retirada.

No teorema anterior, um ponto periódico em $\mathbb{T}^{2}$ é dito contrátil se seu caminho da isotopia for homotopicamente trivial em $\mathbb{T}^{2}$ (para a definição de caminho da isotopia, veja o Capítulo 3) ou, equivalentemente, se o vetor de rotação de tal ponto for nulo.

Até aqui, vimos resultados tratando do caso do conjunto de rotação ser apenas o ponto 0 (note que se $\rho(\tilde{f})=\{(p / q, r / q)\}$, então $\left.\rho\left(\tilde{f}^{q}-(p, r)\right)=\{0\}\right)$. Vejamos agora um resultado de classificação de pseudo-rotações com movimento médio limitado, que inclui o caso onde o vetor de rotação não é racional:

Teorema 2.18 ([Jäg09b]). Se f é uma pseudo-rotação conservativa (isto é, existe uma medida de probabilidade de suporte total invariante por $f$ ) com vetor de rotação $\rho \in \mathbb{R}^{2}$ e tal que existe $c>0$ de forma que $\left\|\tilde{f}^{n}(\tilde{x})-\tilde{x}-n \rho\right\| \leq c$, para todo $n \in \mathbb{Z}$ e todo $\tilde{x} \in \mathbb{R}^{2}$, então vale:

(i) $\rho$ é totalmente irracional se e somente se $f$ é semiconjugada à rotação por $\rho$;

(ii) $\rho$ não é totalmente irracional e nem racional se e somente se f possui um circlóide periódico

(iii) $\rho$ é racional se e somente se f possui um ponto periódico.

Veja também [Jäg09a] para outros resultados sobre pseudo-rotações.

\subsubsection{Caso II: Interior não vazio}

Suponhamos agora que o interior do conjunto $\rho(\tilde{f})$ tenha interior não vazio. Temos aqui uma resposta completa ao problema da difusão sublinear:

Teorema 2.19 ([CT18a]). Se $\operatorname{int}(\rho(\tilde{f})) \neq \emptyset$, existe $L \geq 0$ tal que

$$
d\left(\tilde{f}^{n}(\tilde{x})-\tilde{x}, n \rho(\tilde{f})\right) \leq L, \quad \forall \tilde{x} \in \mathbb{R}^{2}, \forall n \geq 1 .
$$

Ressaltamos que o resultado acima foi obtido anteriormente no caso de $\rho(\tilde{f})$ ser um polígono não degenerado de vértices racionais, veja [Dáv16], e no caso de $f$ ser um difeomorfismo $\mathcal{C}^{1+\varepsilon}$, veja [AZ15].

\subsubsection{Caso III: Segmento de reta}

Vejamos agora qual tipo de resultado já foi obtido quando o conjunto de rotação é um segmento de reta. Um primeiro teorema é o seguinte:

Teorema 2.20 ([GKT14]). Seja $f: \mathbb{T}^{2} \rightarrow \mathbb{T}^{2}$ é um homeomorfismo homotópico à identidade que preserva uma medida de probabilidade boreleana de suporte total. Se $\tilde{f}: \mathbb{R}^{2} \rightarrow \mathbb{R}^{2}$ é um levantamento de $f$ tal que $\rho(\tilde{f})=\{0\} \times[a, b]$, com $a<b$, então existe $M>0$ tal que $\left\langle\tilde{f}^{n}(\tilde{x})-\tilde{x},(1,0)\right\rangle \leq M$, para todo $\tilde{x} \in \mathbb{R}^{2}$ e todo $n \in \mathbb{Z}$. Além disso, existe um anel essencial invariante (que é necessariamente vertical).

Note que o teorema anterior vale para qualquer segmento com dois pontos de coordenadas racionais: de fato, se $A \in S L(2, \mathbb{Z})$, temos que $\rho\left(A \tilde{f} A^{-1}\right)=A \rho(\tilde{f})$, portanto se $\rho(\tilde{f})$ é um segmento contendo dois pontos de $\mathbb{Q}^{2}$, podemos encontrar uma matriz $A \in S L(2, \mathbb{Z})$ de forma que $A \rho(\tilde{f})$ seja um segmento vertical, como no teorema anterior.

Outro resultado mais geral desse caso é o próximo teorema. 
Teorema 2.21 ([Dáv16]). Se $\rho(\tilde{f})$ é um segmento de reta contendo dois pontos racionais, então existem $k \in \mathbb{N}, F: \mathbb{R}^{2} \rightarrow \mathbb{R}^{2}$ um levantamento de $f^{k}$ e $M>0$ tais que

$$
\left|\left\langle F^{n}(\tilde{x})-\tilde{x}, v^{\perp}\right\rangle\right| \leq M, \quad \forall \tilde{x} \in \mathbb{R}^{2}, \forall n \in \mathbb{Z},
$$

sendo que $v$ é um vetor tal que $\rho(\tilde{f})=\{t v+A \mid t \in[0,1]\}$, para algum $A \in \mathbb{R}^{2}$.

Portanto, vemos que nesse caso não existe difusão sublinear na direção ortogonal à direção dada pelo conjunto de rotação. Intuitivamente, podemos dizer que o conjunto de rotação apresenta uma boa representação da dinâmica de $f$.

Existem outros trabalhos que analisam o caso em que o conjunto de rotação é um segmento de reta, sob diversos aspectos. Em [KR17], os autores demonstram uma condição necessária e suficiente, sob determinadas hipóteses, para um homeomorfismo do toro não apresentar difusão sublinear.

Um resultado interessante apresentado em [Koc16] é o seguinte: se $f$ for um homeomorfismo mininal cujo conjunto de rotação não é apenas um ponto, então tal homeomorfismo apresenta desvio limitado na direção perpendicular ao conjunto de rotação.

Outro resultado interessante pode ser encontrado em [JT17], onde os autores encontram condições necessárias e suficientes para um homeomorfismo do toro, sob determinadas hipóteses, ser semiconjugado a uma rotação irracional no círculo, e tais condições envolvem a inexistência de difusão sublinear em determinadas direções.

Outro caso possível para o conjunto de rotação é ser um segmento de reta com inclinação irracional e um ponto extremal de coordenadas racionais, que será o caso tratado neste trabalho. Note que tal caso era o único que restava sem solução, entre os conjuntos de rotação que sabidamente são realizados por algum homeomorfismo do toro. O resultado obtido é o seguinte:

Teorema A. Suponhamos que $\rho(\tilde{f})=\left\{t \rho_{0} \mid 0 \leq t \leq 1\right\}$, onde $\tan \left(\rho_{0}\right) \notin \mathbb{Q}$. Então existe $M>0$ tal que

$$
\left|\left\langle\tilde{f}^{n}(\tilde{x})-\tilde{x}, \rho_{0}^{\perp}\right\rangle\right|<M,
$$

para todo $\tilde{x} \in \mathbb{R}^{2}$ e $n \in \mathbb{Z}$.

\subsubsection{Dehn twists}

Um caso importante que não será tratado neste trabalho é o caso em que $f: \mathbb{T}^{2} \rightarrow \mathbb{T}^{2}$ é um homeomorfismo homotópico à um Dehn twist (um Dehn twist é uma aplicação do tipo $(x, y) \mapsto$ $(x+k y \bmod 1, y \bmod 1)$ para algum $\left.k \in \mathbb{Z}_{*}\right)$. É possível definir um conjunto de rotaçâa vertical nesse caso, para um levantamento $\hat{f}$ de $f$ para o cilindro $\mathbb{S}^{1} \times \mathbb{R}$, denotado por $\rho_{V}(\hat{f})$. Existem resultados similares aos listados aqui, mas no contexto de Dehn twists, veja por exemplo [AZTG12].

\subsection{Dinâmica Essencial}

Vejamos agora uma ferramenta que nos auxiliará no demonstração do resultado principal deste trabalho. As referências da teoria a seguir são [KT14c] e [KT18], contudo aqui iremos apresentar apenas uma pequena parte necessária para o desenvolvimento do nosso trabalho, sendo os principais resultados a Proposição 2.24 e o Lema 2.25.

Comecemos com algumas definições:

Definição 2.22. Diremos que um conjunto aberto $U \subset \mathbb{T}^{2}$ é inessencial se todo caminho fechado contido em $U$ é homotopicamente trivial em $\mathbb{T}^{2}$, caso contrário, $U$ será dito essencial. Diremos que um conjuto qualquer $E \subset \mathbb{T}^{2}$ é inessencial se possuir uma vizinhança inessencial. Além disso, diremos que $E$ é totalmente essencial se $\mathbb{T}^{2} \backslash E$ é inessencial.

Definição 2.23. Seja $x \in \mathbb{T}^{2}$ e $f: \mathbb{T}^{2} \rightarrow \mathbb{T}^{2}$ um homeomorfismo homotópico a identidade. Diremos que $x$ é um ponto inessencial de $f$ se $\cup_{k \in \mathbb{Z}} f^{k}(U)$ é inessencial para alguma vizinhança $U$ de $x$. Se $x$ não for inessencial, diremos que $x$ é um ponto essencial de $f$, ou seja, $\cup_{k \in \mathbb{Z}} f^{k}(U)$ é essencial para 
toda vizinhança $U$ de $x$. Diremos ainda que $x$ é um ponto totalmente essencial de $f$ se $\cup_{k \in \mathbb{Z}} f^{k}(U)$ é totalmente essencial para toda vizinhança $U$ de $x$.

Na seguinte proposição (veja [GKT15]) a norma $\|x\|_{\infty}$ é definada por $\|x\|_{\infty}=\max \left\{\left|(x)_{1}\right|,\left|(x)_{2}\right|\right\}$, onde $(x)_{1}$ e $(x)_{2}$ denotam a primeira e segunda coordenadas, respectivamente, de um ponto $x \in \mathbb{R}^{2}$.

Proposição 2.24 ([GKT15]). Seja $O \subset \mathbb{R}^{2}$ um aberto conexo tal que $\bigcup_{n \in \mathbb{Z}} f^{n}(\pi(O))$ é totalmente essencial e $\overline{\pi(O)}$ é inessencial. Então existem $M \in \mathbb{N}$ e $K \subset \mathbb{R}^{2}$ compacto tais que $[0,1]^{2}$ está contido numa componente conexa limitada de $\mathbb{R}^{2} \backslash K e$

$$
K \subset \bigcup_{|i| \leq M,\|v\|_{\infty} \leq M}\left(\tilde{f}^{i}(O)+v\right) .
$$

Demonstração. Como $\cup_{n \in \mathbb{Z}} f^{n}(\pi(O))$ é totalmente essencial, sua pré-imagem por $\pi$, que denotaremos por $\tilde{U}$, é um conjunto conexo de $\mathbb{R}^{2}$ invariante por translações de $\mathbb{Z}^{2}$. Assim, dado $\tilde{y} \in \tilde{U}$, existem dois $\operatorname{arcos} \tilde{\alpha}, \tilde{\beta}:[0,1] \rightarrow \tilde{U}$, tais que $\tilde{\alpha}$ conecta $\tilde{y}$ a $\tilde{y}+(1,0)$ e $\tilde{\beta}$ conecta $\tilde{y}$ a $\tilde{y}+(0,1)$. Sejam

$$
\tilde{\Gamma}_{\tilde{\alpha}}=\bigcup_{i=-\infty}^{+\infty}([\tilde{\alpha}]+(i, 0)), \quad \tilde{\Gamma}_{\tilde{\beta}}=\bigcup_{i=-\infty}^{+\infty}([\tilde{\beta}]+(0, i)),
$$

onde $[\tilde{\alpha}]=\tilde{\alpha}([0,1])$ e $[\tilde{\beta}]=\tilde{\beta}([0,1])$, e note que, como $\tilde{U}$ é invariante por translações de $\mathbb{Z}^{2}$, $\tilde{\Gamma}_{\tilde{\alpha}}, \tilde{\Gamma}_{\tilde{\beta}} \subset \tilde{U}$, bem como todos os translados inteiros dos dois conjuntos também estão contidos em $\tilde{U}$.

Fixemos um inteiro $R>\max \{\|x\| \mid x \in[\tilde{\alpha}] \cup[\tilde{\beta}]\}$. Então, como $\max \left\{(x)_{2} \mid x \in \tilde{\Gamma}_{\tilde{\alpha}}\right\}=$ $\max \left\{(x)_{2} \mid x \in[\tilde{\alpha}]\right\}<R$ e $\min \left\{(x)_{2} \mid x \in \tilde{\Gamma}_{\tilde{\alpha}}\right\}>-R$, temos que $\mathbb{R}^{2} \backslash \tilde{\Gamma}_{\tilde{\alpha}}$ possui ao menos duas componentes conexas, uma contendo o semi-plano $\left\{x \mid(x)_{2} \geq R\right\}$ e outra contendo o semiplano $\left\{x \mid(x)_{2} \leq-R\right\}$. Analogamente, $\mathbb{R}^{2} \backslash \tilde{\Gamma}_{\tilde{\beta}}$ possui ao menos duas componentes conexas, uma contendo $\left\{x \mid(x)_{1} \geq R\right\}$ e outra contendo $\left\{x \mid(x)_{1} \leq-R\right\}$.

Agora seja

$$
F=\left(\tilde{\Gamma}_{\tilde{\alpha}}-(0, R)\right) \cup\left(\tilde{\Gamma}_{\tilde{\alpha}}+(0, R+1)\right) \cup\left(\tilde{\Gamma}_{\tilde{\beta}}-(R, 0)\right) \cup\left(\tilde{\Gamma}_{\tilde{\beta}}+(R+1,0)\right),
$$

e note que $F \subset \tilde{U}$ e $\mathbb{R}^{2} \backslash F$ possui uma componente conexa $W$ que contém [0,1] $]^{2}$ e está contida em $[-2 R, 2 R+1] \times[-2 R, 2 R+1]$.

Seja agora $K=\partial W$. Assim, $K$ é um subconjunto compacto de $\tilde{U}$. Como $\tilde{U}=\cup_{v \in \mathbb{Z}^{2}} \cup_{i \in \mathbb{Z}}$ $\left(\tilde{f}^{i}(O)+v\right)$ é uma cobertura por abertos de $K$, escolhendo uma subcobertura finita podemos concluir a existência de $M$.

Lema 2.25. Sejam $f: \mathbb{T}^{2} \rightarrow \mathbb{T}^{2}$ um homeomorfismo homotópico a identidade, $\tilde{f}: \mathbb{R}^{2} \rightarrow \mathbb{R}^{2}$ um levantamento e $z_{0} \in \mathbb{T}^{2}$ um ponto recorrente tal que $\rho\left(\tilde{f}, z_{0}\right)$ é totalmente irracional. Então $z_{0}$ é um ponto totalmente essencial de $f$, isto é, para todo $\varepsilon>0$, o conjunto $U_{\varepsilon}=\bigcup_{i=0}^{\infty} f^{i}\left(\pi\left(B\left(\varepsilon, \tilde{z}_{0}\right)\right)\right)$ é totalmente essencial.

Demonstração. Se $z_{0}$ for inessencial, temos que existe $\varepsilon>0$ tal que $U_{\varepsilon}=\bigcup_{i=0}^{\infty} f^{i}\left(\pi\left(B\left(\varepsilon, \tilde{z}_{0}\right)\right)\right)$ é inessencial. Veja que cada componente conexa de $U_{\varepsilon}$ está contida em um disco topológico. Como $U_{\varepsilon}$ é $f$-invariante, temos que $f$ permuta as componentes conexas de $U_{\varepsilon}$. Assim, como $z_{0}$ é recorrente, sendo $U_{\varepsilon}^{0}$ a componente conexa de $U_{\varepsilon}$ que contém $z_{0}$, temos que existe $N>0$ tal que $f^{N}\left(U_{\varepsilon}^{0}\right)=U_{\varepsilon}^{0}$, e podemos assumir que $N$ é o primeiro instante que a igualdade ocorre (isto é, $f^{i}\left(U_{\varepsilon}^{0}\right) \cap U_{\varepsilon}^{0}=\emptyset$, se $1 \leq i<N)$. Assim, temos que existe $w \in \mathbb{Z}^{2}$ tal que $\tilde{f}^{N}\left(\widetilde{U}_{\varepsilon}^{0}\right)=\widetilde{U}_{\varepsilon}^{0}+w$, onde $\widetilde{U}_{\varepsilon}^{0}$ é o levantamento de $U_{\varepsilon}^{0}$ que contém $\tilde{z}_{0}$. Como $z_{0}$ é recorrente, temos que existe subsequência $n_{k}$ tal que $f^{n_{k}}\left(z_{0}\right) \rightarrow z_{0}$. Assim, existe $k_{0}$ tal que se $k>k_{0}$ temos que $f^{n_{k}}\left(z_{0}\right) \in U_{\varepsilon}^{0}$. Mas como temos $f^{i}\left(U_{\varepsilon}^{0}\right) \cap U_{\varepsilon}^{0}=\emptyset$, se $1 \leq i<N$, temos que $n_{k}=p_{k} N$, para $k>k_{0}$. Assim, temos que $\tilde{f}^{n_{k}}\left(\tilde{z}_{0}\right)=\tilde{f}^{p_{k} N}\left(\tilde{z}_{0}\right) \in \tilde{f}^{p_{k} N}\left(\widetilde{U}_{\varepsilon}^{0}\right)=$ $\widetilde{U}_{\varepsilon}^{0}+p_{k} w$. Portanto, $\tilde{f}^{p_{k} N}\left(\tilde{z}_{0}\right)-p_{k} w \rightarrow \tilde{z}_{0}$, o que implica que $\rho\left(\tilde{f}, z_{0}\right)=w / N$, o que é um absurdo.

Suponhamos agora que $z_{0}$ seja essencial, mas não totalmente essencial, isto é, existe $\varepsilon>0$ tal que $U_{\varepsilon}=\bigcup_{i=0}^{\infty} f^{i}\left(\pi\left(B\left(\varepsilon, \tilde{z}_{0}\right)\right)\right)$ é essencial, mas não totalmente essencial. Assim, existe uma curva 
fechada homotopicamente não trivial em $\mathbb{T}^{2}$, isto é, $\alpha: \mathbb{R} \rightarrow \mathbb{T}^{2}$ tal que $\alpha(t)=\alpha(t+1)$, para todo $t \in \mathbb{R},[\alpha] \subset U_{\varepsilon}$, e $\tilde{\alpha}: \mathbb{R} \rightarrow \mathbb{R}^{2}$ um levantamento de $\alpha$ tal que $\tilde{\alpha}(t+1)=\tilde{\alpha}(t)+W$, para algum $W \in \mathbb{Z}_{*}^{2}$ e todo $t \in \mathbb{R}$. Seja $U_{\varepsilon}^{0}$ a componente conexa de de $U_{\varepsilon}$ que contém $[\alpha]$. Como $z_{0}$ não é totalmente essencial, temos que $\mathbb{T}^{2} \backslash U_{\varepsilon}^{0}$ também é um conjunto essencial. Como $z_{0}$ é recorrente, temos que existe $N \in \mathbb{N}$ tal que $f^{N}\left(U_{\varepsilon}^{0}\right)=U_{\varepsilon}^{0}$, portanto existem $\hat{f}: \mathbb{R}^{2} \rightarrow \mathbb{R}^{2}$ levantamento de $f$ e $\widehat{U}_{\varepsilon}^{0}$ levantamento de $U_{\varepsilon}^{0}$ tais que $\hat{f}^{N}\left(\widehat{U}_{\varepsilon}^{0}\right)=\widehat{U}_{\varepsilon}^{0}$, e temos também que $\hat{f}^{N}\left(\mathbb{R}^{2} \backslash \widehat{U}_{\varepsilon}^{0}\right)=\mathbb{R}^{2} \backslash \widehat{U}_{\varepsilon}^{0}$. Como $U_{\varepsilon}^{0}$ é essencial mas não totalmente essencial, temos que $\widehat{U}_{\varepsilon}^{0}$ é limitado na direção $W^{\perp}$, portanto $\rho(\hat{f}) \subset\{t W \mid t \in \mathbb{R}\}$, o que é um absurdo. 


\section{Capítulo 3}

\section{Folheação de Brouwer-Le Calvez e Forcing}

\subsection{Homeomorfismos de Brouwer}

Ao longo deste capítulo estudaremos homeomorfismos do plano sem pontos fixos e alguns resultados sobre tais aplicações que serão importantes para o prosseguimento do trabalho.

Definição 3.1. Um homeomorfismo $h: \mathbb{R}^{2} \rightarrow \mathbb{R}^{2}$ que preserva a orientação e que não possui pontos fixos $\left(h(x) \neq x, \forall x \in \mathbb{R}^{2}\right)$ será chamado de homeomorfismo de Brouwer.

Vejamos primeiramente uma definição que será usada ao longo do trabalho.

Definição 3.2. Uma linha de $\mathbb{R}^{2}$ é uma aplicação contínua $\phi: \mathbb{R} \rightarrow \mathbb{R}^{2}$ injetiva e própria (isto é, se $K \subset \mathbb{R}^{2}$ é compacto, então $\phi^{-1}(K)$ é compacto também).

Pelo Teorema de Schöenflies, temos que se $\phi: \mathbb{R} \rightarrow \mathbb{R}^{2}$ é uma linha, então a função $\phi$ pode ser estendida para um homeomorfismo $\phi^{*}: \mathbb{R}^{2} \rightarrow \mathbb{R}^{2}$ que preserva orientação, sendo que $\phi(t)=$ $\phi^{*}(t, 0)$. Assim, podemos definir a esquerda de $\phi$, denotada por $L(\phi)$, como $L(\phi)=\phi^{*}(\mathbb{R} \times(0,+\infty))$. Analogamente, definimos a direita de $\phi$, denotada por $R(\phi)$, como $R(\phi)=\phi^{*}(\mathbb{R} \times(-\infty, 0))$.

O principal resultado sobre homeomorfimos de Brouwer é o seguinte:

Teorema 3.3 ([Bro12]). Dados $h: \mathbb{R}^{2} \rightarrow \mathbb{R}^{2}$ um homeomorfismo de Brouwer e $x \in \mathbb{R}^{2}$, existe uma linha $\phi: \mathbb{R} \rightarrow \mathbb{R}^{2}$, com $\phi(0)=x$, tal que $h([\phi]) \subset L(\phi)$ e $h^{-1}([\phi]) \subset R(\phi)$, onde $[\phi]=\phi(\mathbb{R})$.

Uma linha como no teorema anterior será dita uma linha de Brouwer. Para uma demonstração mais recente veja também [Fra92]. Existem diversos resultados sobre homeomorfismos de Brouwer equivalentes ao Teorema 3.3. Vamos enunciar duas outras formas equivalentes abaixo.

Teorema 3.4 ([Bro12]). Dados $h: \mathbb{R}^{2} \rightarrow \mathbb{R}^{2}$ homeomorfismo de Brouwer e $x \in \mathbb{R}^{2}$, existe $U \subset \mathbb{R}^{2}$ aberto simplesmente conexo, com $x \in U$, tal que $h_{U}: U \rightarrow U$ é conjugada ao homeomorfismo do plano $g(x)=x+(1,0)$.

Um conjunto $U$ como no Teorema 3.4 será chamado um domínio de translação. Uma outra versão de tal resultado é a seguinte:

Teorema 3.5 ([Bro12]). Se $h: \mathbb{R}^{2} \rightarrow \mathbb{R}^{2}$ é um homeomorfismo de Brouwer, então todo ponto $x \in \mathbb{R}^{2}$ é errante. Em particular, $\Omega(h)=\emptyset$, onde $\Omega(h)$ é o conjunto dos pontos não-errantes de $h$.

Portanto um homeomorfismo de Brouwer não possui qualquer tipo de recorrência. 


\subsection{Folheação de Brouwer-Le Calvez}

Antes de apresentarmos a ferramenta principal utilizada na demonstração do Teorema A, que será introduzida na próxima seção, vejamos algumas definições e conceitos necessários para tal.

Definição 3.6. Seja $M$ uma superfície orientada. Uma folheação topológica orientada com singularidades em $M$ é uma folheação topológica orientada $\mathcal{F}$ definida em um subconjunto aberto de $M$. Tal conjunto será chamado domínio de $\mathcal{F}$ e será denotado por $\operatorname{dom}(\mathcal{F})$, o seu complemento será o conjunto das singularidade de $\mathcal{F}$, e será denotado por $\operatorname{sing}(\mathcal{F})$.

Como visto na seção anterior, se $h: \mathbb{R}^{2} \rightarrow \mathbb{R}^{2}$ é um homeomorfismo de Brouwer, por todo ponto do plano passa uma linha de Brouwer. Uma pergunta natural seria: qual tipo de relação podemos obter para linhas de Brouwer de pontos distintos? O próximo resultado mostra que é possível construir uma folheação topológica do plano onde cada folha é uma linha de Brouwer.

Teorema 3.7 ([Ca105]). Sejam $h: \mathbb{R}^{2} \rightarrow \mathbb{R}^{2}$ um homeomorfismo de Brouwer e $G$ um grupo discreto de homeomorfismos do plano que preservam orientação, que age livremente e propriamente em $\mathbb{R}^{2}$. Se $h$ comuta com os elementos de $G$, então existe $\mathcal{F}$ folheação $G$-invariante de $\mathbb{R}^{2}$ por linhas de Brouwer de $h$.

Chamaremos uma folheação $\mathcal{F}$ como no teorema acima de uma folheação de Brouwer-Le Calvez.

Observação 3.8. Evidentemente nosso interesse primário neste trabalho não serão homeomorfismos de Brouwer, contudo o Teorema 3.7 pode ser aplicado para homeomorfismos de superfície. Informalmente, se $f: M \rightarrow M$ é um homeomorfismo que preserva orientação (e $M$ uma superfície orientada), a ideia consiste em remover um subconjunto dos pontos fixos $f$ da superfície $M$, obtendo assim uma nova superfície, que denotaremos por $N$. Se $N$ for conexa, seu recobrimento universal será homeomorfo ao plano, e portanto podemos aplicar o Teorema 3.7 para o levantamento de $\left.f\right|_{N}$, e sendo $G$ o grupo de aplicações de recobrimento, obtemos uma folheação em $\mathbb{R}^{2}$, invariante por $G$, que se projeta em uma folheação em $N$, e portanto obteremos assim uma folheação com singularidades (os pontos fixos de $f$ que retiramos) em $M$. Ressaltamos que essa é apenas a ideia geral da construção, alguns cuidados precisam ser tomados na hora de determinar quais pontos fixos precisam ser retirados, mas a construção completa será apresentada com detalhes na próxima seção.

Iremos apresentar agora algumas definições e resultados sobre folheações que serão usadas no nosso trabalho. A referência para essa seção é [CT18a]. Denotaremos por $M$ uma superfície orientada e $\mathcal{F}$ uma folheação topológica orientada (possivelmente com singularidades) de $M$. Denotaremos também por $\phi_{z}$ a folha que passa pelo ponto $z$.

Definição 3.9. Um caminho em $M$ é uma aplicação $\gamma: I \rightarrow M$ contínua, onde $I \subset \mathbb{R}$ é um conjunto conexo contendo mais de dois pontos distintos. Denotaremos por $[\gamma]$ a imagem de $\gamma$, definida como $[\gamma]=\gamma(I)$.

Definição 3.10. Diremos que um caminho $\gamma: J \rightarrow \operatorname{dom}(\mathcal{F})$ é transverso a $\mathcal{F}$ se para todo $t \in J$ existe um homeomorfismo $c: W \rightarrow(0,1)^{2}$, onde $W$ é uma vizinhança de $\gamma(t)$, compatível com a orientação e tal que $c$ manda a restrição da folheação em $W$ na folheação vertical orientada para baixo em $(0,1)^{2}$ e $\pi_{1} \circ c \circ \gamma$ é estritamente crescente em uma vizinhança de $t$, onde $\pi_{1}$ é a projeção na primeira coordenada.

Intuitivamente, um caminho transverso atravessa as folhas sempre da direita para a esquerda (veja a Figura 3.1).

Se $M=\mathbb{R}^{2}$ e $\mathcal{F}$ não tem singularidades, diremos que duas curvas transversas são equivalentes se elas atravessam as mesmas folhas. No caso geral, temos o seguinte:

Definição 3.11. Dois caminhos transversos $\gamma: J \rightarrow \operatorname{dom}(\mathcal{F})$ e $\gamma^{\prime}: J^{\prime} \rightarrow \operatorname{dom}(\mathcal{F})$ são ditos equivalentes se existem $H: J \times[0,1] \rightarrow \operatorname{dom}(\mathcal{F})$ transformação contínua e $h: J \rightarrow J^{\prime}$ homeomorfismo crescente tais que: 


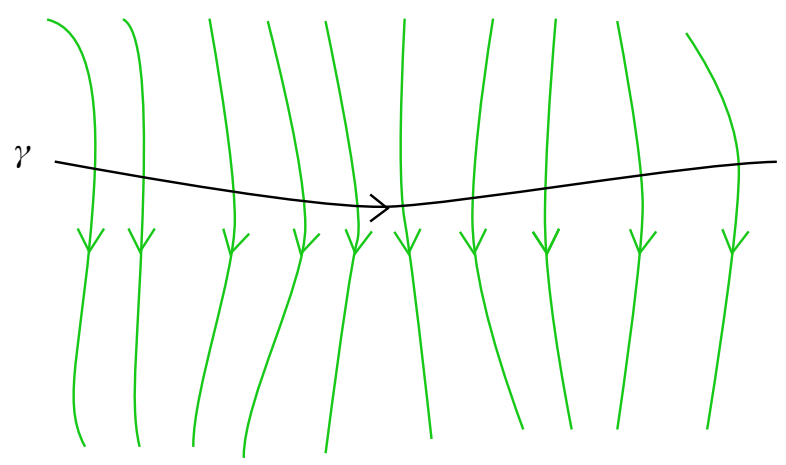

Figura 3.1: Um caminho transverso $\gamma$

(i) $H(t, 0)=\gamma(t), H(t, 1)=\gamma^{\prime}(h(t))$;

(ii) $\forall t \in J$ e $\forall s_{1}, s_{2} \in[0,1], \phi_{H\left(t, s_{1}\right)}=\phi_{H\left(t, s_{2}\right)}$.

Denotaremos $\gamma \sim_{\mathcal{F}} \gamma^{\prime}$.

A transformação $H$ é dita uma homotopia holonômica. Intuitivamente, uma homotopia holonômica deforma um caminho preservando cada ponto em sua folha original, isto é, ela move os pontos ao longo das folhas. É possível mostrar que a definição anterior é equivalente a existirem levantamentos de $\gamma$ e $\gamma^{\prime}$ no recobrimento universal de $\operatorname{dom}(\mathcal{F})$ que são equivalentes. Note ainda que duas curvas equivalentes atravessam as mesmas folhas, contudo tal condição não é suficiente para duas curvas serem equivalentes (veja o contraexemplo da Figura 3 de [CT18a]).

Observação 3.12. Note que se $\gamma: \mathbb{R} \rightarrow \mathbb{R}^{2}$ é uma linha transversa, então toda folha $\phi$ que intersecta $\gamma$ só pode intersectar a linha em um único ponto, e a folha $\phi$ cruza a linha $\gamma$ da esquerda para a direita, isto é, se $t^{\prime} \in \mathbb{R}$ é tal que $\phi\left(t^{\prime}\right) \in[\gamma]$, então $\phi(t) \in L(\gamma)$, se $t<t^{\prime}$, e $\phi(t) \in R(\gamma)$, se $t>t^{\prime}$.

Dadas três linhas $\gamma_{i}: \mathbb{R} \rightarrow \mathbb{R}^{2}, i \in\{0,1,2\}$, diremos que $\gamma_{0}$ separa $\gamma_{1}$ de $\gamma_{2}$ se $\gamma_{1}$ e $\gamma_{2}$ estiverem contidas em componentes conexas distintas de $\mathbb{R}^{2} \backslash\left[\gamma_{0}\right]$.

Definição 3.13. Seja $\gamma_{i}: \mathbb{R} \rightarrow \mathbb{R}^{2}$ uma linha, com $i \in\{0,1,2\}$. Diremos que $\gamma_{2}$ está acima de $\gamma_{1}$ com relação à $\gamma_{0}$ se forem satisfeitos:

(i) as três linhas são duas a duas disjuntas;

(ii) nenhuma das linhas separa as outras duas;

(iii) se $\lambda_{1}, \lambda_{2}$ são dois caminhos disjuntos ligando $z_{1}=\gamma_{0}\left(t_{1}\right), z_{2}=\gamma_{0}\left(t_{2}\right)$ a $z_{1}^{\prime} \in\left[\gamma_{1}\right], z_{2}^{\prime} \in\left[\gamma_{2}\right]$, respectivamente, e não intersectam as linhas, exceto pelos extremos, então $t_{2}>t_{1}$.

Vejamos agora a definição de intersecção $\mathcal{F}$-transversal. Como antes, veremos primeiro o caso $M=\mathbb{R}^{2}$ e $\mathcal{F}$ sem singularidades. Note que $\phi_{z}$ denota a folha de $\mathcal{F}$ que passa por $z$.

Definição 3.14. Sejam $\gamma_{i}: J_{i} \rightarrow \mathbb{R}^{2}, i \in\{1,2\}$, dois caminhos transversos tais que $\phi_{\gamma_{1}\left(t_{1}\right)}=$ $\phi_{\gamma_{2}\left(t_{2}\right)}=\phi$. Diremos que $\gamma_{1}$ intersecta $\mathcal{F}$-transversalmente $\gamma_{2}$ em $\phi$ se existem $a_{1}, b_{1} \in J_{1}$ com $a_{1}<t_{1}<b_{1}$ e $a_{2}, b_{2} \in J_{2}$ com $a_{2}<t_{2}<b_{2}$ tais que

(i) $\phi_{\gamma_{2}\left(a_{2}\right)}$ está abaixo de $\phi_{\gamma_{1}\left(a_{1}\right)}$ com relação à $\phi$;

(ii) $\phi_{\gamma_{2}\left(b_{2}\right)}$ está acima de $\phi_{\gamma_{1}\left(b_{1}\right)}$ com relação à $\phi$.

Nesse caso, denotaremos $\left.\left.\gamma_{1}\right|_{\left[a_{1}, b_{1}\right]} \pitchfork_{\mathcal{F}} \gamma_{2}\right|_{\left[a_{2}, b_{2}\right]}$. 


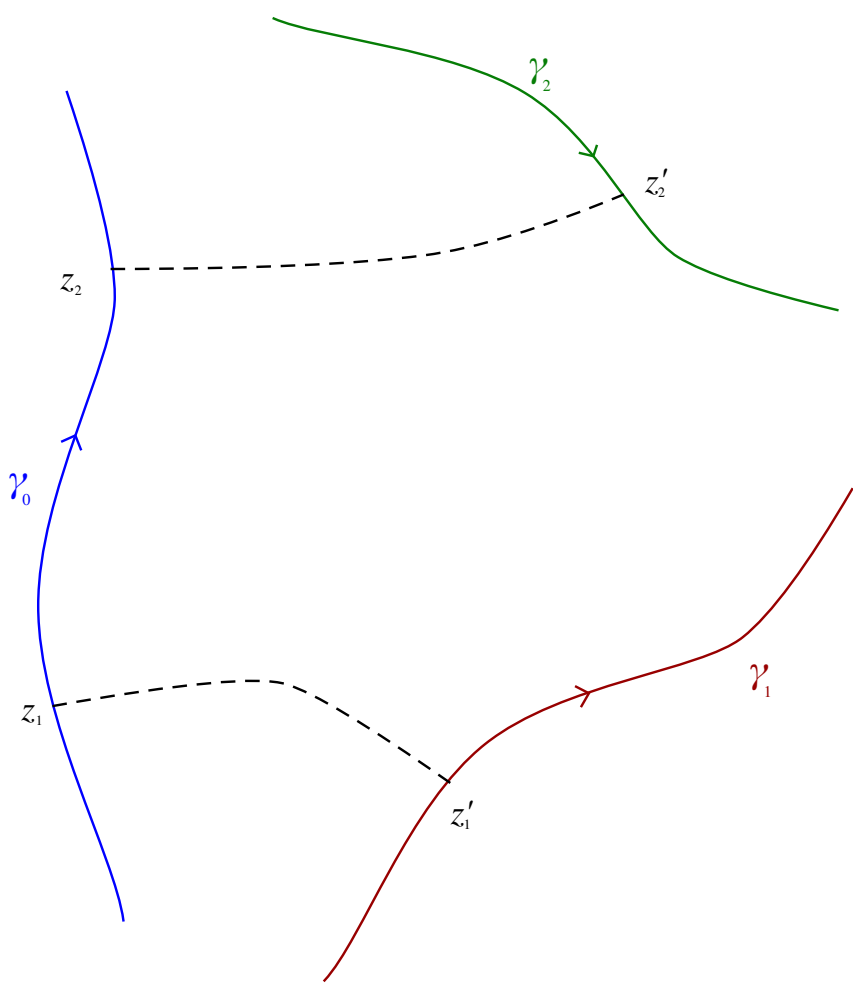

Figura 3.2: $\gamma_{2}$ acima de $\gamma_{1}$ com respeito à $\gamma_{0}$

No caso geral, diremos que dois caminhos transversos se intersectam $\mathcal{F}$-transversalmente se existem levantamentos dos caminhos e da folha onde ocorre a intersecção para o recobrimento universal, e tais levantamentos satisfazem a definição anterior. Quando não houver possibilidade de confusão, diremos simplesmente que dois caminhos transversos se intersectam transversalmente. No caso de um caminho $\gamma$ intersectar transversalmente a si mesmo, diremos que $\gamma$ posui uma autointersecção transversa.

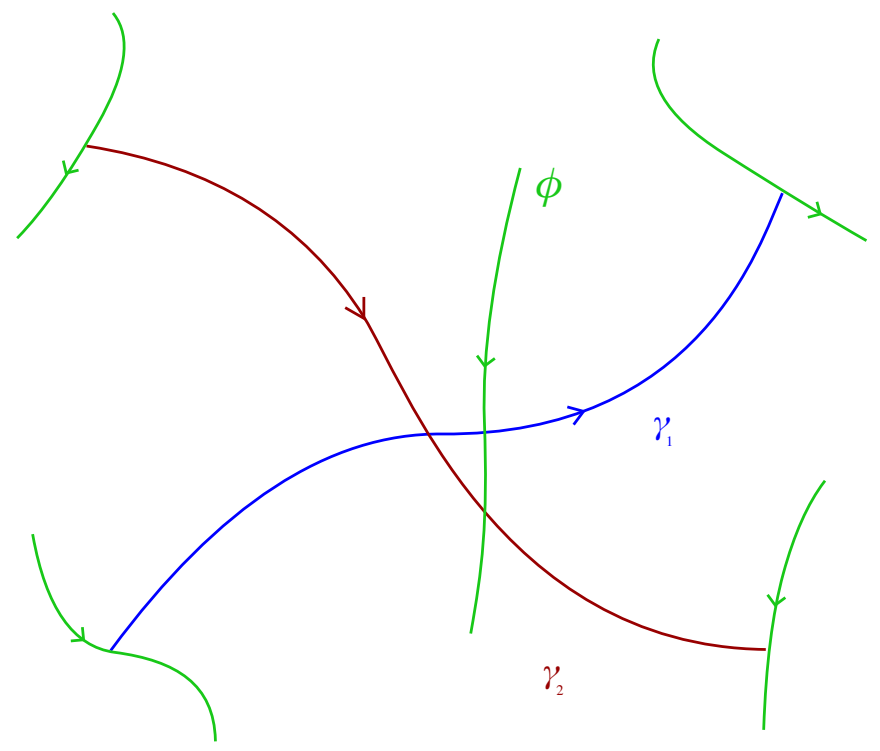

Figura 3.3: $\gamma_{1}$ e $\gamma_{2}$ com intersecção $\mathcal{F}$-transversa

Note que se $\left.\left.\gamma_{1}\right|_{I_{1}} \pitchfork_{\mathcal{F}} \gamma_{2}\right|_{I_{2}}$ e $\left.\left.\gamma_{2}\right|_{I_{2}} \sim \mathcal{F} \gamma_{3}\right|_{I_{3}}$, temos que $\left.\left.\gamma_{1}\right|_{I_{1}} \pitchfork_{\mathcal{F}} \gamma_{3}\right|_{I_{3}}$. Note ainda que se 
$\left.\left.\gamma_{1}\right|_{I_{1}} \pitchfork_{\mathcal{F}} \gamma_{2}\right|_{I_{2}}$ em $\phi$ não temos necessariamente que $\gamma_{1}$ e $\gamma_{2}$ se intersectam em um ponto de $\phi$. Contudo, $\left.\gamma_{1}\right|_{I_{1}}$ e $\left.\gamma_{2}\right|_{I_{2}}$ possuem ao menos um ponto de intersecção. Além disso, quando $M=\mathbb{R}^{2}$, é possível encontrar caminhos $\left.\gamma_{1}^{\prime} \sim_{\mathcal{F}} \gamma_{1}\right|_{I_{1}}$ e $\left.\gamma_{2}^{\prime} \sim_{\mathcal{F}} \gamma_{2}\right|_{I_{2}}$ tais que $\gamma_{1}^{\prime}$ e $\gamma_{2}^{\prime}$ possuem exatamente um ponto de intersecção e tal ponto está na folha $\phi$.

\section{$3.3 \quad$ Forcing}

Sejam agora $M$ uma superfície orientada, $f: M \rightarrow M$ um homeomorfismo isotópico à identidade, e denotemos por $\mathcal{I}$ o espaço das isotopias entre $f$ e a identidade, isto é, se $I \in \mathcal{I}$, temos que $I=\left(f_{t}\right)_{t \in[0,1]}$, onde $f_{0}=\operatorname{Id}_{M}, f_{1}=f$, para todo $t \in[0,1]$ temos que $f_{t}$ é homeomorfismo de $M$ e $I$ é uma curva contínua no espaço dos homeomorfismos de $M$, munido com a topologia da convergência uniforme em subconjuntos compactos. A trajetória de um ponto $z \in M$ é definida como o caminho $t \mapsto f_{t}(z)$, que denotaremos por $I(z)$. Usando concatenação de caminhos, podemos definir, para $n \in \mathbb{N}$,

$$
I^{n}(z)=\Pi_{0 \leq k<n} I\left(f^{k}(z)\right), \quad I^{\mathbb{N}}(z)=\Pi_{k \geq 0} I\left(f^{k}(z)\right) \text { e } I^{\mathbb{Z}}(z)=\Pi_{k \in \mathbb{Z}} I\left(f^{k}(z)\right) .
$$

Denotemos por fix $(I)=\bigcap_{t \in[0,1]}$ fix $\left(f_{t}\right)$, isto é, o conjunto dos pontos cujo caminho da isotopia é constante. Denotaremos por dom $(I)$ o complemento de fix $(I)$, tal conjuto será dito o domínio da isotopia $I$. Podemos definir uma pré-ordem em $\mathcal{I}$ da seguinte forma:

Definição 3.15. Sejam $I_{1}, I_{2} \in \mathcal{I}$. Diremos que $I_{1} \leqslant I_{2}$ se

(i) $\operatorname{fix}\left(I_{1}\right) \subset \operatorname{fix}\left(I_{2}\right)$;

(ii) $I_{2}$ é homotópico a $I_{1}$ relativo a fix $\left(I_{1}\right)$.

Diremos que que $I$ é uma isotopia maximal para a pré-ordem $\leqslant$ se para todo $z \in \operatorname{fix}(f) \backslash$ fix $(I)$, a trajetória de $z$ não é homotópica a zero em $\operatorname{dom}(I)$. Note que tal conceito é equivalente à maximalidade decorrente da pré-ordem $\leqslant$ definida acima.

Agora, note que se $I$ for uma isotopia maximal, denotando por $\tilde{I}=\left(\tilde{f}_{t}\right)_{t \in[0,1]}$ o levantamento de $\left.I\right|_{\operatorname{dom}(I)}$ para o recobrimento universal $\widetilde{\operatorname{dom}(I)}$, temos que $\tilde{f}_{1}=\tilde{f}$ é um homeomorfismo de Brouwer e $\tilde{f}_{0}=\operatorname{Id} \underset{\operatorname{dom}(I)}{ }$. Portanto, nesse caso, podemos obter um homeomorfismo de Brouwer a partir de um homeomorfismo de superfície, e assim obter uma folheação de Brouwer-Le Calvez para tal homeomorfismo que pode ser projetada na superfície original. Note que se simplesmente retirássemos todos os pontos fixos de $f$ da superfície poderíamos terminar com um homeomorfismo que não é isotópico à identidade, o que nos impediria de aplicar os resultados até aqui apresentados. Logo o conceito de isotopia maximal é a ferramenta fundamental na construção esboçada na Observação 3.8, portanto um questionamento natural é sob quais condições podemos obter uma isotopia maximal. O próximo resultado trata dessa questão.

Teorema 3.16 ([BCR16]). Para todo $I \in \mathcal{I}$, existe $I^{\prime} \in \mathcal{I}$ tal que $I \leqslant I^{\prime}$ e $I^{\prime}$ é maximal para a pré-ordem.

Vale notar que o Teorema 3.16 é uma extensão de resultados parcias de [Jau14], que por sua vez foram aplicados na construção apresentada em [CT18a], contudo o resultado de [BCR16], além de mais geral, simplifica significativamente a construção.

Portanto, aplicando o teorema anterior podemos sempre supor que temos em mãos uma isotopia maximal.

Usando este conceito de isotopia maximal e a existência da folheação de Brouwer-Le Calvez, é possível provar que dado $\tilde{z} \in \widehat{\operatorname{dom}(I)}$, onde $\tilde{z}$ denota um levantamento de $z$ para o recobrimento universal, existe um caminho transverso ligando $\tilde{z}$ a $\tilde{f}(\tilde{z})$. Mais precisamente: 
Teorema 3.17 ([Ca105]). Sendo I uma isotopia maximal, existe uma folheação topológica com singularidades $\mathcal{F}, \operatorname{com} \operatorname{dom}(\mathcal{F})=\operatorname{dom}(I)$ tal que para todo $z \in \operatorname{dom}(I)$ a trajetória $I(z)$ é homotópica em $\operatorname{dom}(I)$ com extremos fixos a um caminho transverso a $\mathcal{F}$, e esse caminho é único a menos de equivalência.

Denotaremos por $I_{\mathcal{F}}(z)$ a classe de caminhos transversos que ligam $z$ a $f(z)$ e que são homotópicos a $I(z)$ em $\operatorname{dom}(I)$ com extremos fixos. Denotaremos ainda

$$
I_{\mathcal{F}}^{n}(z)=\Pi_{0 \leq k<n} I_{\mathcal{F}}\left(f^{k}(z)\right), \quad I_{\mathcal{F}}^{\mathbb{N}}(z)=\Pi_{k \geq 0} I_{\mathcal{F}}\left(f^{k}(z)\right) \text { e } I_{\mathcal{F}}^{\mathbb{Z}}(z)=\Pi_{k \in \mathbb{Z}} I_{\mathcal{F}}\left(f^{k}(z)\right) .
$$

Definição 3.18. Diremos que um caminho transverso $\gamma:[a, b] \rightarrow \operatorname{dom}(\mathrm{I})$ é admissível de ordem $n$ se existe $z \in \operatorname{dom}(I)$ tal que $\gamma$ é equivalente a um caminho transverso da classe $I_{\mathcal{F}}^{n}(z)$.

Iremos apresentar agora o principal resultado da Teoria de Forcing, que nos permite obter novos caminhos admissíveis partindo de dois caminhos admissíveis que possuam intersecção transversa.

Proposição 3.19 ([CT18a]). Suponha que $\gamma_{i}:\left[a_{i}, b_{i}\right] \rightarrow M, i=1,2$ são dois caminhos transversos que se intersectam transversalmente em $\gamma_{1}\left(t_{1}\right)=\gamma_{2}\left(t_{2}\right)$. Se $\gamma_{1}$ é admissivel de ordem $n_{1}$ e $\gamma_{2}$ é admissivel de ordem $n_{2}$, então temos que $\left.\left.\gamma_{1}\right|_{\left[a_{1}, t_{1}\right]} \gamma_{2}\right|_{\left[t_{2}, b_{2}\right]}$ e $\left.\left.\gamma_{2}\right|_{\left[a_{2}, t_{2}\right]} \gamma_{1}\right|_{\left[t_{1}, b_{1}\right]}$ são admissíveis de ordem $n_{1}+n_{2}$. Além disso, ou um dos caminhos é admissivel de ordem $\min \left(n_{1}, n_{2}\right)$ ou ambos são admissiveis de ordem $\max \left(n_{1}, n_{2}\right)$.

O próximo resultado nos dará uma forma prática de demonstrar que dois caminhos possuem intersecção transversa, quando um dos caminhos é uma linha. Mas antes, vamos introduzir alguns conceitos necessários para tal proposição.

Definição 3.20. Sejam $\gamma: \mathbb{R} \rightarrow \mathbb{R}^{2}$ um caminho próprio e $\rho \in \mathbb{R}^{2}$, com $\|\rho\|=1$. Diremos que $\gamma$ é dirigido por $\rho$ se

$$
\lim _{t \rightarrow \pm \infty}\|\gamma(t)\|=+\infty, \quad \lim _{t \rightarrow+\infty} \gamma(t) /\|\gamma(t)\|=\rho, \quad \lim _{t \rightarrow-\infty} \gamma(t) /\|\gamma(t)\|=-\rho
$$

Note que quando $f: \mathbb{T}^{2} \rightarrow \mathbb{T}^{2}$ é um homeomorfismo homotópico à identidade e $z \in \mathbb{T}^{2}$ é um ponto com vetor de rotação não nulo $\rho$, então podemos encontrar $\gamma$ um representante de $I_{\mathcal{F}}^{\mathbb{Z}}(z)$, tal que todo levantamento de $\gamma$ para $\mathbb{R}^{2}$ é um caminho dirigido por $\rho /\|\rho\|$.

Definição 3.21. Seja $\gamma: \mathbb{R} \rightarrow \mathbb{R}^{2}$ uma linha (no sentido da Definição 3.2) transversa. Denotaremos as duas componente conexas de $\mathbb{R}^{2} \backslash[\gamma]$ por $R(\gamma)$ e $L(\gamma)$, denominadas a direita e a esquerda de $\gamma$, respectivamente, como na seção anterior. Além disso, podemos definir a direita folheada de $\gamma$, denotada por $r(\gamma)$, como o conjunto das folhas e singularidades de $\mathcal{F}$ contidas em $R(\gamma)$ (e analogamente a esquerda folheada de $\gamma$, denotada por $l(\gamma))$.

Portanto $l(\gamma) \cup r(\gamma)$ contém todas as folhas e singularidades que não intersectam $\gamma$ (ou, equivalentemente, seu complementar contém todas as folhas intersectadas por $\gamma$ ).

Proposição 3.22 ([CT]). Sejam $\mathcal{F}$ uma folheação topólogica orientada com singularidades de $\mathbb{R}^{2}$, $\gamma: \mathbb{R} \rightarrow \mathbb{R}^{2}$ um caminho transverso, sendo $\gamma$ uma linha ou um caminho próprio dirigido por um vetor unitário $\rho$, e $\gamma^{\prime}:[a, b] \rightarrow \mathbb{R}^{2}$ um caminho transverso. Se $\left[\gamma^{\prime}\right] \cap l(\gamma) \neq \emptyset,\left[\gamma^{\prime}\right] \cap r(\gamma) \neq \emptyset$ e se $I^{*}=\left\{t \in \mathbb{R} \mid \phi_{\gamma(t)}\right.$ cruza $\left.\gamma^{\prime}\right\}$ é limitado, então existem intervalos $I$ e $I^{\prime}$ tais que $\left.\left.\gamma^{\prime}\right|_{I^{\prime}} \pitchfork_{\mathcal{F}} \gamma\right|_{I}$.

O próximo resultado nos fornece uma espécie de "estabilidade" para a folheção, portanto podemos fazer pequenas perturbações em um ponto sem grandes mudanças nos caminhos transversos admissíveis associados. Mais precisamente, temos o seguinte:

Lema 3.23 ([CT18a]). Fixemos $z \in \operatorname{dom}(I), n \geq 1$, e parametrizemos $I_{\mathcal{F}}^{n}(z)$ por $[0,1]$. Para cada $0<a<b<1$, existe uma vizinhança $V$ de $z$ tal que, para todo $z^{\prime} \in V$, o caminho $\left.I_{\mathcal{F}}^{n}(z)\right|_{[a, b]}$ é $\mathcal{F}$-equivalente a um subcaminho de $I_{\mathcal{F}}^{n}\left(z^{\prime}\right)$. Além disso, existe uma vizinhança $W$ de $z$ tal que, para todos $z^{\prime}, z^{\prime \prime} \in W$, o caminho $I_{\mathcal{F}}^{n}\left(z^{\prime}\right)$ é $\mathcal{F}$-equivalente a um subcaminho de $I_{\mathcal{F}}^{n+2}\left(f^{-1}\left(z^{\prime \prime}\right)\right)$. 
Na demonstração do resultado principal deste trabalho, uma propriedade importante que usaremos com constância é o fato que pontos típicos de medidas ergódicas são recorrentes (um ponto é dito recorrente quando existe uma subsequência de sua órbita que converge para tal ponto). Vamos apresentar agora uma definição análoga para caminhos transversos.

Definição 3.24. Um caminho transverso $\gamma: \mathbb{R} \rightarrow M$ será chamado $\mathcal{F}$-recorrente se para todo segmento compacto $J \subset \mathbb{R}$ e todo $t \in \mathbb{R}$ existirem segmentos $J^{\prime} \subset(-\infty, t]$ e $J^{\prime \prime} \subset[t,+\infty)$ tais que $\left.\left.\gamma\right|_{J^{\prime}} \sim_{\mathcal{F}} \gamma\right|_{J}$ e $\left.\left.\gamma\right|_{J^{\prime \prime}} \sim_{\mathcal{F}} \gamma\right|_{J}$

Uma importante consequência do Lema 3.23 é a seguinte, que conecta os conceitos de pontos recorrentes e caminhos transversos recorrentes.

Corolário 3.25 ([CT18a]). Se $z \in \operatorname{dom}(I)$ é um ponto recorrente de $f$, então $I_{\mathcal{F}}^{\mathbb{Z}}(z)$ é $\mathcal{F}$-recorrente.

A próxima proposição técnica também nos será útil na demonstração do resultado principal do trabalho.

Proposição 3.26 ([CT18a]). Sejam $f: \mathbb{T}^{2} \rightarrow \mathbb{T}^{2}$ um homeomorfismo isotópico à identidade, $\tilde{f}: \mathbb{R}^{2} \rightarrow \mathbb{R}^{2}$ um levantamento de $f$ e suponhamos que $\rho(\tilde{f})=\left\{t \rho_{0} \mid 0 \leq t \leq 1\right\}$, onde $\tan \left(\rho_{0}\right) \notin \mathbb{Q}$. Então existe um ponto $\tilde{y}_{0} \in \operatorname{dom}(\tilde{\mathcal{F}})$ tal que para todo $\epsilon \in\{-1,1\}$ existe uma sequência $\left(p_{l}, q_{l}\right)_{l \geq 0}$ em $\mathbb{Z}^{2} \times \mathbb{N}$ satisfazendo:

$$
\lim _{l \rightarrow+\infty} q_{l}=+\infty, \quad \lim _{l \rightarrow+\infty} \tilde{f}^{q_{l}}\left(\tilde{y}_{0}\right)-\tilde{y}_{0}-p_{l}=0, \quad \epsilon\left\langle p_{l}, \rho_{0}^{\perp}\right\rangle>0
$$

e existe uma sequência $\left(p_{l}^{\prime}, q_{l}^{\prime}\right)_{l \geq 0}$ em $\mathbb{Z}^{2} \times \mathbb{N}$ satisfazendo:

$$
\lim _{l \rightarrow+\infty} q_{l}^{\prime}=+\infty, \quad \lim _{l \rightarrow+\infty} \tilde{f}^{-q_{l}^{\prime}}\left(\tilde{y}_{0}\right)-\tilde{y}_{0}-p_{l}^{\prime}=0, \quad \epsilon\left\langle p_{l}^{\prime}, \rho_{0}^{\perp}\right\rangle>0 .
$$

Observação 3.27. Note que pela construção feita na demonstração da Proposição anterior (o Lema 77 em [CT18a]), é possível obter que $\rho\left(\tilde{f}, y_{0}\right)=\rho_{0}$, onde $y_{0}=\pi\left(\tilde{y}_{0}\right)$

O próximo teorema é um importante resultado sobre a realização de vetores de rotação, que nos permite relacionar os conceitos de forcing e teoria de rotação.

Teorema 3.28 ([CT18b]). Seja $M$ uma superfície orientada, $f$ um homeomorfismo de $M$ isotópico à identidade, I uma isotopia maximal de $f$ e $\mathcal{F}$ uma folheação transersa à $I$. Suponha que $\gamma:[a, b] \rightarrow$ $\operatorname{dom}(I)$ é um caminho admissivel de ordem $r$ com uma autointersecção transversa em $\gamma(s)=\gamma(t)$, onde $s<t$. Sejam $\tilde{\gamma}$ um levantamento de $\gamma$ para o recobrimento universal $\widetilde{\operatorname{dom}}(I)$ de $\operatorname{dom}(I) e$ $T$ uma aplicação de recobrimento tais que $\tilde{\gamma}$ e $T(\tilde{\gamma})$ possuem uma interseç̧ão $\tilde{\mathcal{F}}$-transversa em $\tilde{\gamma}(t)=T(\tilde{\gamma})(s)$. Seja $\tilde{f}$ o levantamento de $\left.f\right|_{\operatorname{dom}(I)}$ para $\widetilde{\operatorname{dom}}(I)$, e $\hat{f}$ o homeomorfismo do espaço de recobrimento anular $\widehat{\operatorname{dom}}(I)=\widetilde{\operatorname{dom}}(I) / T$ levantado por $\tilde{f}$. Temos então o seguinte:

(i) Para todo número racional $p / q \in(0,1]$, escrito em forma irredutivel, existe um ponto $\tilde{z} \in$ $\widetilde{\operatorname{dom}}(I)$ tal que $\tilde{f}^{q r}(\tilde{z})=T^{p}(\tilde{z})$ e $\tilde{I}_{\tilde{\mathcal{F}}}^{\mathbb{Z}}(\tilde{z})$ é equivalente a $\Pi_{k \in \mathbb{Z}} T^{k}\left(\left.\tilde{\gamma}\right|_{[s, t]}\right)$;

(ii) Para todo número irracional $\lambda \in[0,1 / r]$, existe um conjunto compacto $\hat{Z}_{\rho} \subset \widehat{\operatorname{dom}}(I)$ invariante por $\hat{f}$, tal que todo ponto $\hat{z} \in \hat{Z}_{\rho}$ possui número de rotação $\rho(\tilde{f}, \tilde{z})=\lambda$. Além disso, se $\tilde{z} \in \widetilde{\operatorname{dom}}(I)$ é um levantamento de $\hat{z}$, então $\tilde{I}_{\tilde{\mathcal{F}}}^{\mathbb{Z}}(\tilde{z})$ é equivalente a $\Pi_{k \in \mathbb{Z}} T^{k}\left(\left.\tilde{\gamma}\right|_{[s, t]}\right)$. 


\section{Capítulo 4}

\section{Difusão sublinear}

\subsection{Enunciado do problema}

Seja $f: \mathbb{T}^{2} \rightarrow \mathbb{T}^{2}$ um homeomorfismo do toro isotópico à identidade, $\tilde{f}: \mathbb{R}^{2} \rightarrow \mathbb{R}^{2}$ um levantamento de $f$ e $\pi: \mathbb{R}^{2} \rightarrow \mathbb{T}^{2}$ a aplicação de recobrimento.

Suponhamos que $\rho(\tilde{f})=\left\{t \rho_{0} \mid 0 \leq t \leq 1\right\}$, onde $\tan \left(\rho_{0}\right) \notin \mathbb{Q}$. Denotando por $(x)_{1}$ e $(x)_{2}$ a primeira e segunda coordenadas, respectivamente, de um ponto $x \in \mathbb{R}^{2}$, se $\rho_{0}=\left(\left(\rho_{0}\right)_{1},\left(\rho_{0}\right)_{2}\right)$, denotaremos $\rho_{0}^{\perp}=\left(-\left(\rho_{0}\right)_{2},\left(\rho_{0}\right)_{1}\right)$.

Queremos provar o seguinte:

Teorema A. Nas condiçôes acima, existe $M>0$ tal que

$$
\left|\left\langle\tilde{f}^{n}(\tilde{z})-\tilde{z}, \rho_{0}^{\perp}\right\rangle\right|<M,
$$

para todo $\tilde{z} \in \mathbb{R}^{2}$ e $n \in \mathbb{Z}$.

\subsection{Demonstração do Teorema A}

Fixemos uma isotopia $I: \mathbb{T}^{2} \times[0,1] \rightarrow \mathbb{T}^{2}$ entre $f$ e a identidade, tal que fix $(I) \neq \emptyset$, e $\tilde{I}$ : $\mathbb{R}^{2} \times[0,1] \rightarrow \mathbb{R}^{2}$ o levantamento de $I$ tal que fix $(\tilde{I}) \neq \emptyset$. Pelo Teorema 3.16, podemos assumir que $I$ é maximal. Seja $\mathcal{F}$ a folheação de $\mathbb{T}^{2}$ dada pelo Teorema 3.17 , e $\tilde{\mathcal{F}}$ seu levantamento para $\mathbb{R}^{2}$. Seja também $\mu_{0}$ uma medida ergódica associada ao vetor de rotação $\rho_{0}$, e fixemos $z_{0}$ um ponto típico de $\mu_{0}$ e $\tilde{z}_{0}$ um levantamento de $z_{0}$. Como $\mu_{0}$ é ergódica, podemos assumir que $z_{0}$ é recorrente. Suponhamos ainda que $\rho_{0}$ está no primeiro quadrante (os outros casos são análogos).

Lema 4.1. Existem linhas $\tilde{\mathcal{F}}$-transversas $\alpha_{-}, \alpha_{+}: \mathbb{R} \rightarrow \mathbb{R}^{2}$ e $v_{-}, v_{+} \in \mathbb{Z}^{2}$ tais que $\alpha_{-}(t+1)=$ $\alpha_{-}(t)+v_{-}, \alpha_{+}(t+1)=\alpha_{+}(t)+v_{+}$, para todo $t \in \mathbb{R},\left\langle v_{-}, \rho_{0}^{\perp}\right\rangle\left\langle 0,\left\langle v_{-}, \rho_{0}\right\rangle>0,\left\langle v_{+}, \rho_{0}^{\perp}\right\rangle>0 e\right.$ $\left\langle v_{+}, \rho_{0}\right\rangle>0$.

Demonstração. Vamos começar construindo $\alpha_{+}$e $v_{+}$. Sejam $\tilde{y}_{0}$ e $\left(p_{l}, q_{l}\right)_{l \geq 0}$ dados pela Proposição 3.26 tais que $\lim _{l \rightarrow+\infty} q_{l}=+\infty, \lim _{l \rightarrow+\infty}\left(\tilde{f}^{q_{l}}\left(\tilde{y}_{0}\right)-\tilde{y}_{0}-p_{l}\right)=0$ e $\left\langle p_{l}, \rho_{0}^{\perp}\right\rangle>0$. Fixemos $\alpha_{0} \in \tilde{I}_{\tilde{\mathcal{F}}}\left(\tilde{y}_{0}\right)$. Sejam $V_{0} \subset \mathbb{R}^{2}$ uma vizinhança tubular de $\tilde{y}_{0}$ e $h_{0}: V_{0} \rightarrow(0,1)^{2}$ um homeomorfismo que mapeia a folheação em $V_{0}$ na folheação vertical orientada para baixo em $(0,1)^{2}$ tais que $\left(h_{0}\left(\left[\alpha_{0}\right] \cap V_{0}\right)\right)_{1}=$ $\left[\left(h_{0}\left(\tilde{y}_{0}\right)\right)_{1}, 1\right)$ (isto é, $h\left(\alpha_{0} \cap V_{0}\right)$ cruza todas as folhas em $(0,1)^{2}$ à direita da folha que passa por $\left.h_{0}\left(\tilde{y}_{0}\right)\right)$. Seja também $\varepsilon>0$ tal que $B\left(\varepsilon, \tilde{y}_{0}\right) \subset V_{0}$. Pela Observação 3.27 temos que $\rho\left(\tilde{f}, \tilde{y}_{0}\right)=$ $\rho_{0}$, portanto existe $l_{0} \in \mathbb{N}$ tal que para $l \geq l_{0}$ temos $\left\langle p_{l}, \rho_{0}\right\rangle>0$. Pela Proposição 3.26 temos $\lim _{l \rightarrow+\infty}\left(\tilde{f}^{q_{l}}\left(\tilde{y}_{0}\right)-\tilde{y}_{0}-p_{l}\right)=0$, assim existe $l_{1}>0$ tal que $\tilde{f}^{q_{l}}\left(\tilde{y}_{0}\right)-p_{l} \in B\left(\varepsilon, \tilde{y}_{0}\right)$ e $\left\langle p_{l}, \rho_{0}^{\perp}\right\rangle>0$, se $l \geq l_{1}$. Sendo $l^{\prime}=\max \left\{l_{0}, l_{1}\right\}$, vamos denotar $N=q_{l^{\prime}}$ e $v_{+}=p_{l^{\prime}}$.

Consideremos agora $\alpha_{N} \in \tilde{I}_{\tilde{\mathcal{F}}}^{N}\left(\tilde{y}_{0}\right)$ tal que $\left[\alpha_{0}\right] \subset\left[\alpha_{N}\right]$ e $\alpha_{N}$ é parametrizada por $[0,1]$. Temos que, como $\tilde{f}^{N}\left(\tilde{y}_{0}\right)-v_{+} \in B\left(\varepsilon, \tilde{y}_{0}\right), \tilde{f}^{N}\left(\tilde{y}_{0}\right)-v_{+} \in V_{0}$, portanto podemos modificar $\alpha_{N}$ em $V_{0}$ de forma a obter um caminho transverso $\alpha_{N}^{\prime}:[0,1] \rightarrow \mathbb{R}^{2}$ de forma que $\alpha_{N}$ e $\alpha_{N}^{\prime}$ coincidem fora de 


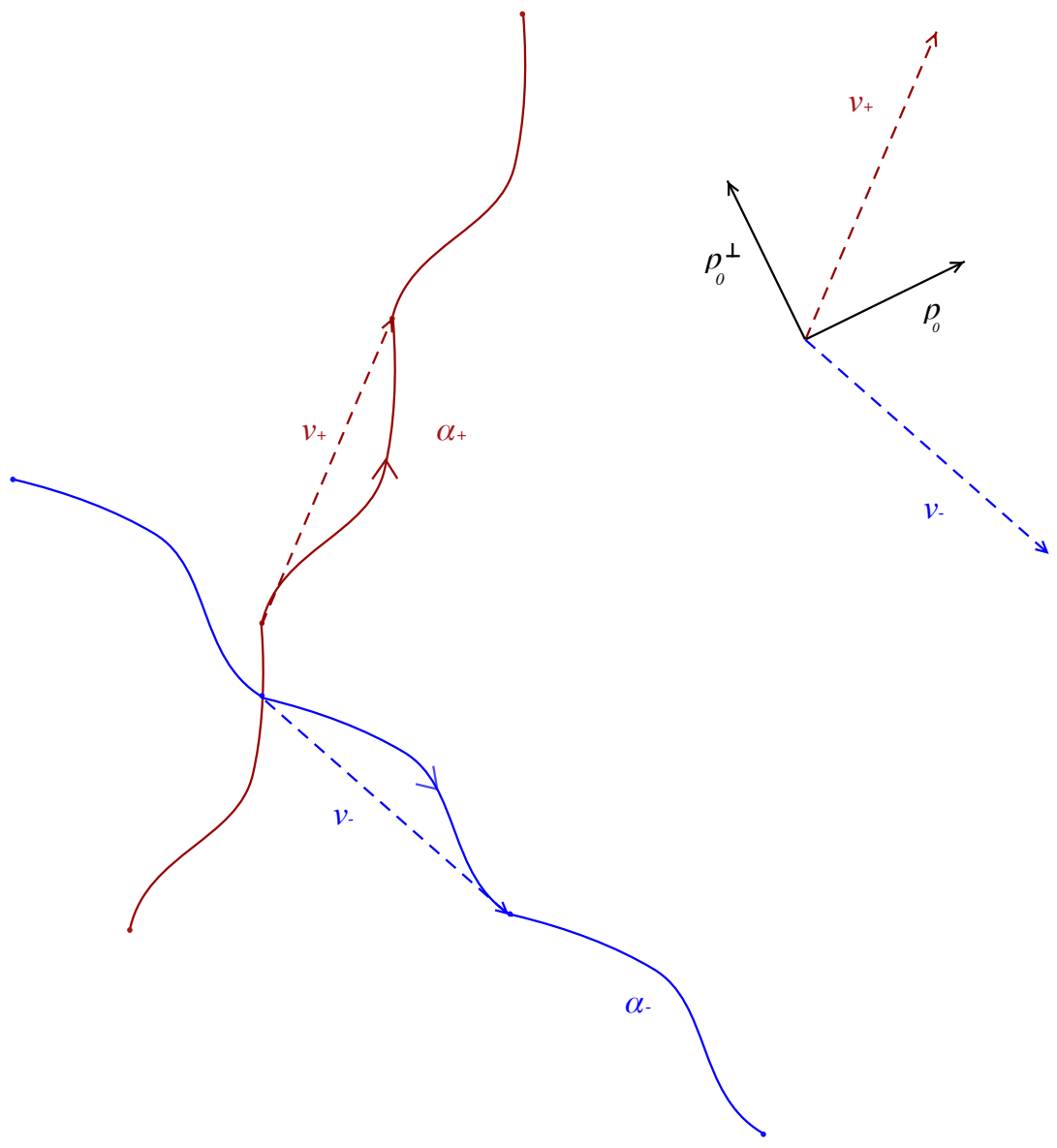

Figura 4.1: Ilustração do Lema 4.1

$V_{0}$ e $\alpha_{N}^{\prime}(0)=\tilde{f}^{N}\left(\tilde{y}_{0}\right)-v_{+}$(para modificar $\alpha_{N}$ em $V_{0}$, basta modificar $h_{0}\left(\alpha_{N} \cap V_{0}\right)$ em $(0,1)^{2}$ e levar de volta para $V_{0}$ usando $h_{0}^{-1}$, veja a Figura 4.2). Agora, definamos $\alpha_{+}^{\prime}=\Pi_{k \in \mathbb{Z}}\left(\alpha_{N}^{\prime}+k v_{+}\right)$. Como $\alpha_{+}^{\prime}$ é um caminho transverso, se $\alpha_{+}^{\prime}$ possui autointersecção, isto é, se $\alpha_{+}^{\prime}(s)=\alpha_{+}^{\prime}(t)$, com $s<t$, podemos remover o trecho $\left.\alpha_{+}^{\prime}\right|_{(s, t]}$ e reparametrizar da forma adequada, obtendo assim um novo caminho, que denotaramos por $\alpha_{+}$. Assim podemos assumir que $\alpha_{+}$é um caminho simples e portanto, pela construção feita, temos que $\alpha_{+}$é uma linha que satisfaz as condições pedidas.

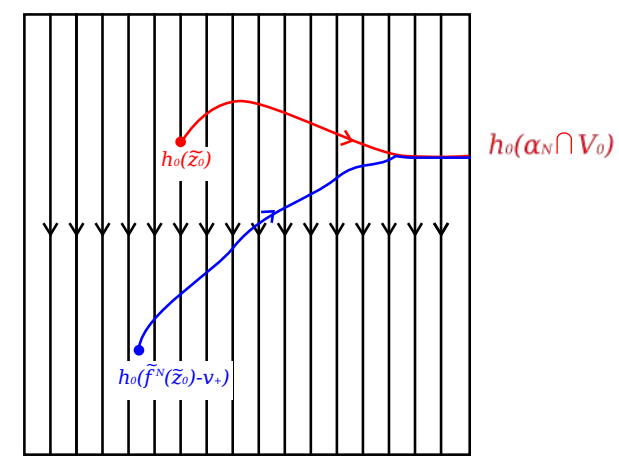

Figura 4.2: Construção de $\alpha_{N}^{\prime}$

A construção de $\alpha_{-}$e $v_{-}$é análoga, usando a Proposição 3.26 com $\epsilon=-1$.

Lema 4.2. Existe $L_{0}>0$ tal que, para todo $\tilde{x} \in \mathbb{R}^{2} \backslash \operatorname{sing}(\tilde{\mathcal{F}})$ existe um caminho transverso $\tilde{\gamma}_{\tilde{x}} \in \tilde{I}_{\tilde{\mathcal{F}}}(\tilde{x})$, tal que $\operatorname{diam}\left(\tilde{\gamma}_{\tilde{x}}\right)<L_{0}$. 
Demonstração. Primeiramente, notemos que basta provar o resultado para pontos em $[0,1]^{2}$, já que $\tilde{I}_{\tilde{\mathcal{F}}}(\tilde{x}+w)=\tilde{I}_{\tilde{\mathcal{F}}}(\tilde{x})+w$ para todo $\tilde{x} \in \mathbb{R}^{2}$ e todo $w \in \mathbb{Z}^{2}$. Agora, pela continuidade da isotopia $\tilde{I}$, temos que existe $L>0$ tal que $\tilde{I}\left([0,1]^{2} \times[0,1]\right) \subset B(L, 0)$, ou seja, para todo ponto $\tilde{x} \in[0,1]^{2}$ o caminho da isotopia $\tilde{I}(\tilde{x})$ está contido em $B(L, 0)$.

Sejam agora as linhas transversas $\alpha_{+}$e $\alpha_{-}$dados pelo Lema 4.1, e sejam também $v_{1}, v_{2}, v_{3}, v_{4} \in$ $\mathbb{Z}^{2}$ de forma que $B(L, 0)$ está contido em uma componente conexa limitada de $\mathbb{R}^{2} \backslash\left(\left[\alpha_{+}+v_{1}\right] \cup\right.$ $\left.\left[\alpha_{-}+v_{2}\right] \cup\left[\alpha_{+}+v_{3}\right] \cup\left[\alpha_{-}+v_{4}\right]\right)$, que denotaremos por $U$, e $U \subset R\left(\alpha_{+}+v_{1}\right) \cap R\left(\alpha_{-}+v_{2}\right) \cap L\left(\alpha_{+}+\right.$ $\left.v_{3}\right) \cap L\left(\alpha_{-}+v_{4}\right)$ (veja a Figura 4.3).

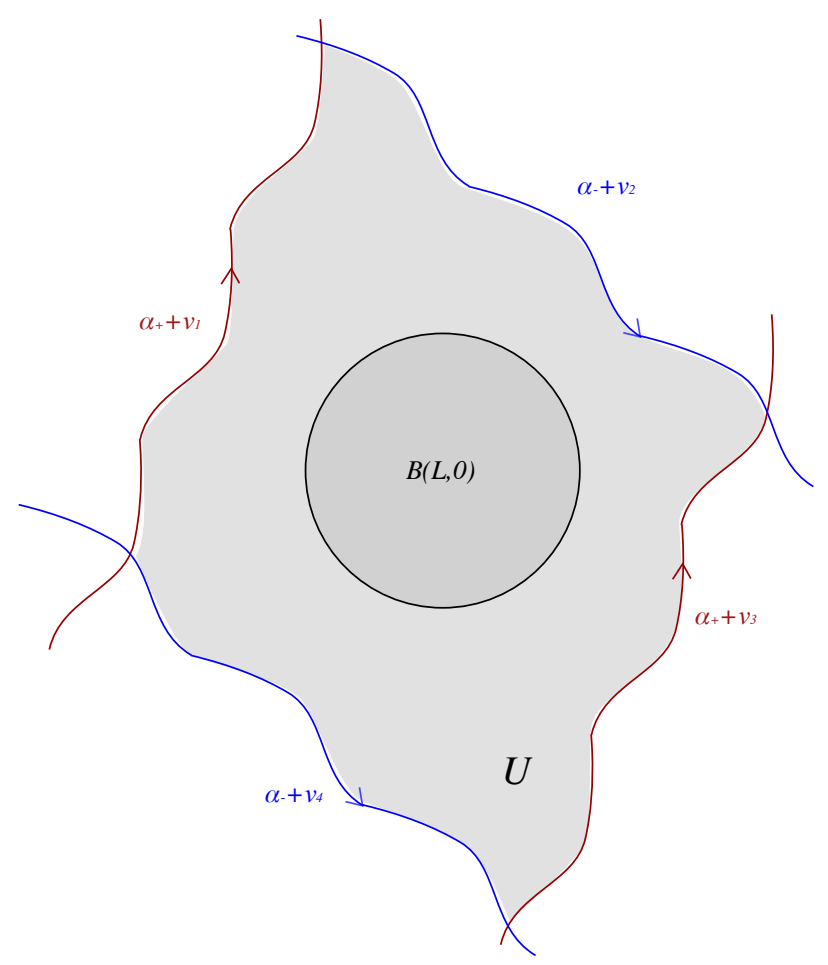

Figura 4.3: Ilustração do conjunto $U$

Afirmamos que se $\phi$ é uma folha de $\tilde{\mathcal{F}}$ que intersecta $U$, então $[\phi] \cap U$ é conexo (ou seja, um segmento de $\phi$ ). De fato, pela Observação 3.12, como $\alpha_{+}$e $\alpha_{-}$(bem como seus transladados) são linhas transversas, temos que a folha $\phi$ cruza cada linha no máximo uma vez, e sempre da esquerda para a direita da linha. Sejam agora $t_{1}<t_{2}$ tais que $\phi\left(t_{i}\right) \in U$. Note que para provarmos que $[\phi] \cap U$ é conexo basta provarmos que $\phi(t) \in U$, para todo $t_{1}<t<t_{2}$. Como $\phi\left(t_{1}\right) \in U$ e $t>t_{1}$, temos que $\phi(t) \in R\left(\alpha_{+}+v_{1}\right)$ e $\phi(t) \in R\left(\alpha_{-}+v_{2}\right)$. Analogamente, como $\phi\left(t_{2}\right) \in U$ e $t<t_{2}$, temos que $\phi(t) \in L\left(\alpha_{+}+v_{3}\right)$ e $\phi(t) \in L\left(\alpha_{-}+v_{4}\right)$. Assim, $\phi(t) \in R\left(\alpha_{+}+v_{1}\right) \cap R\left(\alpha_{-}+v_{2}\right) \cap L\left(\alpha_{+}+\right.$ $\left.v_{3}\right) \cap L\left(\alpha_{-}+v_{4}\right)$. Além disso, como $\phi\left(t_{1}\right)$ e $\phi\left(t_{2}\right)$ estão em $U$, temos que nenhum ponto de $\left.\phi\right|_{\left[t_{1}, t_{2}\right]}$ intersecta $\left[\alpha_{+}+v_{1}\right] \cup\left[\alpha_{-}+v_{2}\right] \cup\left[\alpha_{+}+v_{3}\right] \cup\left[\alpha_{-}+v_{4}\right]$. Portanto $\phi(t) \in U$, provando assim que $[\phi] \cap U$ é conexo.

Sejam $\widehat{\operatorname{dom}}(\tilde{I})$ o recobrimento universal de $\operatorname{dom}(\tilde{I}), \hat{\pi}: \widehat{\operatorname{dom}}(\tilde{I}) \rightarrow \operatorname{dom}(\tilde{I})$ a aplicação de recobrimento, $\widehat{I}$ um levantamento de $\left.\tilde{I}\right|_{\operatorname{dom}(\tilde{I})}$ para $\widehat{\operatorname{dom}}(\tilde{I})$ e $\hat{\mathcal{F}}$ o levantamento da folheação $\tilde{\mathcal{F}}$ para $\widehat{\operatorname{dom}}(\tilde{I})$. Como $I$ é maximal, temos que $\tilde{I}$ é maximal, e portanto $\hat{f}: \widehat{\operatorname{dom}}(\tilde{I}) \rightarrow \widehat{\operatorname{dom}}(\tilde{I})$ é um homeomorfismo de Brouwer, onde $\hat{f}$ é o levantamento natural de $\left.\tilde{f}\right|_{\operatorname{dom}(\tilde{I})}$. Fixemos agora $\tilde{x} \in[0,1]^{2}$ tal que $\tilde{x} \notin \operatorname{sing}(\tilde{\mathcal{F}})$ e $\hat{x} \in \widehat{\operatorname{dom}}(\tilde{I})$ um levantamento de $\tilde{x}$. Sendo $\Phi(\hat{x})=\{\phi \in \hat{\mathcal{F}} \mid \hat{x} \in R(\phi)$ e $\hat{f}(\hat{x}) \in$ $L(\phi)\} \cup\left\{\phi_{\hat{x}}, \phi_{\hat{f}(\hat{x})}\right\}$, note que $\Phi(\hat{x})$ é o conjunto das folhas que intersectam a trajetória transversa $\hat{I}_{\hat{\mathcal{F}}}(\hat{x})$, e além disso, $\Phi(\hat{x})$ é totalmente ordenado pela relação $\phi_{1}<\phi_{2}$ se $R\left(\phi_{1}\right) \subset R\left(\phi_{2}\right)$, pois $\hat{f}$ é um homeomorfismo de Brouwer e as folhas de $\hat{\mathcal{F}}$ são linhas de Brouwer. Usando tal ordem, podemos parametrizar $\Phi(\hat{x})$ por um parâmetro $s \in[0,1]$ de forma que $\phi_{0}=\phi_{\hat{x}}$ e $\phi_{1}=\phi_{\hat{f}(\hat{x})}$. 
Denotando o caminho da isotopia $\hat{I}(\hat{x}):[0,1] \rightarrow \widehat{\operatorname{dom}}(\tilde{I})$ por $\hat{\beta}$, podemos definir as seguintes funções do parâmetro $s$

$$
t_{-}(s)=\left\{\begin{array}{l}
0, \text { se } s=0 \\
\inf \left\{t \in[0,1] \mid \hat{\beta}([t, 1]) \cap R\left(\phi_{s}\right)=\emptyset\right\}, \text { se } s \in(0,1]
\end{array}\right.
$$

e

$$
t_{+}(s)=\left\{\begin{array}{l}
\inf \left\{t \in[0,1] \mid \hat{\beta}([t, 1]) \subset L\left(\phi_{s}\right)\right\}, \text { se } s \in[0,1) \\
1, \text { se } s=1 .
\end{array}\right.
$$

Intuitivamente, $t_{-}(s)$ é o instante que $\hat{\beta}$ esteve do lado direito de $\phi_{s}$ pela última vez, e $t_{+}(s)$ é o primeiro instante em que $\hat{\beta}$ fica sempre do lado esquerdo de $\phi_{s}$. Note que, se $s_{1}<s_{2}$, então $t_{-}\left(s_{1}\right) \leq t_{+}\left(s_{1}\right)<t_{-}\left(s_{2}\right) \leq t_{+}\left(s_{2}\right)$. Portanto temos que $t_{-}$e $t_{+}$coincidem, exceto possivelmente num conjunto enumerável de descontinuidades. Contudo, note ainda que se enumerarmos os pontos de descontinuidade como $\left(s_{i}\right)_{i \in \mathbb{N}}$, temos que $\sum d_{i} \leq 1$, onde $d_{i}=t_{+}\left(s_{i}\right)-t_{-}\left(s_{i}\right)\left(\right.$ pois $t_{ \pm}([0,1]) \subset$ $[0,1])$.

Agora vamos definir um caminho $\gamma_{\hat{x}}^{*}:[0,1] \rightarrow \widehat{\operatorname{dom}}(\tilde{I})$ que será transverso exceto nas descontinuidades de $t_{-}$e $t_{+}$da seguinte forma: fazemos $\gamma_{\hat{x}}^{*}$ ser igual a $\hat{\beta}$ nos pontos onde $t_{-}(s)=t_{+}(s)$ e nos pontos onde $t_{-}(s) \neq t_{+}(s)$ fazemos $\gamma_{\hat{x}}^{*}$ ser igual ao segmento da folha $\phi_{s}$ que liga os pontos $\hat{\beta}\left(t_{-}(s)\right)$ e $\hat{\beta}\left(t_{+}(s)\right)$ (veja a Figura 4.4).
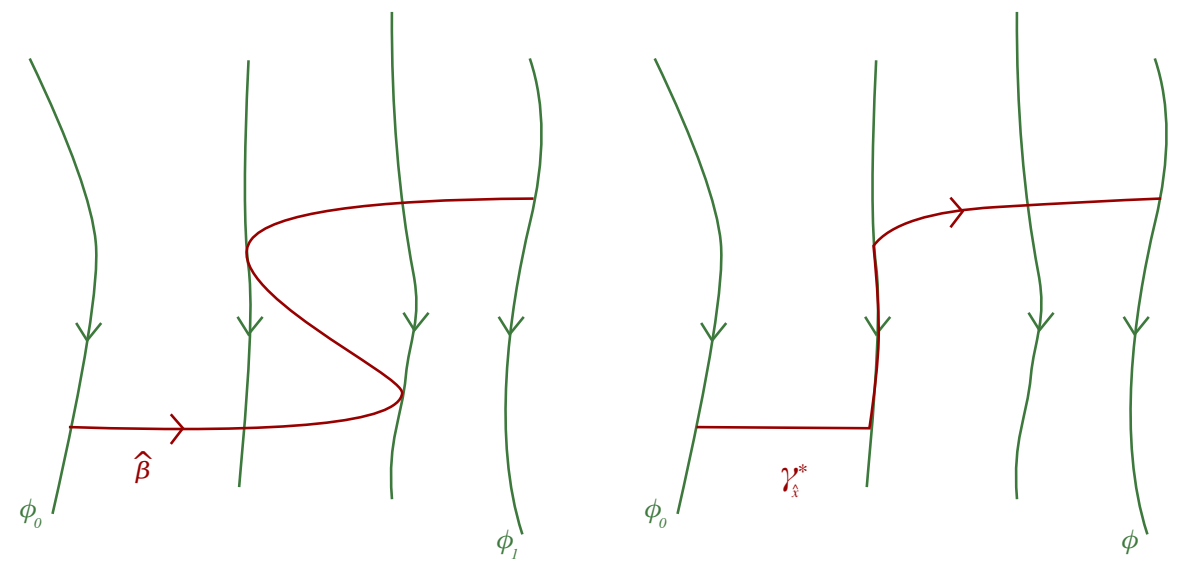

Figura 4.4: Ilustração das curvas $\hat{\beta}$ e $\gamma_{\hat{x}}^{*}$

Agora, como $\gamma_{\hat{x}}^{*}$ é composto por pontos de folhas ou segmentos de folhas, temos que para cada $s \in[0,1]$ podemos encontrar $V_{s}$ uma vizinhança tubular do ponto (ou segmento) de $\gamma_{\hat{x}}^{*}$ que intersecta a folha $\phi_{s}$ e $\varepsilon_{s} \in(0,1)$ de forma que $V_{s} \subset B\left(\varepsilon_{s},\left[\gamma_{\hat{x}}^{*}\right] \cap\left[\phi_{s}\right]\right)$. Assim, $\left[\gamma_{\hat{x}}^{*}\right] \subset \cup_{s \in[0,1]} V_{s}$, e por compacidade temos $\left[\gamma_{\hat{x}}^{*}\right] \subset \cup_{j=0}^{k} V_{s_{j}} \subset \cup_{j=0}^{k} B\left(\varepsilon_{s_{j}},\left[\gamma_{\hat{x}}^{*}\right] \cap\left[\phi_{s_{j}}\right]\right.$ ), para algum $k \in \mathbb{N}$ (note que o diâmetro de $B\left(\varepsilon_{s_{j}},\left[\gamma_{\hat{x}}^{*}\right] \cap\left[\phi_{s_{j}}\right]\right)$ é uniformemente limitado, mesmo para os valores de $s_{j}$ tais que $\left[\gamma_{\hat{x}}^{*}\right] \cap\left[\phi_{s_{j}}\right]$ é um segmento de folha, pois $\left.\sum d_{i} \leq 1\right)$. Assim, podemos particionar o intervalo $[0,1]$ em uma quantidade finita de subintervalos fechados e modificar $\gamma_{\hat{x}}^{*}$ em cada intervalo, dentro das vizinhanças tubulares, mantendo os extremos dos intervalos fixos, de forma a obtermos um caminho transverso $\gamma_{\hat{x}}$ tal que $\left[\gamma_{\hat{x}}\right] \subset \cup_{j=0}^{k} B\left(\varepsilon_{s_{j}},\left[\gamma_{\hat{x}}^{*}\right] \cap\left[\phi_{s_{j}}\right]\right)$ (veja a Figura 4.5) .

Note agora que como $\hat{\pi}(\hat{\beta})=\tilde{I}(\tilde{x})$ está contido em $U$ e, se $\phi$ é uma folha de $\tilde{\mathcal{F}}$, então 

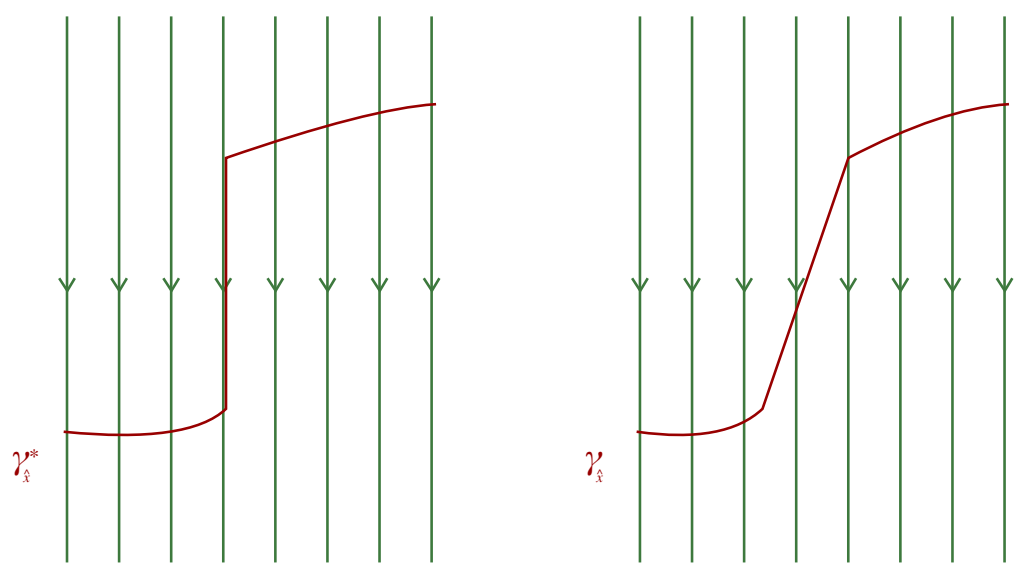

Figura 4.5: Modificação de $\gamma_{\hat{x}}^{*} e m \gamma_{\hat{x}}$

$[\phi] \cap U$ é conexo, então $\hat{\pi}\left(\gamma_{\hat{x}}^{*}\right)$ também está contido em $U$. Portanto, $\hat{\pi}\left(\cup_{j=0}^{k} B\left(\varepsilon_{s_{j}},\left[\gamma_{\hat{x}}^{*}\right] \cap\left[\phi_{s_{j}}\right]\right)\right) \subset$ $B\left(\max \left\{\varepsilon_{s_{j}}\right\}, U\right) \subset B(1, U)$, e portanto, denotanto $\hat{\pi}\left(\gamma_{\hat{x}}\right)=\tilde{\gamma}_{\tilde{x}}$, temos que $\tilde{\gamma}_{\tilde{x}} \subset B(1, U)$ e $\tilde{\gamma}_{\tilde{x}} \in \tilde{I}_{\tilde{\mathcal{F}}}(\tilde{x})$. Como $U$ é limitado, temos assim que existe $L_{0}>0$ tal que $\operatorname{diam}\left(\tilde{\gamma}_{\tilde{x}}\right)<L_{0}$, provando o resultado.

Denotaremos por $\tilde{\gamma}_{0}$ um representante de $\tilde{I}_{\tilde{\mathcal{F}}}^{\mathbb{Z}}\left(\tilde{z}_{0}\right)$ que passa por $\tilde{z}_{0}$ e $\left[\tilde{\gamma}_{0}\right]$ sua imagem. Usando o Lema 4.2 , podemos assumir que para cada $n \in \mathbb{Z}$, o segmento de $\tilde{\gamma}_{0}$ entre $\tilde{f}^{n}\left(\tilde{z}_{0}\right)$ e $\tilde{f}^{n+1}\left(\tilde{z}_{0}\right)$ tem diâmetro menor que $L_{0}$. Podemos assumir ainda que $\tilde{\gamma}_{0}$ está parametrizada de forma que $\tilde{\gamma}_{0}(n)=\tilde{f}^{n}\left(\tilde{z}_{0}\right)$, para todo $n \in \mathbb{Z}$.

Definição 4.3. Seja $v \in \mathbb{R}^{2}$ um vetor unitário tal que $v \neq \rho_{0}$ e $v \neq \rho_{0}^{\perp}$, e denotemos por $v_{s} 0$ vetor simétrico a $v$ com respeito à direção de $\rho_{0}$ (isto é, $\left\langle v_{s}, \rho_{0}\right\rangle=\left\langle v, \rho_{0}\right\rangle$ e $\left\langle v_{s}, \rho_{0}^{\perp}\right\rangle=-\left\langle v, \rho_{0}^{\perp}\right\rangle$ ). Denotemos agora as retas geradas por $v$ e $v_{s}$ que passam por $\tilde{z}_{0}$ por $r_{v}$ e $r_{v_{s}}$ (isto é, $r_{v}(t)=\tilde{z}_{0}+t v$, para $t \in \mathbb{R})$. Temos que $\mathbb{R}^{2} \backslash\left(\left[r_{v}\right] \cup\left[r_{v_{s}}\right]\right)$ possui quatro componentes conexas, e denotemos por $C_{1}$ e $C_{2}$ as componentes que intersectam a reta gerada por $\rho_{0}$ que passa por $\tilde{z}_{0}$. Chamaremos de cone gerado por $\rho_{0}$ com incliação $v$ e origem $\tilde{z}_{0}$ ao fecho de $C_{1} \cup C_{2}$, e denotaremos tal conjunto por $C_{\tilde{z}_{0}}^{\rho_{0}}(v)$ (veja Figura 4.6).

Note que na Definição 4.3 temos que $\partial\left(C_{\tilde{z}_{0}}^{\rho_{0}}(v)\right)=\left[r_{v}\right] \cup\left[r_{v_{s}}\right]$

No próximo lema denotaremos por $B\left(L_{0}, A\right)=\cup_{\tilde{x} \in A} B\left(L_{0}, \tilde{x}\right)$, onde $A \subset \mathbb{R}^{2}$.

Lema 4.4. Dado $v \in \mathbb{R}^{2}$ como na Definição 4.3, existe $L_{1}>0$ tal que $\left[\tilde{\gamma}_{0}\right] \subset B\left(L_{0}, C_{\tilde{z}_{0}}^{\rho_{0}}(v)\right) \cup$ $B\left(L_{1}, \tilde{z}_{0}\right)$, onde $L_{0}$ é dado pelo Lema 4.2.

Demonstração. Primeiramente, consideremos as retas $r_{v}$ e $r_{v_{s}}$, como na Definição 4.3. Denotando por $d\left(r_{v}, x\right)$ a distância entre a reta $r_{v}$ e o ponto $\tilde{x}$, temos que $d_{n}=d\left(r_{v}, n \rho_{0}+\tilde{z}_{0}\right)=d\left(r_{v_{s}}, n \rho_{0}+\tilde{z}_{0}\right)=$ $n\left\|\left\langle\rho_{0}, v\right\rangle v-\rho_{0}\right\|$, para $n \in \mathbb{Z}$. Note que $B\left(d_{n}, n \rho_{0}+\tilde{z}_{0}\right) \subset C_{\tilde{z}_{0}}^{\rho_{0}}(v)$.

Temos que $\lim _{n \rightarrow \infty} \frac{\tilde{f}^{n}\left(\tilde{z}_{0}\right)-\tilde{z}_{0}}{n}=\rho_{0}$. Assim, fazendo $\varepsilon=\left\|\left\langle\rho_{0}, v\right\rangle v-\rho_{0}\right\|$, existe $n_{1}>0$ tal que

$$
\begin{aligned}
d\left(\tilde{f}^{n}\left(\tilde{z}_{0}\right), n \rho_{0}+\tilde{z}_{0}\right) & =\left\|\tilde{f}^{n}\left(\tilde{z}_{0}\right)-\tilde{z}_{0}-n \rho_{0}\right\| \\
& <n \varepsilon=n\left\|\left\langle\rho_{0}, v\right\rangle v-\rho_{0}\right\|=d\left(r_{v}, n \rho_{0}+\tilde{z}_{0}\right), \quad \forall n \geq n_{1}
\end{aligned}
$$




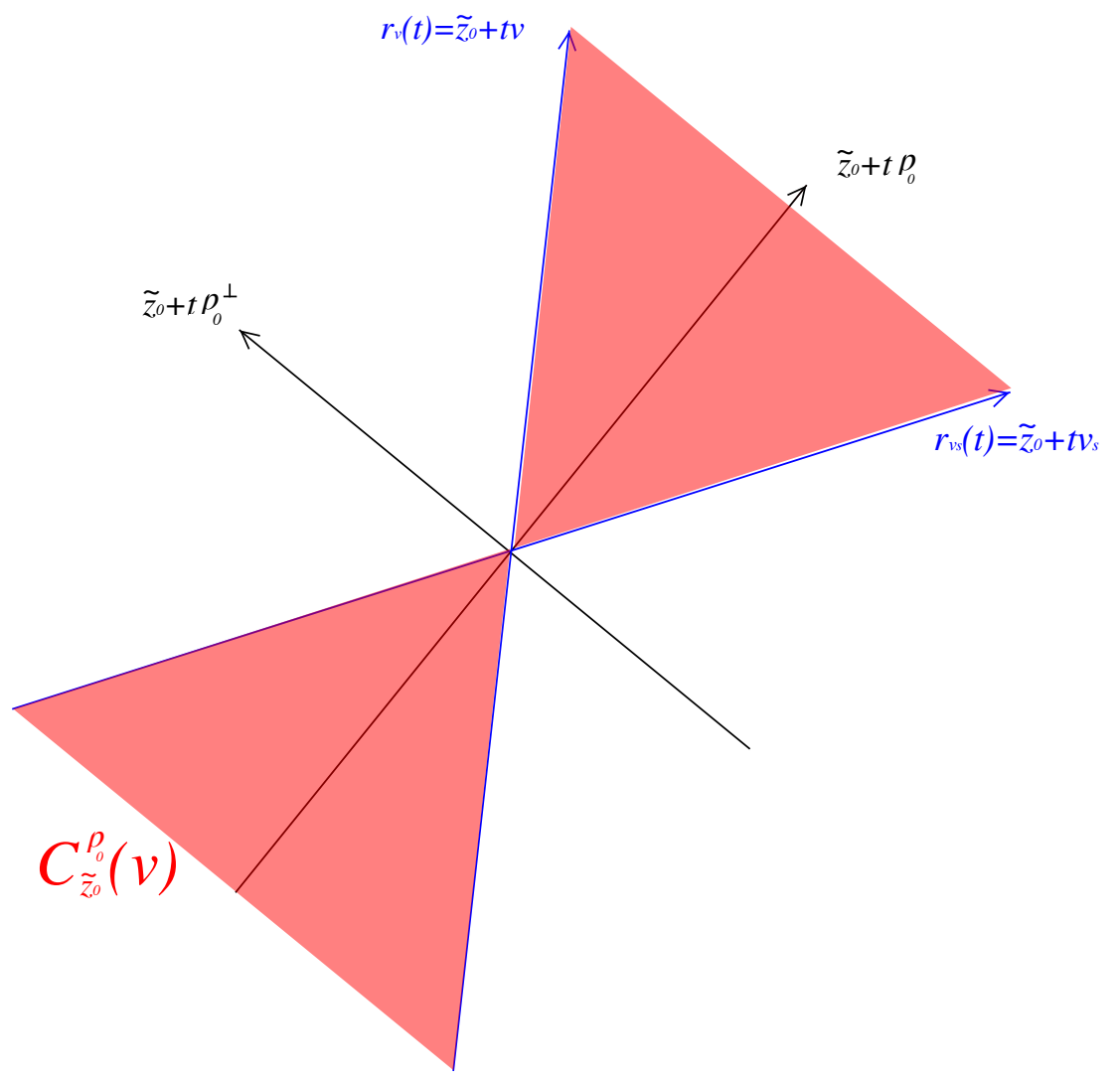

Figura 4.6: Ilustração do cone $C_{\tilde{z}_{0}}^{\rho_{0}}(v)$

Procedendo de forma análoga para $\tilde{f}^{-1}$, obtemos

$$
\begin{aligned}
d\left(\tilde{f}^{-n}\left(\tilde{z}_{0}\right),-n \rho_{0}+\tilde{z}_{0}\right) & =\left\|\tilde{f}^{-n}\left(\tilde{z}_{0}\right)-\tilde{z}_{0}-n\left(-\rho_{0}\right)\right\| \\
& <n \varepsilon=n\left\|\left\langle\rho_{0}, v\right\rangle v-\rho_{0}\right\|=d\left(r_{v}, n \rho_{0}+\tilde{z}_{0}\right), \quad \forall n \geq n_{2} .
\end{aligned}
$$

Assim, fazendo $n_{0}=\max \left\{n_{1}, n_{2}\right\}$, temos que $d\left(\tilde{f}^{n}\left(\tilde{z}_{0}\right), n \rho_{0}+\tilde{z}_{0}\right)<d_{n}=d\left(r_{v}, n \rho_{0}+\tilde{z}_{0}\right)$, e como $B\left(d_{n}, n \rho_{0}+\tilde{z}_{0}\right) \subset C_{\tilde{z}_{0}}^{\rho_{0}}(v)$, temos que $\tilde{f}^{n}\left(\tilde{z}_{0}\right) \in C_{\tilde{z}_{0}}^{\rho_{0}}(v)$, para $|n| \geq n_{0}$ (veja a Figura 4.7$)$.

Por construção, temos que o diâmetro do segmento de $\tilde{\gamma}_{0}$ entre $\tilde{f}^{n}\left(\tilde{z}_{0}\right)$ e $\tilde{f}^{n+1}\left(\tilde{z}_{0}\right)$ é menor que $L_{0}$, assim temos que para $|n| \geq n_{0}$ tal segmento está contido em $B\left(L_{0}, C_{\tilde{z}_{0}}^{\rho_{0}}(v)\right)$.

Para $n$ tal que $|n|<n_{0}$, temos que $\tilde{f}^{n}\left(\tilde{z}_{0}\right)$ pode estar fora do cone, mas como tais pontos existem apenas em quantidade finita, temos que existe $L^{\prime}>0$ tal que $\left\|\tilde{f}^{n}\left(\tilde{z}_{0}\right)-\tilde{z}_{0}\right\|<L^{\prime}$, para $|n|<n_{0}$. Novamente, como cada segmento de $\tilde{\gamma}_{0}$ tem diâmetro limitado por $L_{0}$, temos que os segmentos de $\tilde{\gamma}_{0}$ entre $\tilde{f}^{n}\left(\tilde{z}_{0}\right)$ e $\tilde{f}^{n+1}\left(\tilde{z}_{0}\right)$, com $|n|<n_{0}$, estão contidos em $B\left(L_{1}, \tilde{z}_{0}\right)$, onde $L_{1}=L^{\prime}+L_{0}$.

Lema 4.5. Se $\tilde{\alpha}:[a, b] \rightarrow \mathbb{R}^{2}$ é um caminho admissível para $\tilde{f}$, não existe $w \in \mathbb{Z}_{*}^{2}$ tal que $\tilde{\alpha} \pitchfork_{\tilde{\mathcal{F}}}$ $(\tilde{\alpha}+w)$.

Demonstração. Suponhamos que existem $w \in \mathbb{Z}_{*}^{2}$ e $\tilde{\alpha}:[a, b] \rightarrow \mathbb{R}^{2}$ um caminho $r$-admissível tais que $\alpha \pitchfork_{\tilde{\mathcal{F}}}(\tilde{\alpha}+w)$. Dessa forma, temos pelo Teorema 3.28 que dado $p / q \in(0,1]$ escrito em forma irredutível, $f$ possuirá ponto periódico com vetor de rotação igual a $\frac{p}{q r} \cdot w$, o que é um absurdo pois $\rho(\tilde{f}) \cap \mathbb{Q}^{2}=\{(0,0)\}$.

Lema 4.6. Não existe $w \in \mathbb{Z}^{2}$ tal que $\tilde{\gamma}_{0} \pitchfork_{\tilde{\mathcal{F}}}\left(\tilde{\gamma}_{0}+w\right)$.

Demonstração. Se $w \neq 0$, o resultado é consequência direta do Lema 4.5. Para $w=0$, suponhamos por absurdo que $\tilde{\gamma}_{0}$ possua uma autointersecção transversa. Assim, temos que existem intervalos 


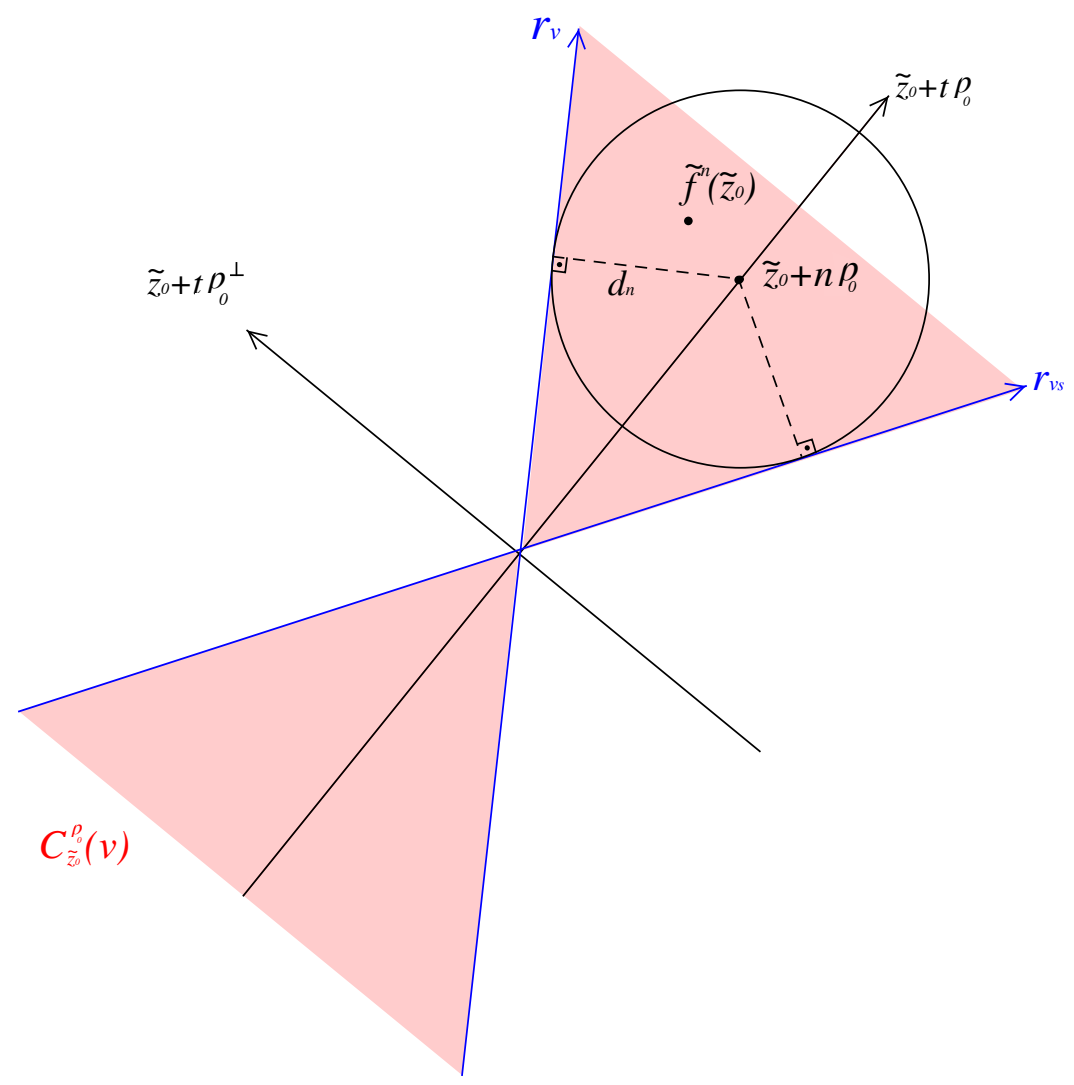

Figura 4.7: Ilustração de $\tilde{f}^{n}\left(\tilde{z}_{0}\right) \in C_{\tilde{z}_{0}}^{\rho_{0}}(v)$

$I, J \subset \mathbb{R}$ tais que $\left.\left.\tilde{\gamma}_{0}\right|_{I} \pitchfork_{\tilde{\mathcal{F}}} \tilde{\gamma}_{0}\right|_{J}$. Podemos supor que $I \subset(-N, N)$, para algum $N \in \mathbb{N}$. Agora notemos que, como $z_{0}$ é recorrente, existem $n_{k} \in \mathbb{N}$ e $w_{k} \in \mathbb{Z}^{2}$ tais que $\tilde{f}^{n_{k}}\left(\tilde{z}_{0}\right) \rightarrow \tilde{z}_{0}+w_{k}$ ou, equivalentemente, $\tilde{f}^{n_{k}}\left(\tilde{z}_{0}\right)-w_{k} \rightarrow \tilde{z}_{0}$, e portanto $\tilde{f}^{-N+n_{k}}\left(\tilde{z}_{0}\right)-w_{k} \rightarrow \tilde{f}^{-N}\left(\tilde{z}_{0}\right)$. Além disso, como $\rho\left(f, z_{0}\right)=\rho_{0}$, temos que $w_{k} / n_{k} \rightarrow \rho_{0}$ e portanto existe $k_{0}$ tal que $w_{k} \neq 0$ se $k>k_{0}$.

Vejamos agora que, pela forma como $\tilde{\gamma}_{0}$ foi parametrizada, temos que $\tilde{\gamma}_{0}(-N)=\tilde{f}^{-N}\left(\tilde{z}_{0}\right)$ e $\tilde{\gamma}_{0}(N)=\tilde{f}^{N}\left(\tilde{z}_{0}\right)$, e portanto $\left.\tilde{\gamma}_{0}\right|_{[-N, N]}=\tilde{I}_{\tilde{\mathcal{F}}}^{2 N}\left(\tilde{f}^{-N}\left(\tilde{z}_{0}\right)\right)$. Como $\tilde{f}^{-N+n_{k}}\left(\tilde{z}_{0}\right)-w_{k} \rightarrow \tilde{f}^{-N}\left(\tilde{z}_{0}\right)$, pelo Lema 3.23 temos que se $k^{\prime}$ for suficientemente grande, $\tilde{I}_{\tilde{\mathcal{F}}}^{2 N}\left(\tilde{f}^{-N}\left(\tilde{z}_{0}\right)\right)$ é equivalente a um subcaminho de $\tilde{I}_{\tilde{\mathcal{F}}}^{2 N+2}\left(\tilde{f}^{-1}\left(\tilde{f}^{-N+n_{k^{\prime}}}\left(\tilde{z}_{0}\right)-w_{k^{\prime}}\right)\right)$. Mas

$$
\begin{aligned}
\tilde{I}_{\tilde{\mathcal{F}}}^{2 N+2}\left(\tilde{f}^{-1}\left(\tilde{f}^{-N+n_{k^{\prime}}}\left(\tilde{z}_{0}\right)-w_{k^{\prime}}\right)\right) & =\tilde{I}_{\tilde{\mathcal{F}}}^{2 N+2}\left(\tilde{f}^{-N-1+n_{k^{\prime}}}\left(\tilde{z}_{0}\right)-w_{k^{\prime}}\right) \\
& =\left.\left(\tilde{\gamma}_{0}-w_{k^{\prime}}\right)\right|_{\left[-N-1+n_{k^{\prime}}, N+1+n_{k^{\prime}}\right]}
\end{aligned}
$$

e podemos supor também que $k^{\prime}>k_{0}$. Assim, existe $I^{\prime} \subset\left[-N-1+n_{k^{\prime}}, N+1+n_{k^{\prime}}\right]$ tal que $\left.\left.\left(\tilde{\gamma}_{0}-w_{k^{\prime}}\right)\right|_{I^{\prime}} \sim_{\tilde{\mathcal{F}}} \tilde{\gamma}_{0}\right|_{[-N, N]}$. Como $I \subset[-N, N]$, temos que existe $I^{\prime \prime} \subset I^{\prime}$ tal que $\left.\left.\left(\tilde{\gamma}_{0}-w_{k^{\prime}}\right)\right|_{I^{\prime \prime}} \sim_{\tilde{\mathcal{F}}} \tilde{\gamma}_{0}\right|_{I}$, mas $\left.\left.\tilde{\gamma}_{0}\right|_{I} \pitchfork_{\tilde{\mathcal{F}}} \tilde{\gamma}_{0}\right|_{J}$, portanto $\left.\left.\left(\tilde{\gamma}_{0}-w_{k^{\prime}}\right)\right|_{I^{\prime}} \pitchfork_{\tilde{\mathcal{F}}} \tilde{\gamma}_{0}\right|_{J}$, e dado que $k^{\prime}>k_{0}$, temos $w_{k^{\prime}} \neq 0$, e assim obtemos um absurdo, pelo Lema 4.5.

Lema 4.7. $\tilde{\gamma}_{0}$ intersecta cada folha no máximo uma vez.

Demonstração. Suponhamos, por absurdo, que existem $t^{\prime}<t^{\prime \prime}$ tais que $\phi_{\tilde{\gamma}_{0}\left(t^{\prime}\right)}=\phi_{\tilde{\gamma}_{0}\left(t^{\prime \prime}\right)}$. Portanto temos que $\left.\tilde{\gamma}_{0}\right|_{\left[t^{\prime}, t^{\prime \prime}\right]}$ é $\tilde{\mathcal{F}}$-equivalente a uma curva transversa e fechada $\Gamma$. Mas como $\Gamma$ possui algum subcaminho $\Gamma_{0}: J \rightarrow \mathbb{R}^{2}$ que é uma curva transversa, fechada e simples, temos que $\tilde{\gamma}_{0}$ possui um subcaminho $\tilde{\mathcal{F}}$-equivalente a $\Gamma_{0}$. Como $\tilde{\gamma}_{0}$ não possui autointersecção transversa, segue da Proposição 20 de [CT18b] temos:

(i) $\bigcup_{s \in J}\left[\phi_{\Gamma_{0}(s)}\right]=A_{\Gamma_{0}}$ é um anel topológico aberto; 
(ii) $\left\{t \in \mathbb{R} \mid \tilde{\gamma}_{0}(t) \in A_{\Gamma_{0}}\right\}$ é um intervalo, que denotaremos por $I=(a, b)$, não necessariamente limitado;

(iii) se $-\infty<a<b<+\infty$, então $\tilde{\gamma}_{0}\left(t^{\prime}\right)$ e $\tilde{\gamma}_{0}\left(t^{\prime \prime}\right)$ não podem ambas pertencerem a componentes conexas ilimitadas $\mathbb{R}^{2} \backslash A_{\Gamma_{0}}$.

Como $z_{0}$ é recorrente e tem vetor de rotação $\rho$ temos, como na prova do Lema 4.6, que existem $t_{k} \rightarrow+\infty, w_{k}^{+} \in \mathbb{Z}^{2}$, com $\left\|w_{k}^{+}\right\| \rightarrow+\infty$ e $s_{k} \rightarrow+\infty, v_{k}^{-} \in \mathbb{Z}^{2}$, com $\left\|v_{k}^{-}\right\| \rightarrow+\infty$ tais que $\tilde{\gamma}_{0}\left(t_{k}\right) \in A_{\Gamma_{0}}+w_{k}^{+}$e $\tilde{\gamma}_{0}\left(s_{k}\right) \in A_{\Gamma_{0}}+v_{k}^{-}$. Primeiramente notemos que, como $\Gamma$ é uma curva transversa, fechada e simples, toda folha $\phi$ que intersecta $\Gamma_{0}$ o faz em apenas um ponto. Disto segue que ou toda folha que intersecta $\Gamma$ tem seu $\omega$-limite contido na componente conexa limitada do seu complementar, ou então toda folha que intersecta $\Gamma_{0}$ tem seu $\alpha$-limite contido na componente conexa limitada do seu complementar. Iremos assumir, sem perda de generalidade, que a primeira situação ocorre, sendo que o segundo caso é tratado de forma análoga. Mas pela invariância da folheação por translações inteiras segue que uma propriedade análoga vale para as folhas que intersectam $\Gamma_{0}+w$, para vetores inteiros $w$. Temos portanto que, se $[\phi] \subset A_{\Gamma_{0}}$ e $\left[\Gamma_{0}+w\right] \cap\left[\Gamma_{0}\right]=\emptyset$, então temos que $[\phi] \cap A_{\Gamma_{0}}+w=\emptyset$, pois o $\omega$-limite de uma folha na intersecção estaria contido em dois conjuntos disjuntos.

Assim, para $k$ grande o suficiente, como $\left[\Gamma_{0}+w_{k}^{+}\right] \cap\left[\Gamma_{0}\right]=\emptyset$, temos que $\phi_{\tilde{\gamma}_{0}\left(t_{k}\right)}$ está contida numa componente conexa ilimitada de $\mathbb{R}^{2} \backslash A_{\Gamma_{0}}$, e o mesmo vale para $\phi_{\tilde{\gamma}_{0}\left(s_{k}\right)}$. Assim, pela Proposição 20 de [CT18b], temos que $\tilde{\gamma}_{0}$ possui autointersecção transversa, o que é um absurdo.

Corolário 4.8. $\tilde{\gamma}_{0}$ é uma linha.

Demonstração. Pelo Lema 4.7 temos que $\tilde{\gamma}_{0}$ é um caminho simples, e como $\left\|\tilde{f}^{n}\left(\tilde{z}_{0}\right)\right\|$ converge para infinito quando $n \rightarrow \pm \infty$ temos o resultado.

Portanto, como $\tilde{\gamma}_{0}$ é uma linha, estão bem definidos os conjuntos $L\left(\tilde{\gamma}_{0}\right), R\left(\tilde{\gamma}_{0}\right), l\left(\tilde{\gamma}_{0}\right)$ e $r\left(\tilde{\gamma}_{0}\right)$.

Lema 4.9. Seja A uma componente conexa de $r\left(\tilde{\gamma}_{0}\right)$ (ou $l\left(\tilde{\gamma}_{0}\right)$ ). Então A é um conjunto ilimitado.

Demonstração. Pelo Lema 4.7 , temos que $\tilde{\gamma}_{0}$ é uma linha e cruza cada folha no máximo uma única vez. Assim, o conjunto $\bigcup_{t \in \mathbb{R}}\left[\phi_{\tilde{\gamma}_{0}(t)}\right]$ das folhas que passam por $\tilde{\gamma}_{0}$ é homeomorfo a $\mathbb{R}^{2}$ e portanto simplesmente conexo. Logo, todas as componentes conexas do complementar de tal conjunto são ilimitadas.

Lema 4.10. Se $K \subset \mathbb{R}^{2}$ é compacto, então o conjunto $I_{K}=\left\{t \in \mathbb{R} \mid\left[\phi_{\tilde{\gamma}_{0}(t)}\right] \cap K \neq \emptyset\right\}$ também é compacto

Demonstração. Sejam $\phi$ uma folha tal que $\phi(\bar{s}) \in K$, para algum $\bar{s} \in \mathbb{R}$, e $V_{-}^{w}, V_{+}^{w} \in \mathbb{Z}^{2}$ tais que $K \subset R\left(\alpha_{-}+V_{-}^{w}\right)$ e $K \subset L\left(\alpha_{+}+V_{+}^{w}\right)$ (veja a Figura 4.8), sendo $\alpha_{-}$e $\alpha_{+}$as linhas transversas dadas pelo Lema 4.1 (note que podemos encontrar tais linhas pois $K$ é compacto, e portanto limitado). Temos então que $\phi(\bar{s}) \in R\left(\alpha_{-}+V_{-}^{w}\right) \cap L\left(\alpha_{+}+V_{+}^{w}\right)$, e portanto, pela Observação 3.12, $\phi(s) \in R\left(\alpha_{-}+V_{-}^{w}\right)$, para todo $s>\bar{s}$, e $\phi(s) \in L\left(\alpha_{+}+V_{+}^{w}\right)$, para todo $s<\bar{s}$, isto é, $[\phi] \subset$ $R\left(\alpha_{-}+V_{-}^{w}\right) \cup L\left(\alpha_{+}+V_{+}^{w}\right)$. Contudo, como $\alpha_{-}$tem vetor diretor $v_{-}$tal que $\left\langle v_{-}, \rho_{0}^{\perp}\right\rangle<0$ e $\left\langle v_{-}, \rho_{0}\right\rangle>0$ (e $\alpha_{+}$tem vetor diretor $v_{+}$tal que $\left\langle v_{+}, \rho_{0}^{\perp}\right\rangle>0$ e $\left\langle v_{+}, \rho_{0}\right\rangle>0$ ) e, pelo Lema 4.4, $\left[\tilde{\gamma}_{0}+w\right]$ está contido em $B\left(L_{0}, C_{\tilde{z}_{0}}^{\rho_{0}}(v)+w\right) \cup B\left(L_{1}, \tilde{z}_{0}+w\right)$, onde o cone $C_{\tilde{z}_{0}}^{\rho_{0}}(v)$ é gerado por $\rho_{0}$ e $v$ é um vetor unitário tal que $\left\langle v, \rho_{0}\right\rangle>0$ e $0<\left\langle v, \rho_{0}^{\perp}\right\rangle\left\langle\left\langle v_{+}, \rho_{0}^{\perp}\right\rangle\right.$, temos que existe $\bar{t}=\max \{t \in \mathbb{R} \mid$ $\left.\left(\tilde{\gamma}_{0}+w\right)(t) \in R\left(\alpha_{-}+V_{-}^{w}\right) \cup L\left(\alpha_{+}+V_{+}^{w}\right)\right\}$, e assim $\phi$ só pode cruzar $\tilde{\gamma}_{0}+w$ antes de $\bar{t}$. Procedendo de forma simétrica podemos obter um limitante inferior para o instante em que $\phi$ pode cruzar $\tilde{\gamma}_{0}+w$, provando assim a afirmação.

Corolário 4.11. Sejam $\zeta:[a, b] \rightarrow \mathbb{R}^{2}$ um caminho transverso e $w \in \mathbb{Z}^{2}$. Se $[\zeta] \cap l\left(\tilde{\gamma}_{0}+w\right) \neq \emptyset$ e $[\zeta] \cap r\left(\tilde{\gamma}_{0}+w\right) \neq \emptyset$, então $\zeta \pitchfork_{\tilde{\mathcal{F}}}\left(\tilde{\gamma}_{0}+w\right)$. 


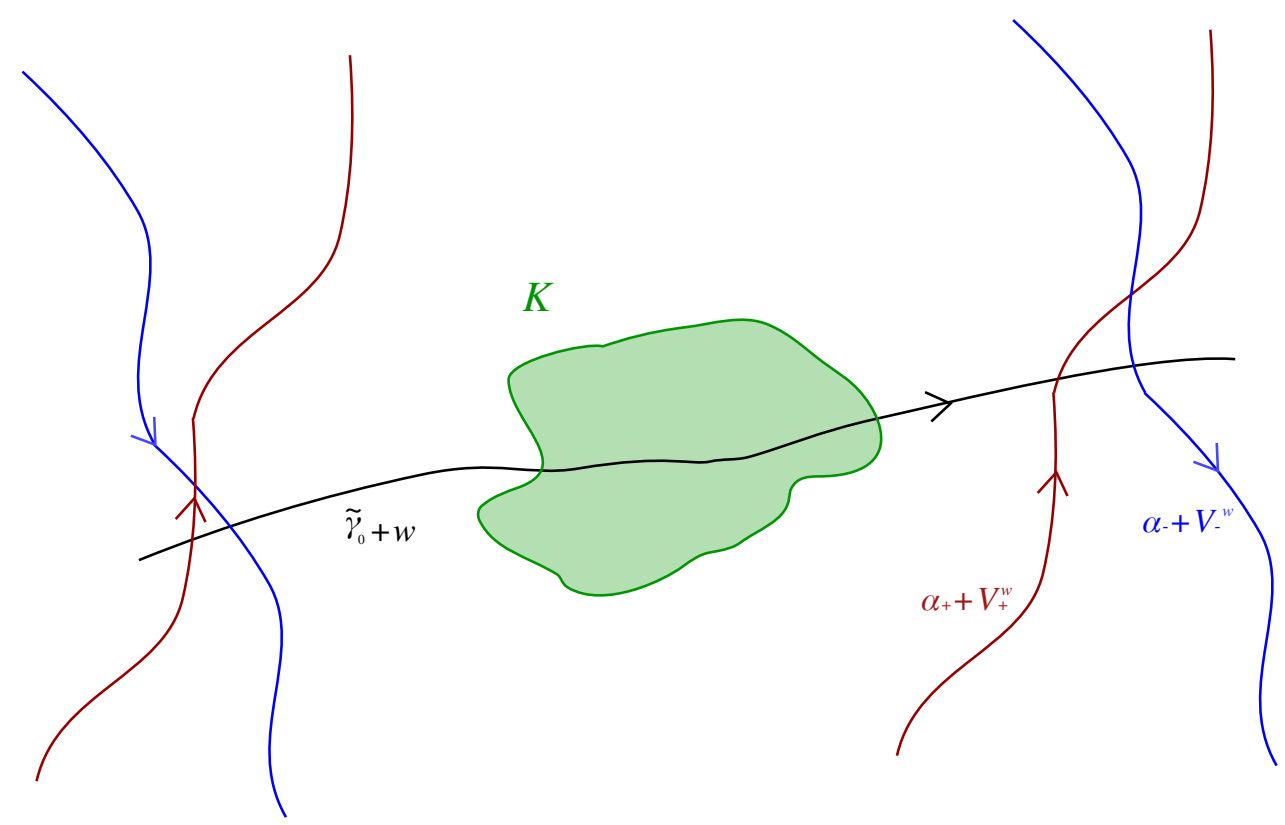

Figura 4.8: Demonstração do Lema 4.10

Demonstração. Pela Proposição 3.22, basta provar que o conjunto $I_{\zeta}=\left\{t \in \mathbb{R} \mid \phi_{\left(\tilde{\gamma}_{0}+w\right)(t)}\right.$ cruza $\left.\zeta\right\}$ é compacto. Mas isso segue diretamente do Lema 4.10.

Lema 4.12. Se $\tilde{\gamma}^{\prime}: \mathbb{R} \rightarrow \mathbb{R}^{2}$ é tal que $\tilde{\gamma}^{\prime} \sim_{\tilde{\mathcal{F}}} \tilde{\gamma}_{0}$, então $\tilde{\gamma}^{\prime}$ é uma linha.

Demonstração. Pelo Lema 4.7, como $\tilde{\gamma}^{\prime} \sim_{\tilde{\mathcal{F}}} \tilde{\gamma}_{0}$, temos que $\tilde{\gamma}^{\prime}$ intersecta cada folha no máximo uma vez, portanto $\tilde{\gamma}^{\prime}$ é uma curva simples. Agora seja $K \subset \mathbb{R}^{2}$ um conjunto compacto. Como $\tilde{\gamma}^{\prime} \sim_{\tilde{\mathcal{F}}} \tilde{\gamma}_{0}$, temos que existe uma reparametrização de $\tilde{\gamma}^{\prime}$ de forma que $\phi_{\tilde{\gamma}_{0}(t)}=\phi_{\tilde{\gamma}^{\prime}(t)}$, para todo $t \in \mathbb{R}$. Note ainda que $\tilde{\gamma}^{\prime}$ ser própria é uma propriedade que não depende da parametrização da mesma. Assim, pelo Lema 4.10, temos que existe $M>0$ de forma que $\phi_{\tilde{\gamma}^{\prime}(t)} \cap K=\emptyset$, para todo $|t|>M$. Assim, se $t^{\prime} \in\left(\tilde{\gamma}^{\prime}\right)^{-1}(K)$, temos que $\left|t^{\prime}\right|<M$, e assim temos que $\tilde{\gamma}^{\prime}$ é uma curva própria, provando o lema.

Lema 4.13. Dado $w \in \mathbb{Z}_{*}^{2}$, se $\tilde{\gamma}_{0}$ e $\tilde{\gamma}_{0}+w$ intersectam uma mesma folha $\phi$, então existem sequências $t_{k}^{+}, s_{k}^{+} \nearrow+\infty, t_{k}^{-}, s_{k}^{-} \searrow-\infty$ e curvas transversas $\tilde{\gamma}_{i}^{\prime}: \mathbb{R} \rightarrow \mathbb{R}^{2}, i=1,2$ tais que:

(i) $\tilde{\gamma}_{1}^{\prime} \sim_{\tilde{\mathcal{F}}} \tilde{\gamma}_{0}$ e $\tilde{\gamma}_{2}^{\prime} \sim_{\tilde{\mathcal{F}}} \tilde{\gamma}_{0}+w$;

(ii) $\tilde{\gamma}_{1}^{\prime}\left(t_{k}^{+}\right)=\tilde{\gamma}_{2}^{\prime}\left(s_{k}^{+}\right)$e $\tilde{\gamma}_{1}^{\prime}\left(t_{k}^{-}\right)=\tilde{\gamma}_{2}^{\prime}\left(s_{k}^{-}\right)$, para todo $k \in \mathbb{N}$.

Demonstração. Como $\tilde{\gamma}_{0}$ é recorrente, podemos encontrar sequências $t_{k}^{+}, s_{k}^{+} \nearrow+\infty$, tais que $\tilde{\gamma}_{0}\left(t_{k}^{+}\right)$ e $\left(\tilde{\gamma}_{0}+w\right)\left(s_{k}^{+}\right)$estão na folha $\phi_{k}^{+}=\phi+w_{k}^{+}$, para algum $w_{k}^{+} \in \mathbb{Z}^{2}$ (e da mesma forma, sequências $t_{k}^{-}, s_{k}^{-} \searrow-\infty$ com a mesma propriedade). Sejam, para cada $k \in \mathbb{N}, W_{k}^{+}$uma vizinhança tubular da folha $\phi+w_{k}^{+}$de forma que $\tilde{\gamma}_{0}\left(t_{k}^{+}\right),\left(\tilde{\gamma}_{0}+w\right)\left(s_{k}^{+}\right) \in W_{k}^{+}$e $W_{k}^{-}$uma vizinhança tubular da folha $\phi+w_{k}^{-}$de forma que $\tilde{\gamma}_{0}\left(t_{k}^{-}\right),\left(\tilde{\gamma}_{0}+w\right)\left(s_{k}^{-}\right) \in W_{k}^{-}$. Como $\left\|w_{k}^{ \pm}\right\| \rightarrow \infty$, podemos assumir que as vizinhanças são mutuamente disjuntas. Assim, fazendo em cada vizinhança uma modificação nos caminhos similar à feita no Lema 4.1 (veja a Figura 4.2) podemos obter caminhos transversos $\tilde{\gamma}_{1}^{\prime} \mathrm{e}$ $\tilde{\gamma}_{2}^{\prime}$ que satisfazem as propriedades $(i)$ e $(i i)$ do enunciado.

Observação 4.14. Note que pelo Lema 4.12, temos que $\tilde{\gamma}_{1}^{\prime}$ e $\tilde{\gamma}_{2}^{\prime}$ são linhas.

Lema 4.15. Se $\tilde{\gamma}_{0}$ e $\tilde{\gamma}_{0}+w$ cruzam uma mesma folha, então toda componente conexa de $L\left(\tilde{\gamma}_{1}^{\prime}\right) \cap$ $R\left(\tilde{\gamma}_{2}^{\prime}\right)$ e de $R\left(\tilde{\gamma}_{1}^{\prime}\right) \cap L\left(\tilde{\gamma}_{2}^{\prime}\right)$ é limitada, onde $\tilde{\gamma}_{1}^{\prime}$ e $\tilde{\gamma}_{2}^{\prime}$ são as curvas dadas pelo Lema 4.13. 
Demonstração. Assumamos, por contradição, que o resultado não é verdadeiro. Podemos, sem perda de generalidade, assumir que existe uma componente conexa ilimitada $O$ de $R\left(\tilde{\gamma}_{1}^{\prime}\right) \cap L\left(\tilde{\gamma}_{2}^{\prime}\right)$. O caso onde existe uma componente conexa ilimitada de $L\left(\tilde{\gamma}_{1}^{\prime}\right) \cap R\left(\tilde{\gamma}_{2}^{\prime}\right)$ é similar. Como $\tilde{\gamma}_{1}^{\prime}$ e $\tilde{\gamma}_{2}^{\prime}$ são linhas e se intersectam, toda componente conexa do complementar de $\left[\tilde{\gamma}_{1}^{\prime}\right] \cup\left[\tilde{\gamma}_{2}^{\prime}\right]$ possui em sua fronteira pontos que estão em $\left[\tilde{\gamma}_{1}^{\prime}\right]$ mas não em $\left[\tilde{\gamma}_{2}^{\prime}\right]$ e também pontos que estão em $\left[\tilde{\gamma}_{2}^{\prime}\right]$ mas não em [ $\left.\tilde{\gamma}_{1}^{\prime}\right]$. Sejam $P_{1}$ e $P_{2}$ pontos em $\partial O \cap\left[\tilde{\gamma}_{1}^{\prime}\right] \cap\left(\mathbb{R}^{2} \backslash\left[\tilde{\gamma}_{2}^{\prime}\right]\right)$ e $\partial O \cap\left(\mathbb{R}^{2} \backslash\left[\tilde{\gamma}_{1}^{\prime}\right]\right) \cap\left[\tilde{\gamma}_{2}^{\prime}\right]$, respectivamente. Sejam $\phi_{1}, \phi_{2}: \mathbb{R} \rightarrow \operatorname{dom}(\tilde{I})$ as folhas de $\tilde{\mathcal{F}}$ que passam por $P_{1}$ e $P_{2}$ respectivamente, e assumamos que $\phi_{1}(0)=P_{1}$ e $\phi_{2}(0)=P_{2}$. Note que $\phi_{1}(0) \in L\left(\tilde{\gamma}_{2}^{\prime}\right)$, e como toda folha de $\tilde{\mathcal{F}}$ que intersecta $\tilde{\gamma}_{1}^{\prime}$ ou $\tilde{\gamma}_{2}^{\prime}$ deve cruzar tal caminho da esquerda para a direita, temos que $\phi_{1}((-\infty, 0))$ está contido em $L\left(\tilde{\gamma}_{1}^{\prime}\right) \cup L\left(\tilde{\gamma}_{2}^{\prime}\right)$. Além disso, se $\phi_{1}((-\infty, 0))$ é ilimitado, então o $\alpha$-limite de $\phi_{1}$ estará contido em $l\left(\tilde{\gamma}_{1}^{\prime}\right) \cup l\left(\tilde{\gamma}_{2}^{\prime}\right)$. Ainda, se $\varepsilon$ for suficientemente pequeno, então $\phi_{1}(\varepsilon)$ pertence a $O$. Analogamente, é possível mostrar que $\phi_{2}((0,+\infty))$ está contido em $R\left(\tilde{\gamma}_{1}^{\prime}\right) \cup R\left(\tilde{\gamma}_{2}^{\prime}\right)$, seu $\omega$-limite está contido em $r\left(\gamma_{1}\right) \cup r\left(\gamma_{2}\right)$ e, se $\varepsilon$ for suficientemente pequeno, então $\phi_{2}(-\varepsilon)$ pertence a $O$.

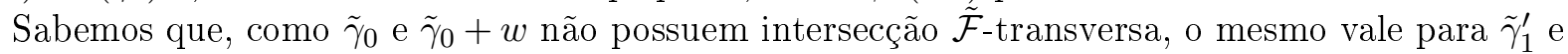
$\tilde{\gamma}_{2}^{\prime}$, já que estes caminhos são equivalentes a $\tilde{\gamma}_{0}$ e $\tilde{\gamma}_{0}+w$, respectivamente. Portanto, pelo Corolário 4.11, temos que $\tilde{\gamma}_{1}^{\prime}$ não pode intersectar tanto $r\left(\tilde{\gamma}_{2}^{\prime}\right)$ quanto $l\left(\tilde{\gamma}_{2}^{\prime}\right)$. Vamos assumir inicialmente que $\tilde{\gamma}_{1}^{\prime}$ não intersecta $r\left(\tilde{\gamma}_{2}^{\prime}\right)$.

Seja $t_{0}$ tal que $\tilde{\gamma}_{1}^{\prime}\left(t_{0}\right)=P_{1}$. Pelo Lema 4.13 existem $t_{-}<t_{0}<t_{+}$e $s_{-}<s_{+}$tais que $\tilde{\gamma}_{1}^{\prime}\left(t_{-}\right)=$ $\tilde{\gamma}_{2}^{\prime}\left(s_{-}\right)$e $\tilde{\gamma}_{1}^{\prime}\left(t_{+}\right)=\tilde{\gamma}_{2}^{\prime}\left(s_{+}\right)$. Seja $s_{-}^{\prime}$ o maior real menor que $s^{+}$tal que $\tilde{\gamma}_{2}^{\prime}\left(s_{-}^{\prime}\right)=\tilde{\gamma}_{1}^{\prime}\left(t_{-}^{\prime}\right)$, com $t_{-}^{\prime}<t_{0}$, e seja $s_{+}^{\prime}$ o menor real maior que $s^{\prime-}$ tal que $\tilde{\gamma}_{2}^{\prime}\left(s_{+}^{\prime}\right)=\tilde{\gamma}_{1}^{\prime}\left(t_{+}^{\prime}\right)$, com $t_{+}^{\prime}>t_{0}$. Temos que, orientados de forma adequada, $\tilde{\gamma}_{1}^{\prime}\left(\left[t_{-}^{\prime}, t_{+}^{\prime}\right]\right) \cup \tilde{\gamma}_{2}^{\prime}\left(\left[s_{-}^{\prime}, s_{+}^{\prime}\right]\right)$ é a imagem de uma curva fechada simples $C_{1}$, separando o plano em duas componentes conexas, sendo uma delas limitada, $P_{1}$ pertence a imagem desta curva, e se $\varepsilon$ é pequeno o suficiente então $\phi_{1}(-\varepsilon)$ e $\phi_{1}(\varepsilon)$ pertencem a componentes conexas distintas do complementar da curva. Mas temos que, se $H_{1}$ é a componente conexa de $l\left(\tilde{\gamma}_{2}^{\prime}\right)$ que contém o $\alpha$-limite de $\phi_{1}$, então $F_{1}=H_{1} \cup \phi_{1}((-\infty,-\varepsilon))$ não intersecta $\left[\tilde{\gamma}_{1}^{\prime}\right] \cup\left[\tilde{\gamma}_{2}^{\prime}\right]$. Além disso, pelo Lema 4.9 temos que $H_{1}$ é um conjunto ilimitado, portanto $F_{1}$ também é ilimitado, e assim está contido numa componente conexa ilimtada do complementar de $C_{1}$. Note ainda que $\phi_{1}(-\varepsilon)$ está na mesma componente conexa do complementar de $C_{1}$ que $F_{1}$. Contudo, $O$ está contido no complementar de $\left[\tilde{\gamma}_{1}^{\prime}\right] \cup\left[\tilde{\gamma}_{2}^{\prime}\right]$, e portanto no complementar de $C_{1}$, e $O$ também é ilimitado. Note que $\phi_{1}(\varepsilon) \in O$ e que $\phi_{1}(-\varepsilon)$ e $\phi_{1}(\varepsilon)$ estão em componentes conexas distintas do complementar de $C_{1}$. Portanto $O$ e $F_{1}$ estão contidos em componentes conexas distintas do complementar de $C_{1}$ e ambas são ilimitadas, o que é um absurdo.

Assumamos agora que $\tilde{\gamma}_{1}^{\prime}$ não intersecta $l\left(\tilde{\gamma}_{2}^{\prime}\right)$. Seja $s_{0}$ tal que $\tilde{\gamma}_{2}^{\prime}\left(s_{0}\right)=P_{2}$. Podemos, como antes, encontrar $t_{-}^{\prime \prime}<t_{+}^{\prime \prime}$ e $s_{-}^{\prime \prime}<s_{0}<s_{+}^{\prime \prime}$ tais que $\tilde{\gamma}_{1}^{\prime}\left(\left[t_{-}^{\prime \prime}, t_{+}^{\prime \prime}\right]\right) \cup \tilde{\gamma}_{2}^{\prime}\left(\left[s_{-}^{\prime \prime}, s_{+}^{\prime \prime}\right]\right)$ é a imagem de uma curva fechada simples $C_{2}$, e tais que se $\varepsilon$ é suficientemente pequeno, $\phi_{2}(-\varepsilon)$ e $\phi_{2}(\varepsilon)$ pertencem a componentes conexas distintas do complementar da curva $C_{2}$. Note que, se $H_{2}$ é a componente conexa de $r\left(\tilde{\gamma}_{2}^{\prime}\right)$ que contém o $\omega$-limite de $\phi_{2}$, então $F_{2}=H_{2} \cup \phi_{2}\left((\varepsilon,+\infty)\right.$ não intersecta $\left[\tilde{\gamma}_{1}^{\prime}\right] \cup\left[\tilde{\gamma}_{2}^{\prime}\right]$. Procedendo como anteriormente mostramos que as duas componentes conexas do complementar de $C_{2}$ são ilimitadas, obtendo assim uma contradição.

Lema 4.16. $\operatorname{Se} r\left(\tilde{\gamma}_{0}\right) \cap l\left(\tilde{\gamma}_{0}+w\right) \neq \emptyset$, então:

(i) $\left[\tilde{\gamma}_{0}\right] \cap l\left(\tilde{\gamma}_{0}+w\right) \neq \emptyset$;

(ii) $\left[\tilde{\gamma}_{0}+w\right] \cap r\left(\tilde{\gamma}_{0}\right) \neq \emptyset$.

Demonstração. Note que se $\tilde{\gamma}_{0}$ e $\tilde{\gamma}_{0}+w$ não cruzam uma mesma folha o resultado é trivial. Suponhamos então que $\tilde{\gamma}_{0}$ e $\tilde{\gamma}_{0}+w$ cruzam uma mesma folha, e sejam as linhas $\tilde{\gamma}_{1}^{\prime}$ e $\tilde{\gamma}_{2}^{\prime}$ dadas pelo Lema 4.13. Note que, como $\tilde{\gamma}_{1}^{\prime} \sim_{\tilde{\mathcal{F}}} \tilde{\gamma}_{0}$ e $\tilde{\gamma}_{2}^{\prime} \sim_{\tilde{\mathcal{F}}} \tilde{\gamma}_{0}+w$, temos $r\left(\tilde{\gamma}_{0}\right)=r\left(\tilde{\gamma}_{1}^{\prime}\right)$ e $l\left(\tilde{\gamma}_{0}+w\right)=l\left(\tilde{\gamma}_{2}^{\prime}\right)$. Seja $\tilde{p}^{\prime} \in r\left(\tilde{\gamma}_{1}^{\prime}\right) \cap l\left(\tilde{\gamma}_{2}^{\prime}\right)$. Como $r\left(\tilde{\gamma}_{1}^{\prime}\right) \subset R\left(\tilde{\gamma}_{1}^{\prime}\right)$ e $l\left(\tilde{\gamma}_{2}^{\prime}\right) \subset L\left(\tilde{\gamma}_{2}^{\prime}\right)$, temos que $\tilde{p}^{\prime}$ pertence a $R\left(\tilde{\gamma}_{1}^{\prime}\right) \cap L\left(\tilde{\gamma}_{2}^{\prime}\right)$ e portanto, pelo Lema 4.15 , pertence a uma componente conexa limitada de $R\left(\tilde{\gamma}_{1}^{\prime}\right) \cap L\left(\tilde{\gamma}_{2}^{\prime}\right)$. Mas a componente conexa $C$ de $r\left(\tilde{\gamma}_{1}^{\prime}\right)$ que contém $\tilde{p}^{\prime}$ é ilimitada, pelo Lema 4.9, e portanto precisa intersectar $\left[\tilde{\gamma}_{1}^{\prime}\right] \cup\left[\tilde{\gamma}_{2}^{\prime}\right]$. Como $C$ é disjunta de $\left[\tilde{\gamma}_{1}^{\prime}\right]$, temos que a mesma intersecta $\left[\tilde{\gamma}_{2}^{\prime}\right]$, o que implica que existe $\bar{t}$ tal que $\left[\phi_{\tilde{\gamma}_{2}^{\prime}(\bar{t})}\right] \subset r\left(\tilde{\gamma}_{0}\right)$ e como $\tilde{\gamma}_{2}^{\prime} \sim_{\tilde{\mathcal{F}}} \tilde{\gamma}_{0}+w$, isso implica que algum ponto de $\tilde{\gamma}_{0}+w$ está na folha $\phi_{\tilde{\gamma}_{2}^{\prime}(\bar{t})}$, e portanto também está em $r\left(\tilde{\gamma}_{0}\right)$. A prova que $l\left(\tilde{\gamma}_{0}+w\right)$ intersecta $\tilde{\gamma}_{0}$ é análoga. 
Observação 4.17. Note que podemos obter um resultado simétrico ao anterior, com $r\left(\tilde{\gamma}_{0}+w\right) \cap$ $l\left(\tilde{\gamma}_{0}\right) \neq \emptyset$.

Seja $p$ uma singularidade de $\mathcal{F}$ e fixemos $\tilde{p}$ um levantamento de $p$. Temos que $\tilde{p}$ é fixo por $\tilde{f}$ e suponhamos que $\tilde{p} \in l\left(\tilde{\gamma}_{0}\right)$.

Lema 4.18. Sejam $w, w^{\prime} \in \mathbb{Z}^{2}$ tais que $\left\langle w, \rho_{0}^{\perp}\right\rangle>0$ e $\tilde{p}+w^{\prime} \in l\left(\tilde{\gamma}_{0}\right)$, ent $\tilde{a} o\left(\tilde{p}+w^{\prime}\right)+w \in l\left(\tilde{\gamma}_{0}\right)$.

Demonstração. Suponhamos, por absurdo, que $\left(\tilde{p}+w^{\prime}\right)+w \in r\left(\tilde{\gamma}_{0}\right)$. Note que $\left(\tilde{p}+w^{\prime}\right)+w \in l\left(\tilde{\gamma}_{0}+w\right)$, portanto temos que $l\left(\tilde{\gamma}_{0}+w\right) \cap r\left(\tilde{\gamma}_{0}\right) \neq \emptyset$ (veja a Figura 4.9). Assim, pelo Lema 4.16, temos que $\left[\tilde{\gamma}_{0}+w\right] \cap r\left(\tilde{\gamma}_{0}\right) \neq \emptyset$.

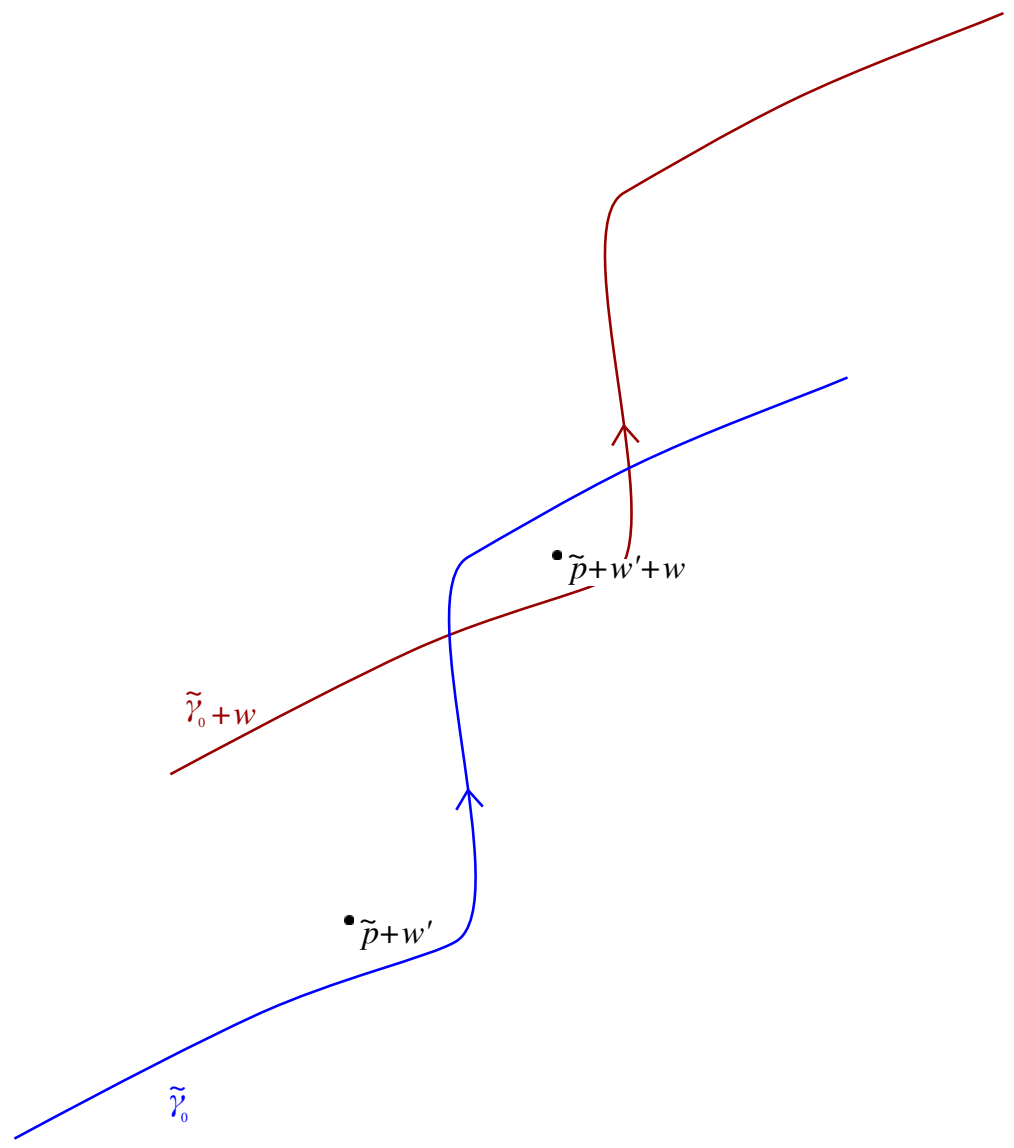

Figura 4.9: Ilustração do Lema 4.18

Afirmamos que isso implica que $r\left(\tilde{\gamma}_{0}+w\right) \subset \mathbb{R}^{2} \backslash l\left(\tilde{\gamma}_{0}\right)$ : de fato, se $r\left(\tilde{\gamma}_{0}+w\right) \cap l\left(\tilde{\gamma}_{0}\right) \neq \emptyset$, temos novamente pelo Lema 4.16 que $\left[\tilde{\gamma}_{0}+w\right] \cap l\left(\tilde{\gamma}_{0}\right) \neq \emptyset$. Obtemos assim pela Proposição 3.22 que $\tilde{\gamma}_{0} \pitchfork_{\mathcal{F}}\left(\tilde{\gamma}_{0}+w\right)$, o que é um absurdo pelo Lema 4.6, provando a afirmação. Assim, como $\left(\tilde{p}+w^{\prime}\right)+2 w \in r\left(\tilde{\gamma}_{0}+w\right)$ e $r\left(\tilde{\gamma}_{0}+w\right) \subset \mathbb{R}^{2} \backslash l\left(\tilde{\gamma}_{0}\right)$, obtemos $\left(\tilde{p}+w^{\prime}\right)+2 w \in r\left(\tilde{\gamma}_{0}\right)$. Portanto, por indução, temos que $\left(\tilde{p}+w^{\prime}\right)+n w \in r\left(\tilde{\gamma}_{0}\right)$, para todo $n \in \mathbb{N}$.

Denotemos agora $v_{w}^{\prime}=w /\|w\|$. Sendo $\theta$ o menor ângulo entre $v_{w}^{\prime}$ e a reta gerada por $\rho_{0}$, seja agora $v_{w}$ um vetor unitário tal que seu ângulo com a reta gerada por $\rho_{0}$ é $\theta / 2$. Assim, temos que para $n$ suficientemente grande, $\left(\tilde{p}+w^{\prime}\right)+n w$ está na componente conexa de $\mathbb{R}^{2} \backslash\left(B\left(L_{0}, C_{\tilde{z}_{0}}^{\rho_{0}}\left(v_{w}\right)\right) \cup\right.$ $\left.B\left(L_{1}, \tilde{z}_{0}\right)\right)$ contida na esquerda da reta gerada por $\rho_{0}$ que passa por $\tilde{z}_{0}$ (veja a Figura 4.10). Mas, pelo Lema 4.4 , temos que $\left[\tilde{\gamma}_{0}\right] \subset B\left(L_{0}, C_{\tilde{z}_{0}}^{\rho_{0}}\left(v_{w}\right)\right) \cup B\left(L_{1}, \tilde{z}_{0}\right)$, portanto $\left(\tilde{p}+w^{\prime}\right)+n w \in l\left(\tilde{\gamma}_{0}\right)$, o que é uma contradição.

Note que com uma demonstração análoga podemos obter o seguinte:

Lema 4.19. Sejam $w, w^{\prime} \in \mathbb{Z}^{2}$ tais que $\left\langle w, \rho_{0}^{\perp}\right\rangle<0$ e $\tilde{p}+w^{\prime} \in r\left(\tilde{\gamma}_{0}\right)$, então $\left(\tilde{p}+w^{\prime}\right)+w \in r\left(\tilde{\gamma}_{0}\right)$. 


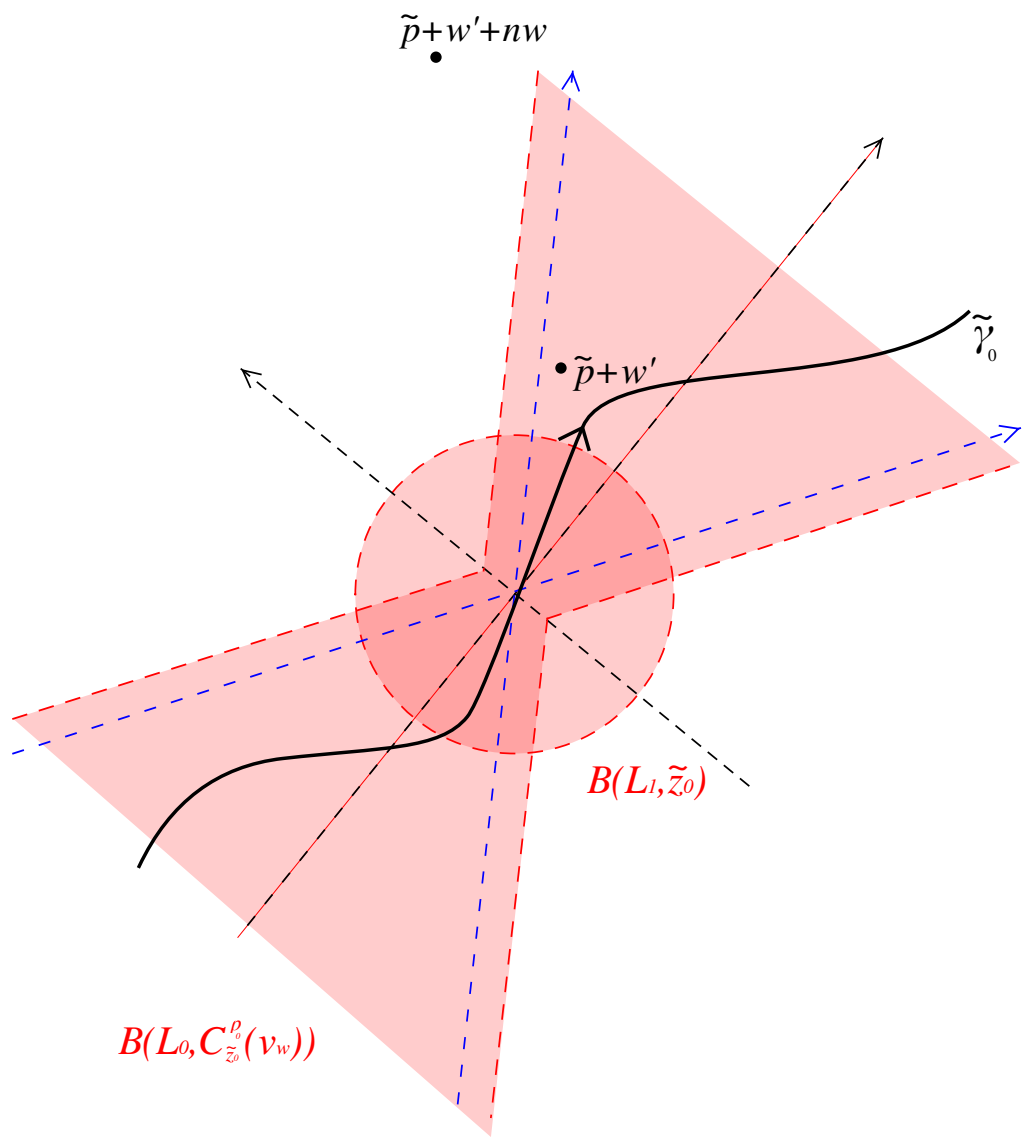

Figura 4.10: Ilustração do Lema 4.18

Como a inclinação de $\rho_{0}^{\perp}$ é irracional, podemos definir a seguinte ordem em $\mathbb{Z}^{2}$ :

Definição 4.20. $w \succ w^{\prime} \Leftrightarrow\left\langle w-w^{\prime}, \rho_{0}^{\perp}\right\rangle>0$.

Observação 4.21. Note que tal ordem é definida simplesmente projetando $\mathbb{Z}^{2}$ na reta gerada por $\rho_{0}^{\perp}$ e usando a ordem natural de tal reta. Além disso, temos que tal projeção é densa na reta.

Denotaremos por $Z_{r}=\left\{w \in \mathbb{Z}^{2} \mid \tilde{p}+w \in r\left(\tilde{\gamma}_{0}\right)\right\}$ e $Z_{l}=\left\{w \in \mathbb{Z}^{2} \mid \tilde{p}+w \in l\left(\tilde{\gamma}_{0}\right)\right\}$. Note que, como $\left(\tilde{p}+\mathbb{Z}^{2}\right) \cap\left[\tilde{\gamma}_{0}\right]=\emptyset$, temos $\mathbb{Z}^{2}=Z_{l} \cup Z_{r}$. O lema seguinte mostrará que as projeções de $Z_{r}$ e $Z_{l}$ na reta gerada por $\rho_{0}^{\perp}$ estão contidas em duas semirretas disjuntas.

Lema 4.22. Os conjuntos $Z_{r}$ e $Z_{l}$ definidos acima satisfazem as seguintes propriedades:

(i) Se $w \in Z_{l}$ e $w^{\prime} \succ w$, então $w^{\prime} \in Z_{l}$;

(ii) Se $w \in Z_{r}$ e $w^{\prime} \prec w$, então $w^{\prime} \in Z_{r}$.

Demonstração. Para provar $(i)$, tomemos $w \in Z_{l}$ e $w^{\prime} \succ w$. Temos assim que $\left\langle w^{\prime}-w, \rho_{0}^{\perp}\right\rangle>0$. Como $w \in Z_{l}$, temos por definição que $\tilde{p}+w \in l\left(\tilde{\gamma}_{0}\right)$ e assim, pelo Lema 4.18 , temos que $\tilde{p}+w+\left(w^{\prime}-w\right) \in$ $l\left(\tilde{\gamma}_{0}\right)$, e portanto $w^{\prime} \in Z_{l}$.

A prova de $(i i)$ é análoga.

Lema 4.23. Seja $w \in \mathbb{Z}^{2}$ tal que $w \prec 0$. Existe $w^{*} \in \mathbb{Z}^{2}$ tal que $w^{*} \in Z_{l}$ e $w^{*}+w \in Z_{r}$.

Demonstração. Denotemos $\left\langle A, \rho_{0}^{\perp}\right\rangle=\left\{\left\langle w, \rho_{0}^{\perp}\right\rangle \mid w \in A\right\}$. Usando tal notação, temos que $\left\langle\mathbb{Z}^{2}, \rho_{0}^{\perp}\right\rangle=$ $\left\langle Z_{l}, \rho_{0}^{\perp}\right\rangle \cup\left\langle Z_{r}, \rho_{0}^{\perp}\right\rangle$ e $\left\langle\mathbb{Z}^{2}, \rho_{0}^{\perp}\right\rangle$ é denso em $\mathbb{R}$. Vamos provar que $\left\langle Z_{l}, \rho_{0}^{\perp}\right\rangle$ é limitado inferiormente. Por absurdo, se $\left\langle Z_{l}, \rho_{0}^{\perp}\right\rangle$ for ilimitado inferiormente, temos que existem $w_{n} \in \mathbb{Z}^{2}, n \in \mathbb{N}$, tais que $\left\langle w_{n}, \rho_{0}^{\perp}\right\rangle \rightarrow-\infty$. Mas, pelo Lema 4.22 , se $w_{n} \in Z_{l}$, temos que $w^{\prime} \in Z_{l}$, se $w^{\prime} \succ w_{n}$, e portanto 
temos que $\left\langle Z_{l}, \rho_{0}^{\perp}\right\rangle=\left\langle\mathbb{Z}^{2}, \rho_{0}^{\perp}\right\rangle$, o que é um absurdo. Portanto $\left\langle Z_{l}, \rho_{0}^{\perp}\right\rangle$ é limitado inferiormente. Analogamente, temos que $\left\langle Z_{r}, \rho_{0}^{\perp}\right\rangle$ é limitado superiormente. Denotemos $l^{*}=\inf \left\langle Z_{l}, \rho_{0}^{\perp}\right\rangle$ e $r^{*}=$ $\sup \left\langle Z_{r}, \rho_{0}^{\perp}\right\rangle$. Temos que $l^{*}=r^{*}$. De fato, se $r^{*}<l^{*}$, temos um absurdo, pois teríamos $\left(r^{*}, l^{*}\right) \cap$ $\left\langle\mathbb{Z}^{2}, \rho_{0}^{\perp}\right\rangle=\emptyset$, e $\left\langle\mathbb{Z}^{2}, \rho_{0}^{\perp}\right\rangle$ é denso em $\mathbb{R}$. Se $l^{*}<r^{*}$, temos que existem $w_{l} \in Z_{l}$ e $w_{r} \in Z_{r}$ tais que $w_{r}-w_{l} \succ 0$. Portanto temos, pelo Lema 4.18 que $w_{l}+\left(w_{r}-w_{l}\right) \in l\left(\tilde{\gamma}_{0}\right)$, o que é um absurdo. Assim, $l^{*}=r^{*}$. Como $l^{*}=\inf \left\langle Z_{l}, \rho_{0}^{\perp}\right\rangle$, temos que existe uma sequência $w_{n} \in Z_{l}$ tal que $\left\langle w_{n}, \rho_{0}^{\perp}\right\rangle \rightarrow l^{*}$. Assim, como $\left\langle w, \rho_{0}^{\perp}\right\rangle<0$, temos que existe $n^{\prime} \in \mathbb{N}$ tal que $\left\langle w_{n^{\prime}}+w, \rho_{0}^{\perp}\right\rangle<l^{*}$. Portanto, tomando $w^{*}=w_{n^{\prime}}$, temos que $w^{*} \in Z_{l}$ e $w^{*}+w \in Z_{r}$, provando o lema.

Observação 4.24. De forma análoga ao Lema anterior, dado $w \succ 0$, podemos obter $w^{* *} \in \mathbb{Z}^{2}$ tal que $w^{* *} \in Z_{r}$ e $w^{* *}+w \in Z_{l}$

Lema 4.25. Seja $w \in \mathbb{Z}_{*}^{2}$. Se $w \succ 0$, $\tilde{\gamma}_{0}$ intersecta uma folha em $r\left(\tilde{\gamma}_{0}+w\right)$ e $\tilde{\gamma}_{0}+w$ intersecta uma folha em $l\left(\tilde{\gamma}_{0}\right)$. Se $w \prec 0, \tilde{\gamma}_{0}$ intersecta uma folha em $l\left(\tilde{\gamma}_{0}+w\right)$ e $\tilde{\gamma}_{0}+w$ intersecta uma folha em $r\left(\tilde{\gamma}_{0}\right)$. Em particular temos que $\tilde{\gamma}_{0}$ e $\tilde{\gamma}_{0}+w$ não são $\tilde{\mathcal{F}}$-equivalentes.

Demonstração. Vejamos o caso em que $w \prec 0$. Pelo Lema 4.23 , temos que existe $w^{*} \in \mathbb{Z}^{2}$ tal que $\tilde{p}+w^{*} \in l\left(\tilde{\gamma}_{0}\right)$ e $\tilde{p}+w^{*}+w \in r\left(\tilde{\gamma}_{0}\right)$, e assim $\tilde{p}+w^{*}+w \in r\left(\tilde{\gamma}_{0}\right) \cap l\left(\tilde{\gamma}_{0}+w\right)$. Pelo Lema 4.16, temos que $\left[\tilde{\gamma}_{0}\right] \cap l\left(\tilde{\gamma}_{0}+w\right) \neq \emptyset$ e $\left[\tilde{\gamma}_{0}+w\right] \cap r\left(\tilde{\gamma}_{0}\right) \neq \emptyset$, provando o resultado.

A prova para $w \succ 0$ é análoga, usando a Observação 4.24.

Lema 4.26. Dado $t_{1}>0$, existe $0<\varepsilon<\frac{1}{2}$ tal que, se $\tilde{z}^{\prime} \in B\left(\varepsilon, \tilde{z}_{0}\right)$, então todo representante de $\tilde{I}_{\tilde{\mathcal{F}}}^{\mathbb{Z}}\left(\tilde{z}^{\prime}\right)$ contém um subcaminho $\tilde{\mathcal{F}}$-equivalente a $\left.\tilde{\gamma}_{0}\right|_{\left[0, t_{1}\right]}$.

Demonstração. Segue diretamente pelo Lema 3.23.

No que se segue, diremos que $f$ não possui desvio limitado na direção positiva de $\rho_{0}^{\perp}$ se existem sequências $\tilde{x}_{k} \in \mathbb{R}^{2}$ e $\left(n_{k}\right)_{k \in \mathbb{N}}$ crescente tais que $\lim _{k \rightarrow \infty}\left\langle\tilde{f}^{n_{k}}\left(\tilde{x}_{k}\right)-\tilde{x}_{k}, \rho_{0}^{\perp}\right\rangle=+\infty$. Analogamente, diremos que $f$ não possui desvio limitado na direção negativa de $\rho_{0}^{\perp}$ se existirem $\tilde{x}$ e $\left(n_{k}\right)_{k \in \mathbb{N}}$ como antes tais que o limite anterior for igual a $-\infty$. Note que se $f$ não possui desvio limitado na direção de $\rho_{0}^{\perp}$ então $f$ não possui desvio limitado na direção positiva de $\rho_{0}^{\perp}$ ou $f$ não possui desvio limitado na direção negativa de $\rho_{0}^{\perp}$.

Lema 4.27. Se $f$ não possui desvio limitado na direção positiva de $\rho_{0}^{\perp}$, então, dado $0<\varepsilon<\frac{1}{2}$, existem $\tilde{x} \in \mathbb{R}^{2}, N \in \mathbb{N}$ e $P \in \mathbb{Z}^{2}$ tais que:

(i) $\tilde{x} \in B\left(\varepsilon, \tilde{z}_{0}\right)$

(ii) $P \succ(-2,0)$

(iii) $\tilde{f}^{N}(\tilde{x}) \in B\left(\varepsilon, \tilde{z}_{0}+P\right)$

Demonstração. Temos pelo Lema 2.25 que o conjunto $U_{\varepsilon}=\bigcup_{i=0}^{\infty} f^{i}\left(\pi\left(B\left(\varepsilon, \tilde{z}_{0}\right)\right)\right)$ é totalmente essencial, e como $\varepsilon<\frac{1}{2}$, temos que $\overline{\pi\left(B\left(\varepsilon, \tilde{z}_{0}\right)\right)}$ é inessencial. Assim, aplicando a Proposição 2.24, obtemos um compacto do plano $K$ e $M \in \mathbb{N}$ tal que $[0,1]^{2}$ está contido numa componente conexa

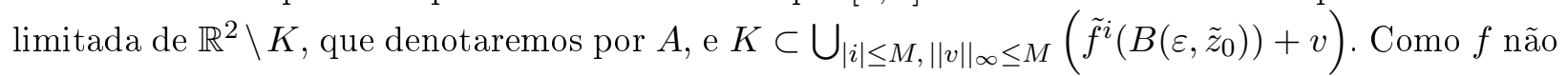
possui desvio limitado na direção positiva de $\rho_{0}^{\perp}$, existem $P^{\prime} \in \mathbb{Z}^{2}$ e $l \in \mathbb{N}$ tais que $l>2 M,\left\langle P^{\prime}, \rho_{0}^{\perp}\right\rangle>$ $\left\langle-(2,0), \rho_{0}^{\perp}\right\rangle+2 M$ e $\tilde{f}^{l}\left([0,1]^{2}\right) \cap\left([0,1]^{2}+P^{\prime}\right) \neq \emptyset$. Como $[0,1]^{2} \subset A$ e $\tilde{f}^{l}\left([0,1]^{2}\right) \cap\left([0,1]^{2}+P^{\prime}\right) \neq \emptyset$, temos que $\tilde{f}^{l}(A)$ intersecta $A+P^{\prime}$. Como $A$ é limitado, temos que $\tilde{f}^{l}(\partial A) \cap\left(\partial A+P^{\prime}\right) \neq \emptyset$, e como $\partial A \subset K$, obtemos $\tilde{f}^{l}(K) \cap\left(K+P^{\prime}\right) \neq \emptyset$. Seja agora $\tilde{y} \in \tilde{f}^{l}(K) \cap\left(K+P^{\prime}\right)$. Então existem $n_{i} \in \mathbb{Z}$, $\left|n_{i}\right|<M$ e $v_{i} \in \mathbb{Z}^{2},\left\|v_{i}\right\|_{\infty}<M$, para $i=1,2$, tais que

$$
\begin{aligned}
\tilde{y} \in \tilde{f}^{l}(K) & \Rightarrow \tilde{y} \in \tilde{f}^{l}\left(\tilde{f}^{n_{1}}\left(B\left(\varepsilon, \tilde{z}_{0}\right)\right)+v_{1}\right)=\tilde{f}^{l+n_{1}}\left(B\left(\varepsilon, \tilde{z}_{0}\right)\right)+v_{1} \\
\tilde{y} \in K+P^{\prime} & \Rightarrow \tilde{y} \in \tilde{f}^{n_{2}}\left(B\left(\varepsilon, \tilde{z}_{0}\right)\right)+v_{2}+P^{\prime} .
\end{aligned}
$$


Assim obtemos

$$
\begin{aligned}
\tilde{f}^{-n_{2}}(\tilde{y}) & \in\left(\tilde{f}^{l+n_{1}-n_{2}}\left(B\left(\varepsilon, \tilde{z}_{0}\right)\right)+v_{1}\right) \cap B\left(\varepsilon, \tilde{z}_{0}+v_{2}+P^{\prime}\right) \\
\tilde{f}^{-n_{2}}(\tilde{y})-v_{1} & \in \tilde{f}^{l+n_{1}-n_{2}}\left(B\left(\varepsilon, \tilde{z}_{0}\right)\right) \cap B\left(\varepsilon, \tilde{z}_{0}+v_{2}-v_{1}+P^{\prime}\right) .
\end{aligned}
$$

Assim, fazendo $N=l+n_{1}-n_{2}$ e $P=v_{2}-v_{1}+P^{\prime}$, obtemos o resultado.

Note que podemos provar um resultado análogo para o caso em que $f$ não possui desvio limitado na direção negativa de $\rho_{0}^{\perp}$.

Com todos os resultados provados até agora podemos finalizar a demonstração do Teorema A.

Demonstração do Teorema A. Suponhamos por absurdo que $f$ não possui desvio limitado na direção de $\rho_{0}^{\perp}$. Vamos assumir que $f$ não possui desvio limitado na direção positiva de $\rho_{0}^{\perp}$ (o outro caso é análogo). Vamos relembrar também que assumimos que $\rho_{0}$ está no primeiro quadrante.

Como $(1,0) \prec 0$, temos pelo Lema 4.25 que $\tilde{\gamma}_{0}$ e $\tilde{\gamma}_{0}+(1,0)$ não são $\tilde{\mathcal{F}}$-equivalentes, e além disso $\tilde{\gamma}_{0}$ intersecta uma folha em $l\left(\tilde{\gamma}_{0}+(1,0)\right)$. Seja $t_{0}$ um instante em que tal intersecção ocorre, ou seja, $\tilde{\gamma}_{0}\left(t_{0}\right)$ está em uma folha, que denotaremos por $\phi_{l}$, que está contida em $l\left(\tilde{\gamma}_{0}+(1,0)\right)$. Analogamente, como $-(1,0) \succ 0$ temos pelo Lema 4.25 que $\tilde{\gamma}_{0}$ e $\tilde{\gamma}_{0}-(1,0)$ não são $\tilde{\mathcal{F}}$-equivalentes, e além disso $\tilde{\gamma}_{0}$ intersecta uma folha em $r\left(\tilde{\gamma}_{0}-(1,0)\right)$. Seja $t_{1}$ tal que $\tilde{\gamma}_{0}\left(t_{1}\right)$ pertence a uma folha, que denotaremos por $\phi_{r}$, que está contida em $r\left(\tilde{\gamma}_{0}-(1,0)\right)$. Note que se $t_{0}$ ou $t_{1}$ for menor que 0 , podemos substituir $\tilde{z_{0}}$ por $\tilde{z}_{0}^{\prime}=\tilde{f}^{-n}\left(\tilde{z_{0}}\right)$ para $n$ suficientemente grande e assim reparametrizar $\tilde{\gamma}_{0}$. Podemos assumir, sem perda de generalidade, que $0<t_{0}<t_{1}$. A posição relativa entre $t_{0}$ e $t_{1}$ não terá influência no restante da demonstração, mas tal suposição simplificará algumas notações. Seja agora $0<\varepsilon<\frac{1}{2}$ dado pelo Lema 4.26, e sejam também $\tilde{x} \in \mathbb{R}^{2}, N \in \mathbb{N}$ e $P \in \mathbb{Z}^{2}$ dados pelo Lema 4.27. Denotaremos um representante de $\tilde{I}_{\tilde{\mathcal{F}}}^{Z}(\tilde{x})$ por $\beta_{\tilde{x}}$.

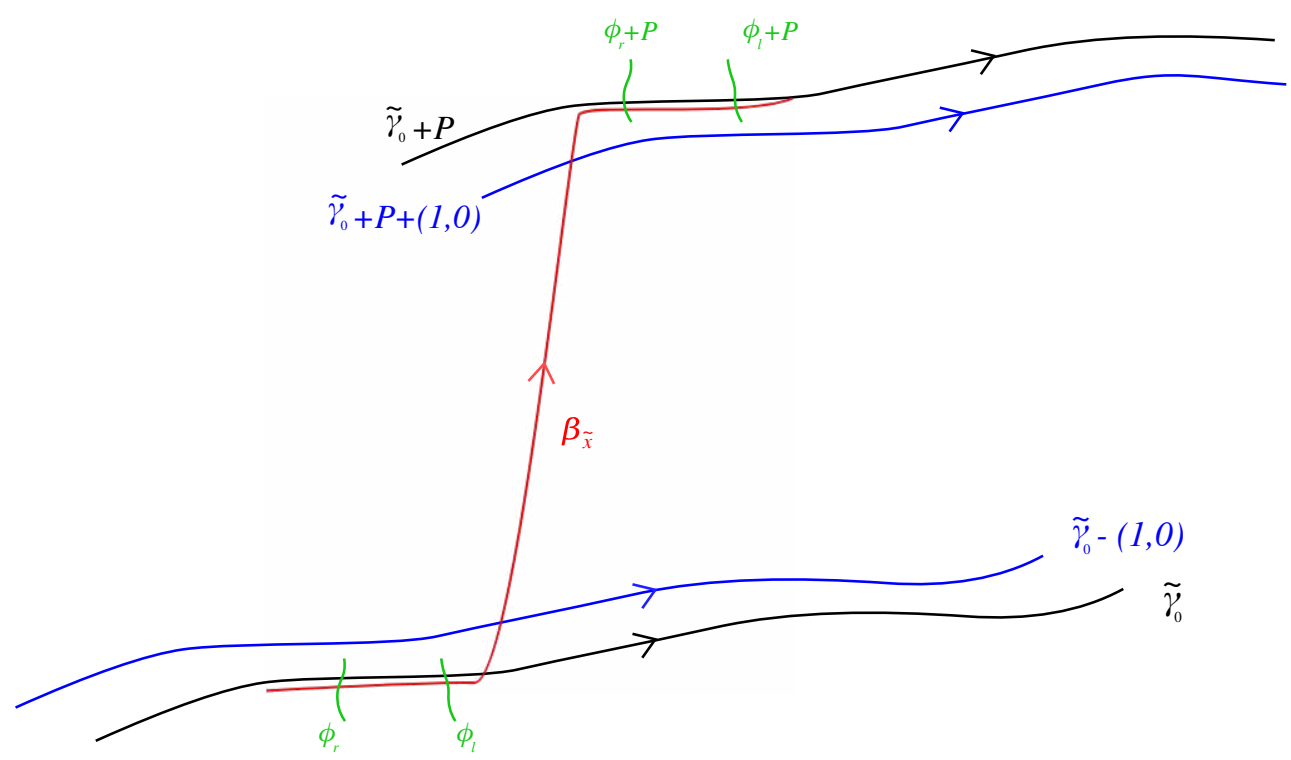

Figura 4.11: Construção de $\beta_{\tilde{x}}$

Vamos provar que $\beta_{\tilde{x}}$ intersecta $r\left(\tilde{\gamma}_{0}-(1,0)\right)$ e $l\left(\tilde{\gamma}_{0}+P+(1,0)\right)$. Como $\tilde{x} \in B\left(\varepsilon, \tilde{z}_{0}\right)$, temos pelo Lema 4.26 que $\left.\tilde{\gamma}_{0}\right|_{\left[0, t_{1}\right]}$ é equivalente a um subcaminho de $\beta_{\tilde{x}}$, assim temos que $\beta_{\tilde{x}}$ cruza $\phi_{r}$. Da mesma forma, como $\tilde{f}^{N}(\tilde{x}) \in B\left(\varepsilon, \tilde{z}_{0}+P\right)$, temos que $\left.\tilde{\gamma}_{0}\right|_{\left[0, t_{1}\right]}+P$ é equivalente a um subcaminho de $\beta_{\tilde{x}}$, e portanto $\beta_{\tilde{x}}$ intersecta $\phi_{l}+P$. Denotemos por $I=[a, b]$ um intervalo tal que $\left[\left.\beta_{\tilde{x}}\right|_{I}\right] \cap\left[\phi_{r}\right] \neq \emptyset$, $\left[\left.\beta_{\tilde{x}}\right|_{I}\right] \cap\left[\phi_{l}+P\right] \neq \emptyset$ e $\left.\beta_{\tilde{x}}\right|_{I}$ é admissível. 
Afirmamos que $\left.\left(\tilde{\gamma}_{0}+w\right) \pitchfork_{\tilde{\mathcal{F}}} \beta_{\tilde{x}}\right|_{I}$ se $w \in \mathbb{Z}^{2}$ é tal que $-(1,0) \prec w \prec P+(1,0)$. Vamos provar primeiramente que $\left[\phi_{l}+P\right] \subset l\left(\tilde{\gamma}_{0}+w\right)$ e $\left[\phi_{r}\right] \subset r\left(\tilde{\gamma}_{0}+w\right)$. Suponhamos por contradição que $\left[\phi_{r}\right] \not \subset$ $r\left(\tilde{\gamma}_{0}+w\right)$. Temos então duas possibilidades: $\left[\phi_{r}\right] \subset l\left(\tilde{\gamma}_{0}+w\right)$ ou $\tilde{\gamma}_{0}+w$ cruza $\phi_{r}$. Se $\left[\phi_{r}\right] \subset l\left(\tilde{\gamma}_{0}+w\right)$, temos que $\left[\tilde{\gamma}_{0}\right] \cap l\left(\tilde{\gamma}_{0}+w\right) \neq \emptyset$. Além disso,como $0 \prec-(1,0) \prec w$, temos pelo Lema 4.25 que $\left[\tilde{\gamma}_{0}\right] \cap r\left(\tilde{\gamma}_{0}+w\right) \neq \emptyset$. Portanto, pelo Corolário 4.11 , temos que $\tilde{\gamma}_{0} \pitchfork_{\tilde{\mathcal{F}}} \tilde{\gamma}_{0}+w$, o que é um absurdo, pelo Lema 4.5. Se $\tilde{\gamma}_{0}+w$ cruza $\phi_{r}$, como $\left[\phi_{r}\right] \subset r\left(\tilde{\gamma}_{0}-(1,0)\right)$, temos $\left[\tilde{\gamma}_{0}+w\right] \cap r\left(\tilde{\gamma}_{0}-(1,0)\right) \neq \emptyset$. Além disso, como $-(1,0) \prec w$, temos pelo Lema 4.25 que $\left[\tilde{\gamma}_{0}\right] \cap l\left(\tilde{\gamma}_{0}-(1,0)-w\right) \neq \emptyset$, e portanto $\left[\tilde{\gamma}_{0}+w\right] \cap l\left(\tilde{\gamma}_{0}-(1,0)\right) \neq \emptyset$. Novamente, pelo Corolário 4.11 temos que $\left(\tilde{\gamma}_{0}-(1,0)\right) \pitchfork_{\tilde{\mathcal{F}}}\left(\tilde{\gamma}_{0}+w\right)$, o que é um absurdo, pelo Lema 4.5. Portanto temos que $\left[\phi_{r}\right] \subset r\left(\tilde{\gamma}_{0}+w\right)$. De forma simétrica, usando o fato que $w \prec P+(1,0) \prec P$, podemos provar que $\left[\phi_{l}+P\right] \subset l\left(\tilde{\gamma}_{0}+w\right)$. Assim, pelo Corolário 4.11, temos que $\left.\left(\tilde{\gamma}_{0}+w\right) \pitchfork_{\tilde{\mathcal{F}}} \beta_{\tilde{x}}\right|_{I}$, provando a afirmação..

Agora sejam $w_{1}, w_{2} \in \mathbb{Z}^{2}$, tais que $-(1,0) \prec w_{i} \prec P+(1,0)$. Como $\left.\left(\tilde{\gamma}_{0}+w_{i}\right) \pitchfork_{\tilde{\mathcal{F}}} \beta_{\tilde{x}}\right|_{I}$, temos que existem $t^{\prime}, t^{\prime \prime}, s^{\prime}, s^{\prime \prime} \in \mathbb{R}$ tais que $\left(\tilde{\gamma}_{0}+w_{1}\right)\left(t^{\prime}\right)=\beta_{\tilde{x}}\left(s^{\prime}\right)$ e $\left(\tilde{\gamma}_{0}+w_{2}\right)\left(t^{\prime \prime}\right)=\beta_{\tilde{x}}\left(s^{\prime \prime}\right)$, e podemos assumir que $s^{\prime}<s^{\prime \prime}$. Seja ainda $J \subset \mathbb{R}$ um intervalo fechado tal que $t^{\prime \prime} \in J,\left.\left.\left(\tilde{\gamma}_{0}+w_{2}\right)\right|_{J} \pitchfork_{\tilde{\mathcal{F}}} \beta_{\tilde{x}}\right|_{I}$ e $\left.\left(\tilde{\gamma}_{0}+w_{2}\right)\right|_{J}$ é admissível. Como $z_{0}$ é recorrente, temos pelo Corolário 3.25 que $\pi\left(\tilde{\gamma}_{0}\right)$ é $\mathcal{F}$-recorrente, portanto existe um intervalo fechado $J^{\prime} \subset\left(-\infty, t^{\prime}\right]$ e $w_{3} \in \mathbb{Z}^{2}$ tais que $\left.\left.\left(\tilde{\gamma}_{0}+w_{2}\right)\right|_{J} \sim_{\tilde{\mathcal{F}}}\left(\tilde{\gamma}_{0}+w_{3}\right)\right|_{J^{\prime}}$. Note que podemos assumir que $w_{3} \neq w_{1}$, bastando substituir $t^{\prime}$ por $\bar{t}$ suficientemente distante de $t^{\prime}$, se necessário. Portanto temos que $\left.\left(\tilde{\gamma}_{0}+w_{3}\right)\right|_{J^{\prime}}$ é admissível e $\left.\left.\left(\tilde{\gamma}_{0}+w_{3}\right)\right|_{J^{\prime}} \pitchfork_{\tilde{\mathcal{F}}} \beta_{\tilde{x}}\right|_{I}$. Seja agora $[c, d] \subset \mathbb{R}$ tal que $t^{\prime} \in(c, d), J^{\prime} \subset[c, d]$ e $\left.\left(\tilde{\gamma}_{0}+w_{1}\right)\right|_{[c, d]}$ é admissível. Assim, pela Proposição 3.19, temos que $\beta^{\prime}=\left.\left.\left(\tilde{\gamma}_{0}+w_{1}\right)\right|_{\left[c, t^{\prime}\right]} \beta_{\tilde{x}}\right|_{\left[s^{\prime}, b\right]}$ é admissível, e como $s^{\prime}<s^{\prime \prime}$, temos que $\left.\left(\tilde{\gamma}_{0}+w_{3}\right)\right|_{J^{\prime}} \pitchfork_{\tilde{\mathcal{F}}} \beta^{\prime}$. Mas como

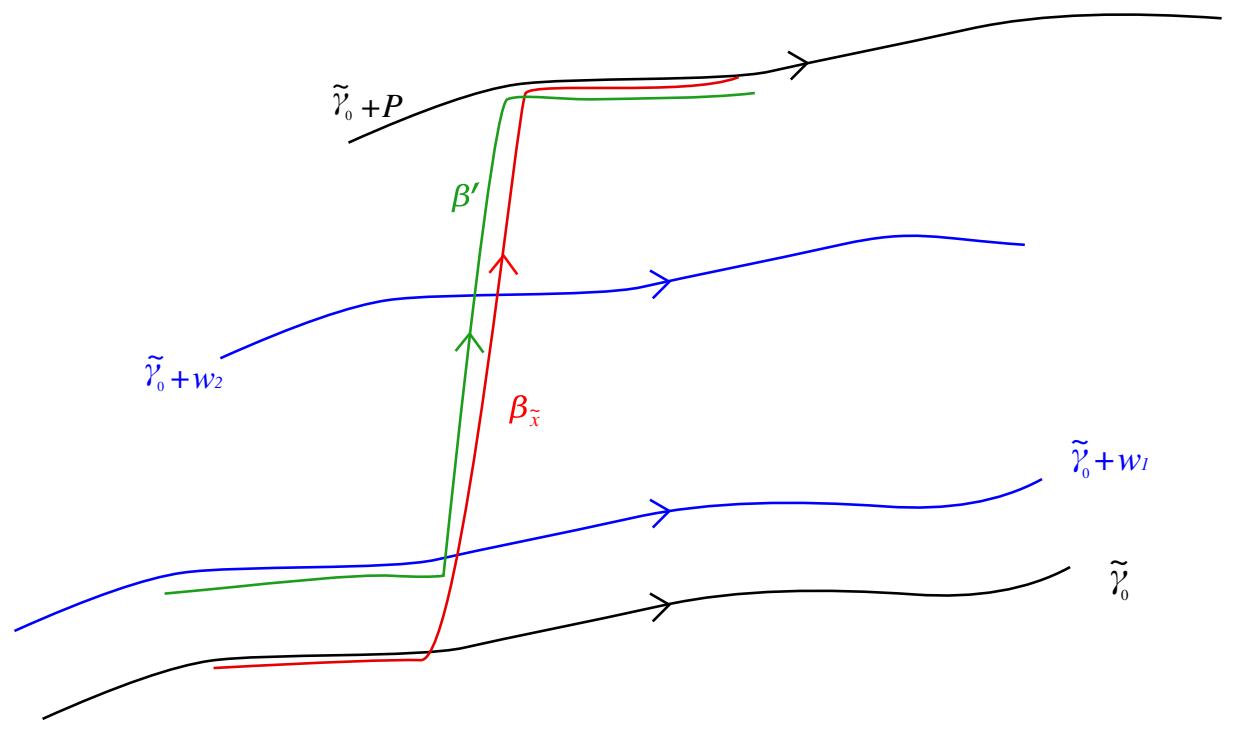

Figura 4.12: Construção de $\beta^{\prime}$

$J^{\prime} \subset\left[c, t^{\prime}\right]$, temos que $\beta^{\prime} \pitchfork_{\tilde{\mathcal{F}}}\left(\beta^{\prime}+w_{3}-w_{1}\right)$, o que é um absurdo, pelo Lema 4.5 , terminando assim a demonstração. 


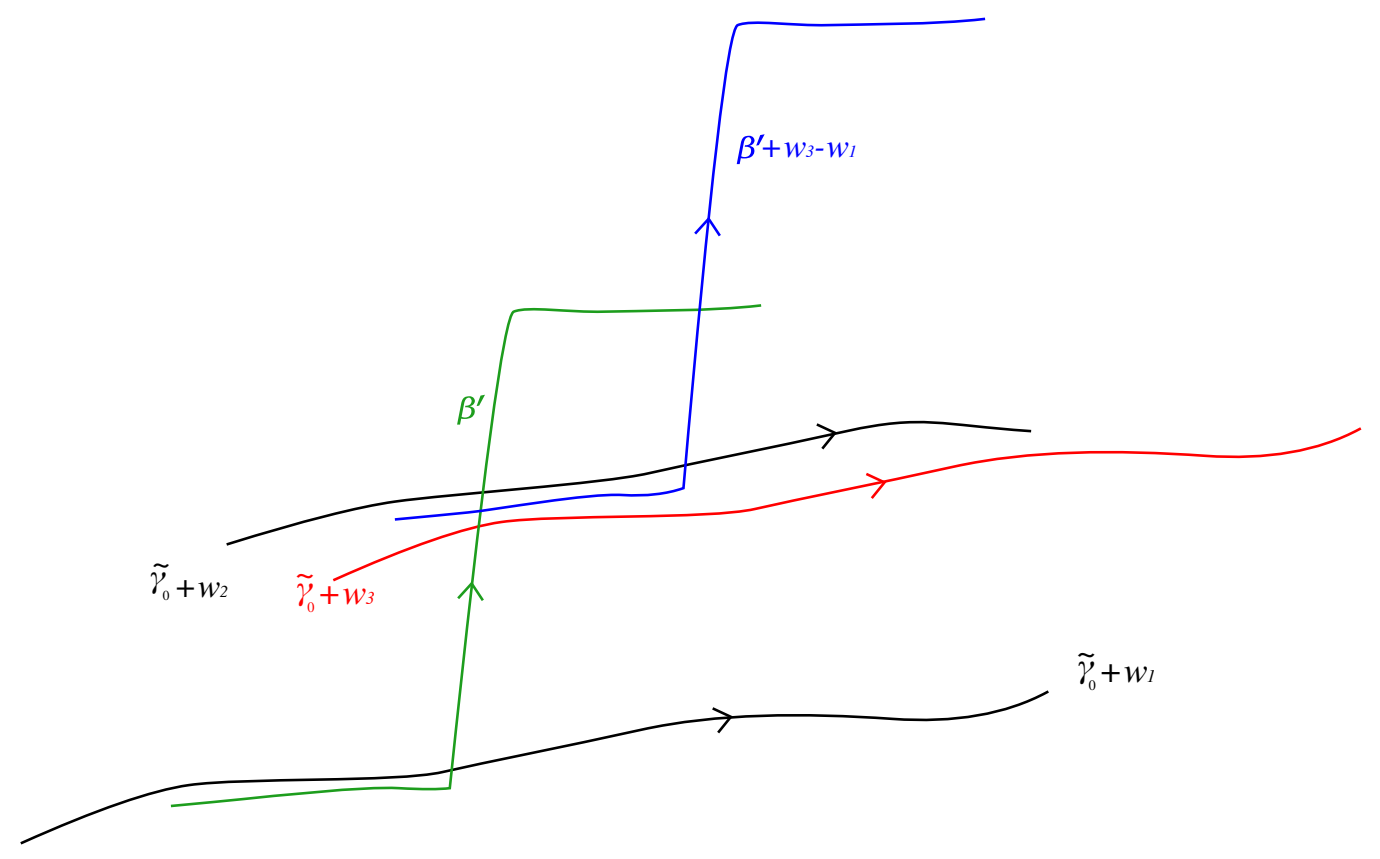

Figura 4.13: Intersecção de $\beta^{\prime} \operatorname{com} \beta^{\prime}+w_{3}-w_{1}$ 


\section{Referências Bibliográficas}

[AZ15] Salvador Addas-Zanata. Uniform bounds for diffeomorphisms of the torus and a conjecture of boyland. Journal of the London Mathematical Society, 91(2):537-553, 2015. 8

[AZTG12] Salvador Addas-Zanata, Fabio Armando Tal e Bráulio A Garcia. Dynamics of homeomorphisms of the torus homotopic to dehn twists. Ergodic Theory and Dynamical Systems, 34(2):409-422, 2012. 9

[BCR16] François Béguin, Sylvain Crovisier e Frédéric Le Roux. Fixed point sets of isotopies on surfaces. arXiv preprint arXiv:1610.00686, 2016. 17

[BdCTH16] Philip Boyland, André de Carvalho Toby e Hall. New rotation sets in a family of torus homeomorphisms. Inventiones mathematicae, 204(3):895-937, 2016. 5

[Bro12] Luitzen Egbertus Jan Brouwer. Beweis des ebenen translationssatzes. Mathematische Annalen, 72(1):37-54, 1912. 13

[Cal05] Patrice Le Calvez. Une version feuilletée équivariante du théoreme de translation de brouwer. Publications Mathématiques de l'Institut des Hautes Études Scientifiques, 102(1):1-98, 2005. 14, 18

[CT] Patrice Le Calvez e Fabio Armando Tal. Errata de "forcing theory for transverse trajectories of surface homeomorphisms". A ser publicado. 18

[CT18a] Patrice Le Calvez e Fabio Armando Tal. Forcing theory for transverse trajectories of surface homeomorphisms. Inventiones mathematicae, 212(2):619-729, 2018. 5, 6, 7, 8, $14,15,17,18,19$

[CT18b] Patrice Le Calvez e Fabio Armando Tal. Topological horseshoes for surface homeomorphisms. arXiv preprint arXiv:1803.04557, 2018. 19, 27, 28

[Dáv16] Pablo Dávalos. On annular maps of the torus and sublinear diffusion. Journal of the Institute of Mathematics of Jussieu, páginas 1-66, 2016. 8, 9

[FM90] John Franks e Michał Misiurewicz. Rotation sets of toral flows. Proceedings of the American Mathematical Society, 109(1):243-249, 1990. 5

[Fra88] John Franks. Recurrence and fixed points of surface homeomorphisms. Ergodic theory and dynamical systems, $8\left(8^{*}\right): 99-107,1988.5,6$

[Fra89] John Franks. Realizing rotation vectors for torus homeomorphisms. Transactions of the American Mathematical Society, 311(1):107-115, 1989. 6

[Fra92] John Franks. A new proof of the brouwer plane translation theorem. Ergodic Theory and Dynamical Systems, 12(2):217-226, 1992. 13

[Fra95] John Franks. The rotation set and periodic points for torus homeomorphisms. Dynamical systems and chaos, 1:41-48, 1995. 6 
[GKT14] Nancy Guelman, Andres Koropecki e Fabio Armando Tal. A characterization of annularity for area-preserving toral homeomorphisms. Mathematische Zeitschrift, 276(34):673-689, 2014. 8

[GKT15] Nancy Guelman, Andres Koropecki e Fabio Armando Tal. Rotation sets with nonempty interior and transitivity in the universal covering. Ergodic Theory and Dynamical Systems, 35(3):883-894, 2015. 10

[Han89] Michael Handel. Periodic point free homeomorphism of t2. Proceedings of the American Mathematical Society, 107(2):511-515, 1989. 5

[Jäg09a] Tobias Jäger. The concept of bounded mean motion for toral homeomorphisms. Dynamical Systems, 24(3):277-297, 2009. 8

[Jäg09b] Tobias Jäger. Linearization of conservative toral homeomorphisms. Inventiones mathematicae, 176(3):601-616, 2009. 8

[Jau14] Olivier Jaulent. Existence d'un feuilletage positivement transverse à un homéomorphisme de surface. Ann. Inst. Fourier, 64(4):1441-1476, 2014. 17

[JT17] Tobias Jäger e Fabio Armando Tal. Irrational rotation factors for conservative torus homeomorphisms. Ergodic Theory and Dynamical Systems, 37(5):1537-1546, 2017. 9

[JZ98] Leo B Jonker e Lei Zhang. Torus homeomorphisms whose rotation sets have empty interior. Ergodic Theory and Dynamical Systems, 18(5):1173-1185, 1998. 6

[KH95] Anatole Katok e Boris Hasselblatt. Introduction to the modern theory of dynamical systems, volume 54. Cambridge university press, 1995. 3

[Koc16] Alejandro Kocsard. On the dynamics of minimal homeomorphisms of t2 which are not pseudo-rotations. arXiv preprint arXiv:1611.03784, 2016. 5, 9

[KPS16] Andres Koropecki, Alejandro Passeggi e Martín Sambarino. The franks-misiurewicz conjecture for extensions of irrational rotations. arXiv preprint arXiv:1611.05498, 2016. 5

[KR17] Alejandro Kocsard e Fernanda Pereira Rodrigues. Rotational deviations and invariant pseudo-foliations for periodic point free torus homeomorphisms. Mathematische Zeitschrift, páginas 1-25, 2017. 9

[KT14a] Andres Koropecki e Fabio Armando Tal. Area-preserving irrotational diffeomorphisms of the torus with sublinear diffusion. Proceedings of the American Mathematical Society, 142(10):3483-3490, 2014. 7

[KT14b] Andres Koropecki e Fabio Armando Tal. Bounded and unbounded behavior for areapreserving rational pseudo-rotations. Proceedings of the London Mathematical Society, 109(3):785-822, 2014. 7

[KT14c] Andres Koropecki e Fabio Armando Tal. Strictly toral dynamics. Inventiones mathematicae, 196(2):339-381, 2014. 9

[KT18] Andres Koropecki e Fabio Armando Tal. Fully essential dynamics for area-preserving surface homeomorphisms. Ergodic Theory and Dynamical Systems, 38(5):1791-1836, 2018. 9

[Kwa92] Jaroslaw Kwapisz. Every convex polygon with rational vertices is a rotation set. Ergodic Theory and Dynamical Systems, 12(2):333-339, 1992. 5 
[Kwa95] Jaroslaw Kwapisz. A toral diffeomorphism with a non-polygonal rotation set. Nonlinearity, 8(4):461-476, 1995. 5

[LM91] Jaume Llibre e Robert S MacKay. Rotation vectors and entropy for homeomorphisms of the torus isotopic to the identity. Ergodic Theory and Dynamical Systems, 11(1):115$128,1991.5,6$

[MZ89] Michał Misiurewicz e Krystyna Ziemian. Rotation sets for maps of tori. Journal of the London Mathematical Society, 2(3):490-506, 1989. 1, 3, 4, 5

[MZ91] Michał Misiurewicz e Krystyna Ziemian. Rotation sets and ergodic measures for torus homeomorphisms. Fundamenta Mathematicae, 137(1):45-52, 1991. 5

[Poi81] Henri Poincaré. Mémoire sur les courbes définies par une équation différentielle (i). Journal de mathématiques pures et appliquées, 7:375-422, 1881. 3

[PS18] Alejandro Passeggi e Martín Sambarino. Deviations in the franks-misiurewicz conjecture. arXiv preprint arXiv:1803.03294, 2018. 5

[Wa100] Peter Walters. An introduction to ergodic theory, volume 79. Springer Science \& Business Media, 2000. 4 